\title{
Higher-Order Derivative Correlations and the Alignment of Small-Scale Structures in Isotropic Numerical Turbulence
}

Robert M. Kerr

LIBRARY GOPY

December 1983

\author{
JAN 101984 \\ LANGLEY RESEARCH LENTER \\ LIBRARY, NASA \\ HAMPTON, VIRGINIA
}

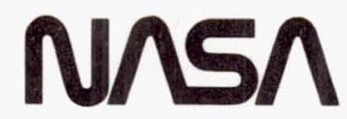




\section{Higher-Order Derivative Correlations and the Alignment of Small-Scale Structures in Isotropic Numerical Turbulence}

Robert M. Kerr, Ames Research Center, Moffett Field, California

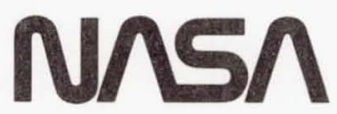

National Aeronautics and

Space Administration 


\title{
Higher-Order Derivative Correlations and the Alignment of Small-Scale Structures in Isotropic Numerical Turbulence
}

\author{
ROBERT M. KERR \\ NASA Ames Research Center \\ M.S. 202A-1, Moffett Field, CA 94035
}

August 1983

\begin{abstract}
In a three-dimensional simulation higher-order derivative correlations, including skewness and flatness (or kurtosis) factors, are calculated for velocity and passive scalar fields and are compared with structures in the flow. Up to $128^{3}$ grid points are used with periodic boundary conditions in all three directions to achieve $R_{\lambda}$ to 82.9 . The equations are forced to maintain steady-state turbulence and collect statistics. The scalarderivative flatness is found to increase much faster with Reynolds number than the velocity derivative flatness, and the velocity- and mixed-derivative skewnesses do not increase with Reynolds number. Separate exponents are found for the various fourth-order velocity-derivative correlations, with the vorticity-flatness exponent the largest. This does not support a major assumption of the lognormal and $\beta$ models, but is consistent with some aspects of structural models of the small scales. Three-dimensional graphics show strong alignment between the vorticity, rate-of-strain, and scalargradient fields. The vorticity is concentrated in tubes with the scalar gradient and the largest principal rate of strain aligned perpendicular to the tubes. Velocity spectra, in Kolmogorov variables, collapse to a single curve and a short $-5 / 3$ spectral regime is observed.
\end{abstract}

§A. Introduction The classical approach to investigating small-scale intermittency in turbulence is through the higher-order derivative correlations such as skewness and flatness factors. Experimentally this has been done for both the velocity and temperature, which is a passive scalar when buoyancy is negligible. The long-range goal is to improve our understanding of the structure of the small scales, possibly leading to improved methods for subgrid modelling. An intermediate objective has been to relate the derivative correlations to dissipation correlations and corrections to Kolmogorov scaling. It is believed that this is possible because the small scales are universal; that is, the small scales have a structure that is independent of the large scales and can be modeled. So far only single-probe velocity and scalar statistics, such as the velocity-derivative flatness and skewness, have been measured with hotwire instruments. More complicated statistics have not been measured and 
alternative approaches, such as flow visualization, are limited because they do not have the flexibility necessary to distinguish small-scale structures that are intermittent in space and time.

Another approach to investigating the small scales is numerical simulation. In a simulation more detail can be obtained than in experiments and the conditions can be more closely controlled. For example, because all components of the velocity are known, one is able to study correlations beyond the derivative skewness and flatness and computer graphics can display structures not accessible to experiments. The main disadvantage of a simulation is that only a limited range of length scales is allowed, which restricts the Reynolds number to very low values. But in low-Reynolds-number experiments the values of most of the derivative correlations are significantly different from their uncorrelated or Gaussian values. Therefore, these statistics are accessible to current numerical methods and computers. Siggia (1981a) used a numerical simulation to calculate higher-order correlations of the velocity, and discussed the relation between small-scale vortex structures and intermittency. Our approach will be to use a similiar simulation to look in more detail at the velocity-derivative statistics and the statistics of a passive scalar. Graphical display of small-scale vorticity, rate-of-strain, and scalar-gradient structures is used to interpret these statistics and comparisons with phenomenological theories and experiments are made.

There are two phenomenological approaches to predicting the small-scale statistics: either by assuming a form for vortical structures or a form for the energy cascade from large to small scales. Two models that are based on the cascade of energy are the lognormal theory of Kolmogorov (1962) and the $\beta$-model of Frisch, Sulem, and Nelkin (1979). Both theories predict a correction to the $k^{-5 / 3}$ inertial-range kinetic-energy spectrum of Kolmogorov (1941). They also predict that as the Reynolds number grows that the velocity fluctuations become increasingly localized, or intermittent, distributions become highly non-Gaussian, and the higher-order correlations, such as the derivative skewness and flatness factors, increase with Reynolds number with a power-law dependence. The power-law exponents depend on the details of each model and on $\mu$, the characteristic exponent of the dissipationdissipation correlation function in the inertial subrange, $\eta<<r<<L$,

$$
<\epsilon(x) \epsilon(x+r)>=A \epsilon^{2}(L / r)^{\mu}
$$

(Monin and Yaglom 1975, p. 618), where $A$ is a constant and $\mathrm{L}$ is defined by (12). All correlations of a given order are predicted to have the same 
power-law dependence. The lognormal model predicts that

$$
<\epsilon^{m}(x) \epsilon^{n}(x+r)>\sim(L / r)^{\mu m n}
$$

and that

$$
F_{\partial u n}=\frac{\left\langle\left(\frac{\partial u_{1}}{\partial x_{1}}\right)^{n}\right\rangle}{\left\langle\left(\frac{\partial u_{1}}{\partial x_{1}}\right)^{2}\right\rangle^{n / 2}} \sim R_{\lambda}^{\alpha_{n}}
$$

where

$$
\alpha_{n}=\frac{3}{4} \mu n(n-1)
$$

(Frenkiel and Klebanoff 1975). The $\beta$-model is similiar, but predicts that

$$
\alpha_{n}=\frac{3 \mu}{4-\mu}\left(\frac{n}{2}-1\right)
$$

(Nelkin and Bell, 1978). The success of these models in predicting the experimentally observed dependence of derivative skewness and flatness on Reynolds number is discussed in detail by Antonia, Satyaprakash, and Hussain (1982), who conclude that the lognormal model is superior in this respect. Neither of the corrections to the $k^{-5 / 3}$ law proposed has been observed, although the lognormal correction is much smaller and probably not much above statistical noise.

The value of $\mu$ can be found either by direct measurements or by using these models to calculate back from $\alpha_{n}$. Nelkin (1981) and Antonia, Satyaprakash, and Hussain (1982) summarize the current evidence and conclude that most previous estimates were too high. They suggest that $\mu=0.2$.

Structural models assume that the small scales are composed of tubes or sheets of vorticity. Corrsin (1962) assumed that sheets dominated and concluded that $\alpha_{4}=1.5$. Saffman (1968) also assumed that the vorticity would be found in sheets whose thickness would be the order of the Taylor microscale (9), but that within the sheets, dissipation would be localized in regions characterized by the Kolmogorov length scale (10a). He found that $\alpha_{4}=1$ and $\alpha_{3}=0$, that is, the skewness is constant. A variation, proposed by Tennekes (1968), assumes that the dominant structures are tubes whose thickness is the Kolmogorov length scale, but which are subject only to the large-scale strain. His results agree with those of Saffman. Experimentally, $\alpha_{3}$ is observed to be very small, possibly zero, but $\alpha_{4}$ is much less than the predicted value of one. 
Dissipation of the variance of a passive scalar is observed to be intermittent, much like kinetic-energy dissipation. For example, the scalar-derivative flatness scales with Reynolds number in a manner similiar to the velocityderivative flatness, but with a larger exponent (Antonia and Chambers 1980). Sudden jumps in experimental temperature signals, known as "ramps", are also observed (Antonia et al. 1979). The only theoretical attempt to describe scalar intermittency was by Van Atta (1974), who used lognormal assumptions. Correlations between the velocity and scalar derivatives would also be interesting, but because it is difficult to measure two turbulent fields simultaneously, there have been almost no experimental measurements of these correlations.

In this paper, a variety of velocity- and scalar-derivative correlations are calculated over a range of Reynolds numbers. In an isotropic uncorrelated field with Gaussian statistics each of the correlations to be discussed has an easily determined value. For the velocity-derivative correlations $F_{\partial u^{n}}$ (3), the skewness $(n=3)$ is zero, the flatness $(n=4)$ is 3 , and the sixth order correlation is 15 . For mixed correlations in which both the velocity derivative and the scalar derivative have a power of 2, such as the mixed-derivative correlation (40), the uncorrelated value is 1. At high Reynolds numbers the statistics are usually highly non-Gaussian, both in experiments and in our simulations. We will concentrate on third- and fourth-order correlations, with some fifth- and sixth-order correlations presented to allow comparisons with the phenomenological models. Since the models discussed predict scaling laws and exponents, we will estimate these exponents and make comparisons.

The governing equations are the incompressible Navier-Stokes equation for the velocity and the transport equation for a passive scalar. The NavierStokes equation is

$$
\begin{gathered}
\partial u / \partial t+u \cdot \nabla u=-\nabla p / \rho+\nu \nabla^{2} u \\
\nabla \cdot u=0 \quad \text { (incompressibility) }
\end{gathered}
$$

The nonlinear term, $u \cdot \nabla u$, can be written in several different forms which are computationally convenient: the conservative form,

$$
\nabla \cdot(u u)
$$

and the rotational form,

$$
\omega \times u+\nabla \frac{1}{2} u^{2}
$$


The scalar equation can also be written two ways in incompressible flow: the convective form

$$
\partial \vartheta / \partial t+u \cdot \nabla \vartheta=D \nabla^{2} \vartheta
$$

and the conservative form

$$
\partial \vartheta / \partial t+\nabla \cdot u \vartheta=D \nabla^{2} \vartheta
$$

In the absence of viscosity $\nu$ and diffusivity $D$ the equations conserve two positive-definite quadratic invariants: the kinetic energy of turbulent fluctuations,

$$
E=\frac{1}{2}<u_{i} u_{i}>
$$

and the scalar variance,

$$
E_{\theta}=<\theta^{2}>
$$

where the domain of integration is over all space. The fundamental dimensionless parameters that we use are the Taylor-microscale Reynolds number, $R_{\lambda}=\mathrm{U} \lambda / \nu$, and the Prandtl number $\nu / D$, where $U$ is the characteristic velocity of the turbulence,

$$
\frac{3}{2} U^{2}=E
$$

and $\lambda$ is the Taylor microscale,

$$
\left.\lambda=\left\langle u_{1}^{2}\right\rangle^{1 / 2} /<\left(\partial u_{1} / \partial x_{1}\right)^{2}\right\rangle^{1 / 2}
$$

Also of interest are the kinetic-energy dissipation rate,

$$
\epsilon=-\frac{d}{d t}<\frac{3}{2} U^{2}>
$$

and the scalar-variance dissipation rate,

$$
\chi=-\frac{d}{d t}<\theta^{2}>
$$

The Kolmogorov length scale, wavenumber cutoff, and velocity scale are defined as

$$
\eta=\left(\frac{\nu^{3}}{\epsilon}\right)^{\frac{1}{4}}, \quad K_{k}=\frac{1}{\eta}, \quad \text { and } \quad(\epsilon \nu)^{1 / 2}
$$

and the Oboukov-Corrsin microscale and wavenumber (Corrsin 1951) are defined as

$$
\eta_{O C}=\left(\frac{D^{3}}{\epsilon}\right)^{\frac{1}{4}} \quad \text { and } \quad K_{O C}=\frac{1}{\eta_{O C}}
$$




\section{$\S B$. Numerical Method}

Two numerical codes were used for the simulations presented. Each is a three-dimensional spectral code with periodic boundary conditions. By spectral we mean that the fundamental variables that are stored and advanced in time are the Fourier-transformed velocity and scalar fields, $u(k)$ and $\theta(k)$. For simple geometries these methods allow more resolution than finite-difference methods for the same number of grid points (Orszag 1971). To improve the speed of spectral methods fast Fourier transforms are used to return to physical space, where the nonlinear terms are calculated by forming products. This method is sometimes referred to as pseudospectral because it introduces aliasing errors in the high wavenumbers. One way to reduce these errors, or dealias, is by truncating interactions outside a boundary in Fourier space. This does not significantly affect the resolution because only the highwavenumber tail of the spectrum, with only a small fraction of the total energy, is truncated. To completely dealias one should truncate all wavenumbers larger than $N / 3$, where $N$ is the number of grid points in a single direction. To completely dealias a three-dimensional code without truncation requires calculating the nonlinear terms on eight shifted grids. Fortunately, Patterson and Orszag (1971) have shown that in two or more dimensions all the aliasing errors can be eliminated by using only two shifted grids and a spherical truncation with the proper radius. This method was used in their original code, known as Superbox. Another approach uses shifted grids on alternate evaluations (Rogallo, 1981). The extent to which aliasing errors affect a calculation also depends on the algorithm used for the convective terms. Superbox uses the aliased rotation algorithm (6c) because it conserves kinetic energy, which helps inhibit instabilities and reduces the need for using shifted grids. Comparisons between versions of Superbox with and without shifted grids show no significant differences in their spectra (Patterson 1980).

The scalar equations do not allow aliasing to be neglected as easily as the Navier-Stokes equations do. For zero diffusivity, the scalar variance is conserved by the exact equations. But the aliased versions of both the convective and conservative equations $(7 a, b)$ do not conserve scalar variance. Instead, their respective aliasing errors are equal in magnitude and opposite in sign. We investigated taking advantage of this by averaging the two forms of the scalar equation to dealias, but it did not yield any significant improvements because our fields were already well resolved. Arakawa (1962) used similiar considerations in writing his stable finite-difference scheme for the two-dimensional Navier-Stokes equations. 
The code used for the $32^{3}$ runs is based on the Superbox code of Siggia and Patterson (1978) and Siggia (1981a). The rotational form of the NavierStokes equation (6c) was used for the velocity and the time advancement was leapfrog with stabilization done every 40 time-steps by a second-order RungeKutta step. Shifted grids were not used for dealiasing, but the convective and conservative forms of the scalar equation $(7 \mathrm{a}, \mathrm{b})$ were used on alternate timesteps to partially dealias the scalar. Wavenumbers were truncated outside a sphere of radius $N / 2$. This truncation has the advantage of being isotropic, but does allow significant one-dimensional aliasing errors. Details of this code may be found in Kerr (1981). Simulation of the velocity and one scalar required approximately 0.75 second of computer time (cpu) per evaluation on a Cray-1S computer and the longest simulation used 40 minutes of computer time.

The code for the $64^{3}$ and $128^{3}$ calculations also used the rotational form of the Navier-Stokes equations, with a spherical truncation of $N / 2$ and without shifted grids, but only the conservative form of the scalar equation was used. The time advancement was third-order Runge-Kutta (Wray 1981) and up to three scalars could be calculated simultaneously. Two-to-one word packing was used to improve data management (оr I/O). For a $64^{3}$ mesh with three scalars, 6.5 seconds of Cray cpu time and 2.0 seconds of I/O wait (time waiting for retrieval of data from disks) were required each evaluation, with three evaluations per time-step. The code with no scalars required 3.25 seconds of cpu time and 1 second of I/O wait per evaluation. Our longest simulation for a $64^{3}$ mesh with three scalars (F 19-21) required 12 hours of computer time to simulate seven eddy-turnover times (11).

We tried to run a $128^{3}$ mesh using Fortran I/O on the Cray-1S with disks, but due to the large number of files on disk, I/O wait became intolerably large. What made possible a $128^{3}$ simulation was the installation of a solid-state storage device (SSD), which effectively expanded the memory of the Cray-1S to 17 million words. For a $128^{3}$ mesh with three scalars approximately 55 seconds of cpu time and a negligible amount of time waiting for I/O between the SSD and main memory were required per evaluation. Three eddy-turnover times required 70 hours of Cray time.

\section{$\S \mathrm{C}$. Forcing, Simulation Parameters, and Aliasing Limits}

The codes outlined have been used for forced and decaying simulations. This paper will concentrate on the higher-order correlations obtained when the large scales are forced. The decaying calculations are discussed in detail by Herring and Kerr (1982) and Kerr (1981), though some of those results 
are included here in figure 3 and table 3.

Why do we force the large scales? Ideally we would like to simulate an infinite domain with unlimited resolution of the smallest scales, but in practice we are limited to a finite box with a finite mesh. There is a minimum wavenumber associated with the size of the box and a maximum wavenumber associated with the mesh spacing. If the effects of these limits are to minimized, the energy must be restricted to a very small range of wavenumbers and the Reynolds number must be small. The calculations discussed in Herring and Kerr (1982) simulate decaying flow behind a grid and are restricted in this way.

By forcing the large scales, some of the restrictions imposed by the lowwavenumber limit can be removed. It is believed that for sufficiently large Reynolds number that there is a cascade of energy from large to small scales which maintains a statistically-steady state. By forcing we hope to mimic a cascade from wavenumbers smaller than the minimum wavenumber to those in our computational box. Forcing would also maintain a steady spectrum at moderate Reynolds numbers and allow us to collect statistics of the higherorder correlations. Since the derivative correlations are representative of the small scales or high wavenumbers, it is hoped that they will not be affected by the details of the large scales, in our case the details of the artificial forcing used. Our approach is opposite to that of many turbulence modelers, who are primarily interested in the large scales and model the small scales.

Both the velocity and scalar were forced by time advancing the lowest wavenumber band $\left(1 \leq k / k_{o}<2\right.$, where $k_{o}$ is the lowest wavenumber of the code) independently of the high wavenumbers. The modes calculated in this manner were included in the full equations and the higher modes were advanced as usual. Details of the numerics may be found in Kerr (1981). Analysis of single modes in the forced wavenumber band indicates that the modes behave chaotically, although a complicated periodic motion cannot be ruled out. Our forcing is different than that used by Siggia (1981a), whose forcing modes were outside his computational box.

We present results from 27 forced cases for the Taylor-microscale Reynolds number, $R_{\lambda}$, between 9 and 82.9 and the Prandtl number between 0.1 and 2.0 (see table 1), but will discuss only a few in detail. All but one of the simulations includes a passive scalar. The exception (labeled F12 in table 1) uses the original dealiased Superbox code and our forcing to discern the effect of aliasing.

Each simulation was run until a statistically-steady state was reached, 
usually at about two eddy-turnover times. An eddy-turnover time is defined as

$$
t_{e}=\frac{L}{U}
$$

where

$$
L=\frac{3 \pi}{4 E} \int k^{-1} E(k) d k
$$

is the integral length scale. After reaching a steady state, statistics were collected for at least one and a half more eddy-turnover times and the means and variances of a variety of velocity and scalar correlations were obtained by averaging. These statistics and the errors are discussed in detail in appendix A.

Most earlier $32^{3}$ spectral simulations (Orszag and Patterson 1972) have been limited to $R_{\lambda}$ less than 30 based on considerations of the location of the peak of the three-dimensional velocity-dissipation spectrum (which is near the "bump" discussed in the following section for figure 1). Further restrictions come from considerations of trends in the derivative flatnesses. Generally the derivative skewnesses $(14,15)$ and flatnesses $(32,39)$ increase, or remain constant, with increasing Reynolds number. Failure to do so indicates that small-scale truncation and aliasing errors are significant. This behavior was observed for a $32^{3}$ mesh at Reynolds numbers larger than 24 . In order to demonstrate the magnitude of aliasing errors at this Reynolds number, table $2 \mathrm{~A}$ gives derivative flatness factors for $32^{3}$ and $64^{3}$ calculations starting with identical initial conditions at $R_{\lambda} \approx 29$. On the $64^{3}$ grid there are virtually no aliasing or truncation errors at this Reynolds number for any of the Prandtl numbers used. Any differences between the $32^{3}$ and $64^{3}$ results are due to aliasing and high-wavenumber truncation errors in the $32^{3}$ simulation. In run F11 only spherical truncation is used to dealias; while F12 uses the original Superbox code to dealias by shifting grids on each evaluation. For F11(aliased) both the velocity-derivative flatness and scalar-derivative flatness for $\operatorname{Pr}=0.5$ are significantly lower than the $64^{3}$ results (F14), while in F12(dealiased) there is virtually no difference with F14. Therefore, at this Reynolds number an aliased $32^{3}$ calculation does not resolve the small scales, whereas a dealiased calculation does.

To estimate the maximum allowable Reynolds numbers on the $64^{3}$ and $128^{3}$ grids, the Kolmogorov wavenumber cutoffs of the simulations can be compared to the maximum wavenumbers allowed by the grids. For the inertial range $\left(E(k) \sim K_{o} \epsilon^{2 / 3} k^{-5 / 3}\right) R_{\lambda} \sim K_{k}^{\frac{2}{3}}$. But the Reynolds numbers in 
the $32^{3}$ and $64^{3}$ simulations are too low for an inertial range to appear and $R_{\lambda} \sim K_{k}{ }^{1.25}$ fits our results better. If the maximum allowable Kolmogorov wavenumber on a $64^{3}$ grid is assumed to be twice that permitted on a $32^{3}$ grid, then to ensure that the small scales in the $64^{3}$ simulation are fully resolved the maximum $R_{\lambda}$ is $55.9\left(\simeq 24 \times 2^{1.25}\right)$. As shown in figure 1 , our $128^{3}$ simulation has a noticeable $-5 / 3$ inertial range, so the $K_{k}{ }^{\frac{2}{3}}$ law should be used and $R_{\lambda}=82.9\left(\simeq 52 \times 2^{0.67}\right)$.

In addition to its effect on the calculation of the velocity and scalar fields, aliasing can introduce errors in the calculation of the derivative flatness factors. To estimate this error the derivative flatnesses for a $64^{3}$ simulation were calculated on a $32^{3}$ grid for one realization. Table $2 \mathrm{~B}$ shows that the velocity-derivative flatness calculated on a $32^{3}$ grid yields a slightly smaller result. This reinforces our conclusion that $R_{\lambda} \sim 29$ is slightly too high for a $32^{3}$ grid. The scalar-derivative flatness for $\operatorname{Pr}=0.5$ shows aliasing errors similiar to those in the velocity field, so no further restrictions on the Reynolds number are necessary when $P r \leq 0.5$. On the other hand, a significant decrease is seen in the scalar-derivative flatness for $\operatorname{Pr}=1.0$ when it is calculated on a $32^{3}$ grid.

Naively one would expect that for Prandtl number of 1 , where the viscosity and scalar diffusivity are the same, that there would be similiar truncation effects for the velocity and the scalar. Instead, the scalar errors are much larger. What does theory tell us? For Prandtl number greater than 1, Batchelor (1959) predicts a new spectral regime in which scalar variance diminishes as $k^{-1}$ for wavenumbers greater than the Kolmogorov wavenumber. The cutoff for the new spectral regime is $k_{B}=\left(\epsilon / \nu D^{2}\right)^{1 / 4}$. This spectral regime has been observed (see Monin and Yaglom 1975, p.513). Since a higher Prandtl number implies a higher spectral cutoff, for large Prandtl numbers truncation effects will appear even at very low Reynolds numbers. Consequently for $\mathrm{Pr}=2, R_{\lambda}>12$ was not run on a $32^{3}$ mesh. But an anomalous $k^{-1}$ subrange is also observed experimentally for Prandtl number as low as 0.7, the atmospheric value (the "bump", Hill 1978). Therefore, even at $\mathrm{Pr}=1$ truncation, as indicated by the scalar-derivative flatness, becomes important for $R_{\lambda}>18$ on a $32^{3}$ grid.

Because of these difficulties our simulations are restricted to low Prandtl numbers. Some of the simulations for $\mathrm{Pr}=1$ are included in the plots of the correlations, but only for lower Reynolds numbers. Prandtl numbers of current experimental interest that we can simulate are 0.7 , the atmospheric value, and moderately low Prandtl numbers, such as found in liquid metals. 
Extremely low values cannot be simulated satisfactorily because the relevant low-wavenumber cutoff is the Oboukov-Corrsin cutoff, $K_{O C}(10 \mathrm{~b})$, and this would be below the minimum wavenumber of the simulation. The lowest value we use is $\operatorname{Pr}=0.1$. Analysis of spectra in the next section support these conclusions.

\section{$\S D$. Spectra and Skewnesses}

Since most simulators determine whether there is significant truncation error by inspecting high-wavenumber spectra, we have included some spectra to demonstrate the validity of our method. Figure 1 shows three-dimensional energy spectra, normalized by the Kolmogorov microscales and multiplied by $k^{5 / 3}$, for several Reynolds numbers. The turnup at large wavenumbers is a numerical effect of the large-wavenumber cutoff. Where the turnup is small, the small scales are well resolved. The turnup for $R_{\lambda}=55.9$ in a $64^{3}$ simulation and $R_{\lambda}=82.9$ in the $128^{3}$ simulation is the most we could accept. The three-dimensional kinetic-energy and scalar-variance spectra for our largest Reynolds number, 82.9, are plotted in figure 2. The velocity spectrum is normalized as in figure 1 and the scalar spectra are normalized by the Kolmogorov microscales and the scalar-variance dissipation in a similiar manner. When compared with the scalar variance at low wavenumbers, the turnup at high wavenumbers for $P r=1$ is unacceptably large. This reinforces our conclusion that moderate-to-high Prandtl numbers can be simulated only with low-Reynolds-number simulations.

With the exception of deviations in the lowest Reynolds-number spectrum $\left(R_{\lambda}=18.5\right)$, all of the spectra in figure 1 collapse to a single curve in the dissipation range. In addition, a short $-5 / 3$ regime appears in the $128^{3}$ spectrum $\left(R_{\lambda}=82.9\right)$ at low wavenumbers. For comparison, the dashed line in figure 1 fits a spectrum of the form predicted by Pao (1965),

$$
E(k)=K_{o} \epsilon^{2 / 3} k^{-5 / 3} \exp \left(-1.5 K_{o} \nu \epsilon^{-1 / 3} k^{4 / 3}\right)
$$

to the computed spectra with $K_{o}$, the Kolmogorov constant, equal to 2.45. Although experimental values for the Kolmogorov constant as high as 2.1 have been reported (Gibson et al. 1970), the fitted value is much higher than the usual experimental value of 1.4 to 1.7 (Monin and Yaglom 1975, p.483-485). To understand the apparent differences between the calculated and experimental Kolmogorov constant the velocity-derivative skewness must be considered. From this we will conclude that fitting the calculated spectra with Pao's form could lead to anomalously high values for the Kolmogorov constant. 
For both the velocity and scalar three classes of the derivative skewnesses can be defined. These are the real-space derivative skewnesses, spectraltransfer skewnesses, and dissipation skewnesses. The real-space derivative skewnesses are measurable with hot-wire probes. The spectral and dissipation skewnesses are not strictly third-order moments, but in isotropic flow they are related to the real-space derivative skewnesses. The measurable real-space derivative skewnesses are the velocity-derivative skewness,

$$
S_{u}=\frac{\left\langle\left(\frac{\partial u_{1}}{\partial x_{1}}\right)^{3}\right\rangle}{\left.<\left(\frac{\partial u_{1}}{\partial x_{1}}\right)^{2}\right\rangle^{\frac{3}{2}}}=F_{\partial u^{3}}
$$

the mixed-derivative skewness

$$
S_{u \theta}=\frac{\left\langle\frac{\partial u_{1}}{\partial x_{1}}\left(\frac{\partial \theta}{\partial x_{1}}\right)^{2}\right\rangle}{\left\langle\left(\frac{\partial u_{1}}{\partial x_{1}}\right)^{2}\right\rangle^{\frac{1}{2}}\left\langle\left(\frac{\partial \theta}{\partial x_{1}}\right)^{2}\right\rangle}
$$

and the scalar-derivative skewness

$$
S_{\theta}=\frac{\left\langle\left(\frac{\partial \theta}{\partial x_{1}}\right)^{3}\right\rangle}{\left\langle\left(\frac{\partial \theta}{\partial x_{1}}\right)^{2}\right\rangle^{\frac{3}{2}}}
$$

In isotropic turbulence the terms in the velocity-derivative skewness and the mixed-derivative skewness can be written more generally. That is,

$$
\begin{gathered}
\frac{35}{2}<\left(\frac{\partial u_{1}}{\partial x_{1}}\right)^{3}>= \\
\frac{4}{3}<\operatorname{tr} \quad e^{3}>= \\
-<\omega_{i} e_{i j} \omega_{j}>
\end{gathered}
$$

(Betchov 1956) and

$$
\frac{15}{2}<\frac{\partial u_{1}}{\partial x_{1}}\left(\frac{\partial \theta}{\partial x_{1}}\right)^{2}>=<\theta_{i} e_{i j} \theta_{j}>
$$

Furthermore,

$$
<\left(\partial u_{1} / \partial x_{1}\right)^{2}>=\frac{1}{15} \epsilon / \nu
$$


and

$$
<\left(\frac{\partial \theta}{\partial x_{1}}\right)^{2}>=\frac{\chi}{6 D}
$$

These rotationally-invariant forms are used in all our calculations of the realspace skewnesses.

The spectral-transfer skewnesses and the dissipation skewnesses are related to the real-space skewnesses through integral equations for the dissipation of energy and scalar variance,

$$
\frac{1}{2 \nu} \frac{d}{d t} \epsilon=\int k^{2} T_{u}(k) d k-2 \nu \int k^{4} E_{u}(k) d k
$$

and

$$
\frac{1}{2 D} \frac{d}{d t} \chi=\int k^{2} T_{\theta}(k) d k-D \int k^{4} E_{\theta}(k) d k
$$

where $T_{u}(k)$ and $T_{\theta}(k)$ are the nonlinear energy and scalar-variance transfers, respectively. For our forced equations another term should be added, but this term is negligible except at low Reynolds numbers. The secondorder moments of the transfers are related to the real-space skewnesses by $\int k^{2} T_{u}(k) d k=\left\langle\omega_{i} e_{i j} \omega_{j}>\right.$ and $\int k^{2} T_{\theta}(k) d k=-<\theta_{i} e_{i j} \theta_{j}>$, identically. The integrals of the transfers are zero $\left(\int T(k) d k=0\right)$, but because there is a net cascade of kinetic energy and scalar variance to higher wavenumbers, their second-order moments will in general be positive and nonzero. Therefore, we can define two spectral-transfer skewnesses, the velocity spectral skewness

$$
S_{u(k)}=\frac{2}{35} \frac{\int k^{2} T_{u}(k) d k}{\left(\frac{\epsilon}{15 \nu}\right)^{\frac{3}{2}}}=\frac{2}{35} \frac{P_{\Omega}}{\left(\frac{\epsilon}{15 \nu}\right)^{\frac{3}{2}}}
$$

and the mixed spectral skewness

$$
S_{\theta(k)}=\frac{2}{15} \frac{\int k^{2} T_{\theta}(k) d k}{\left(\frac{\epsilon}{15 \nu}\right)^{\frac{1}{2}}\left(\frac{\chi}{6 D}\right)}
$$

where $P_{\Omega}$ is the rate of enstrophy production and $\Omega$, the enstrophy, equals $\frac{1}{2}<\omega_{i} \omega_{i}>$. Because the spectral-transfer skewnesses are nonzero, in isotropic flow the velocity- and mixed-derivative skewnesses will also be nonzero and obey

$$
S_{u}=-S_{u(k)} \quad S_{u \theta}=-S_{\theta(k)}
$$


These arguments do not apply to the real-space scalar-derivative skewness (16), which in an isotropic calculation such as ours is zero. However, it has recently been found that in shear flows with a temperature gradient that the scalar-derivative skewness is nonzero (Sreenivasan and Tavoularis 1980).

For our statistically-steady forced simulations $\frac{d}{d t} \epsilon \rightarrow 0$ and $\frac{d}{d t} \chi \rightarrow 0$, so we expect that as the Reynolds numbers increases that $\int k^{2} T_{u} \rightarrow \nu \int k^{4} E_{u}$ and $\int k^{2} T_{\theta} \rightarrow D \int k^{4} E_{\theta}$. Therefore, a third measure of the skewnesses, the dissipation skewnesses, can be defined by (Wyngaard 1971)

$$
S_{\epsilon}=\frac{2}{35} \frac{\epsilon \Omega}{\left(\frac{\epsilon}{15 \nu}\right)^{\frac{3}{2}}}
$$

and

$$
S_{\chi}=\frac{2}{15} D \frac{\int k^{4} E_{\theta}(k) d k}{\left(\frac{\epsilon}{15 \nu}\right)^{\frac{1}{2}}\left(\frac{\chi}{6 D}\right)}
$$

where

$$
\epsilon_{\Omega}=-\frac{d \Omega}{d t}=2 \nu \int k^{4} E(k) d k
$$

is the enstrophy dissipation rate.

As discussed in the introduction, all the higher-order correlations are expected to diverge from their uncorrelated values as the Reynolds number increases. Experimentally, the velocity-derivative skewness increases from zero, the uncorrelated value, to a value greater than 0.3 at very low experimental Reynolds numbers and appears to increase indefinitely in very high Reynoldsnumbers atmospheric measurements (Tavoularis et al. 1978). But at intermediate Reynolds numbers the experiments are less conclusive. Tavoularis, Bennett, and Corrsin (1978) cite data which suggests that the skewness decreases with Reynolds number between $R_{\lambda}=10$ and 500. Klebanoff (1982) finds the velocity-derivative skewness to be constant and equal to 0.43 between $R_{\lambda}=40$ and 200 in a boundary layer. This experimental result is plotted in figure 3 .

Simulations discussed in Herring and Kerr (1982) show that the velocityderivative skewness increases from zero to 0.4 by $R_{\lambda} \sim 20$, in agreement with low-Reynolds-number experiments. They find that the mixed-derivative skewness increases in a similiar manner. At intermediate Reynolds numbers, they suggest that both the velocity- and mixed-derivative skewnesses reach maximum values, then remain constant with increasing Reynolds number, agreeing with Klebanoff"s experiment. With our $64^{3}$ and $128^{3}$ simulations we 
have determined the skewnesses at higher Reynolds numbers and confirmed those results (figure 3). Figures 3 and 4 plot the real-space and dissipation skewnesses, respectively, as functions of $R_{\lambda}$ and in all cases the magnitudes of the skewnesses converge to approximately 0.5 . The value for the velocityderivative skewness for simulations F11 to F27 is $-0.505 \pm 0.005$.

The statistical models of the small scales and the atmospheric measurements mentioned predict that the velocity-derivative skewness will increase very slowly with Reynolds number $(4,5)$. Although the accuracy of our curve is not good enough to rule out the existence of such a trend, it appears significant that both our simulation and some experiments show no Reynoldsnumber dependence over a wide range. The reason the velocity-derivative skewness does not increase with Reynolds number is probably related to the alignment of small-scale structures. Recall that the model of Tennekes (1968) suggested that if the structures are tubes, then the skewness would not increase with Reynolds number. In section G (Graphics) it will be seen that our structures are tubes. In addition, fourth-order statistics and our graphics find strong alignment between the vorticity and the rate of strain.

Because of the connection between the skewnesses and the fourth-order moment of the spectra, a simple relation between the velocity-derivative skewness and the Kolmogorov constant exists. If one assumes Pao's spectral form (13) and uses the equation for the velocity-dissipation skewness(24), then

$$
S_{u}=-\frac{4}{35} 15^{\frac{3}{2}} \frac{9}{16} \sqrt{ } \pi\left(\frac{3}{2} K_{o}\right)^{\frac{-3}{2}}=-2.40 K_{o}{ }^{\frac{-3}{2}}
$$

For $S_{u}=-0.5, K_{o}=2.85$. For a typical experimental Kolmogorov constant, $K_{o}=1.7,(27)$ gives $S_{u}=-1.08$, which disagrees with experimental measurements of the skewness at moderate Reynolds numbers. If the experimental values for the Kolmogorov constant and the skewness are correct, then Pao's spectral form is clearly a poor approximation to the experimental spectra. There are also significant differences between the calculated spectra of figure 1 and Pao's form near $(\eta k) \sim 0.2$. If the calculated Kolmogorov constant is to be consistent with the experiments (that is $K_{0} \sim 1.7$ ), then at wavenumbers below those simulated, the correct spectrum must be below Pao's form for $K_{0}=2.45$. The skewness would not change in this case because the major contribution to it comes from the wavenumber regime already simulated. Experimental spectra by Champagne et al. (1977) show a high wavenumber bump which is similiar to the computed spectral shape. Additional experiments which determine both the Kolmogorov constant and 
the skewness and make detailed comparisons with Pao's spectral form would help answer these questions.

There are no experiments or phenomenological models with which to compare our results on the dependence of the mixed-derivative skewness with Reynolds number. But there are arguments that the mixed-derivative skewness should increase with Prandtl number, possibly with an upper bound at high Prandtl number for given Reynolds number (Hill 1978). Hill's arguments assume that there are two spectral regimes for the scalar variance at low Prandtl numbers, a $k^{\frac{-5}{3}}$ inertial regime and a $k^{\frac{-17}{3}}$ inertial-diffusive regime. There are strong theoretical arguments in favor of both spectral regimes (Corrsin 1951 and Batchelor et al. 1959). Experimentally, a $k^{\frac{-5}{3}}$ inertial range for the scalar variance has been well demonstrated (Monin and Yaglom 1975 , p.511), but the evidence for a $k^{\frac{-17}{3}}$ inertial-diffusive regime is tenuous. This is due in part to the exotic nature of low-Prandtl-number materials, such as liquid mercury and sodium. Experimental results on the dependence of the mixed-derivative skewness on Prandtl number are also difficult to obtain. With the exception of a few measurements in air $(\mathrm{Pr}=0.7$ in figure 4) the only measurements of the mixed-derivative skewness were taken in water and liquid mercury by Clay (1973). For air, measurements in the atmosphere (Antonia and Van Atta, 1978) give much larger values than those in wind tunnels. Our results, Hill's model \#4, the test field model (Larcheveque et al. 1980), and several experimental values (taken from Larcheveque et al.) are shown in figure 5. Our values are the maximum $S_{u \theta}$ for a given Prandtl number in our simulations (see figure 3). The experiments of Clay (1973) suggest that there is virtually no dependence on Prandtl number, which is compatible with our results. Figure 3 suggests that for higher Reynolds numbers $S_{u \theta}$ will asymptote to -0.5 for $P r=0.1$, but remain constant for $P r=$ 0.5 and 1.0. In this case, simulations at higher Reynolds numbers should show that the mixed-derivative skewness is constant over a wide range of Prandtl numbers. A long crossover regime between the $k^{\frac{-5}{3}}$ and $k^{\frac{-17}{3}}$ spectral ranges could explain this result. Gibson (1968) has suggested that this new regime should go as $k^{-3}$, but the spectrum for $\operatorname{Pr}=0.1$ in figure 2 does not support this.

\section{$\S \mathrm{E}$. Fourth- and Higher-Order Velocity Correlations}

Siggia (1981b) has shown that in isotropic flow all fourth-order velocityderivative correlations can be expressed in terms of four rotational invariants of the velocity-deformation tensor. These are 


$$
\begin{gathered}
I_{1}=<e^{4}> \\
I_{2}=<\omega^{2} e^{2}> \\
I_{3}=<\omega_{i} e_{i j} e_{j k} \omega_{k}>
\end{gathered}
$$

and

$$
I_{4}=\left\langle\omega^{4}\right\rangle
$$

where $e_{i j}=\frac{1}{2}\left(\frac{\partial u_{i}}{\partial x_{j}}+\frac{\partial u_{j}}{\partial x_{i}}\right)$ is the rate of strain, $e^{2}=\sum e_{i j}{ }^{2}, e^{4}=\left(e^{2}\right)^{2}$ , $\omega_{i}=\nabla \times u_{i}$ is the vorticity, and $\omega^{2}=\sum \omega_{i}{ }^{2}$. We will normalize these correlations as follows:

$$
\begin{gathered}
F_{1}=\frac{15}{7} \frac{I_{1}}{\left.<e^{2}\right\rangle^{2}} \\
F_{2}=3 \frac{I_{2}}{<\omega^{2}><e^{2}>} \\
F_{3}=3 \frac{I_{3}}{\left.<\omega^{2}><e^{2}\right\rangle}
\end{gathered}
$$

and

$$
F_{4}=\frac{9}{5} \frac{I_{4}}{\left.<\omega^{2}\right\rangle^{2}}
$$

Their uncorrelated values are $3,3,1$, and 3 , respectively, and they are plotted in figure 6.

The only fourth-order velocity-derivative correlation that has been experimentally determined is the velocity-derivative flatness, $F_{\partial u^{4}}(3)$, which equals $F_{1}$ if isotropy is assumed. Kuo and Corrsin (1971) find that $F_{\partial u^{4}}$ increases with Reynolds number as $R_{\lambda}{ }^{\alpha_{4}}$ with $\alpha_{4}=0.25$ for $12<R_{\lambda}<100$. Our flatness ( $F_{1}$ or $F_{\partial u^{4}}$, figures 6,7 and 8 ) also increases with Reynolds number and is in excellent agreement with the experiment of Frenkiel and Klebanoff (1975) (upper dotted line, figure 7), but $\alpha_{4}=0.18 \pm 0.03$.

If only one scaling exponent is necessary to describe intermittency (as suggested by the phenomenological models), all the fourth-order velocityderivative correlations should exhibit similiar behavior. Correlations $F_{1}$ to $F_{4}$ have been calculated for the forced runs and plotted as functions of Reynolds number in figure 6 . If power laws are fitted to $F_{1}, F_{2}$, and $F_{4}$ 
for $R_{\lambda}$ greater than 28 , the exponents are $0.18 \pm 0.03,0.29 \pm 0.03$, and $0.37 \pm 0.03$, respectively. This suggests that not one, but at least two scaling exponents are necessary, one for the rate of strain and the other for the vorticity. Furthermore, $F_{3}$ decreases noticeably with Reynolds number at very low Reynolds number and rises only a small amount at higher Reynolds numbers. The low value for $F_{3}$ suggests that the principal component of the rate of strain and the vorticity tend to be perpendicular to each other, which would always be the case in two dimensions, and implies that there is strong alignment in the small-scale turbulent structures. This is demonstrated by the graphics discussed in section $\mathrm{G}$. The similiarity between one of the isotropic forms of the skewness (17b) and $I_{3}$ suggests that the low value for $F_{3}$ is related to the invariance of the velocity-derivative skewness.

Although the computed fourth-order correlations suggest that several scaling exponents are necessary, it is possible that at much higher Reynolds numbers one scaling exponent is sufficient. If so, the self-similiar regime described by the models has not been reached. The slight rise in $F_{3}$ might indicate that the calculations are approaching this regime. A change in the velocityderivative flatness scaling exponent at higher Reynolds numbers would be seen as all the exponents approach the asymtotic value. This could be interpreted as a variation in the dissipation-dissipation correlation exponent $\mu$ with Reynolds number. Experimentally, the Reynolds-number dependence of the velocity-derivative skewness and flatness is different at very high Reynolds number, with $\alpha_{4} \sim 0.32$ for $R_{\lambda}=200$ to 20,000 (Van Atta and Antonia 1980). There are two experimental measurements that would help clarify this matter. If $u_{2}$ and $u_{1}$ can be measured simultaneously, which now seems possible with crossed-wire probes, then

$$
I_{5}=<\left(\frac{\partial u_{1}}{\partial x_{1}}\right)^{2}\left(\frac{\partial u_{2}}{\partial x_{1}}\right)^{2}>=\frac{I_{1}}{105}+\frac{I_{2}}{70}-\frac{I_{3}}{105}
$$

and

$$
I_{6}=<\left(\frac{\partial u_{2}}{\partial x_{1}}\right)^{4}>=\frac{3}{140} I_{1}+\frac{11}{140} I_{2}-\frac{3}{35} I_{3}+\frac{1}{80} I_{4}
$$

could be measured. If the trends calculated are correct, $I_{2}$ should dominate in $I_{5}$, and $I_{4}$ should dominate in $I_{6}$. This implies that the scaling exponent of the normalized correlation of $I_{5}$ should be greater than that of $F_{\partial u^{4}}$, and the scaling exponent of the normalized correlation of $I_{6}$ should be the largest.

The fifth- through eighth-order velocity-derivative correlations (3) and vor- 
ticity moments,

$$
F_{\omega^{n}}=\frac{\left\langle\omega_{1}^{n}\right\rangle}{\left\langle\omega_{1}^{2}\right\rangle^{n / 2}}
$$

have also been calculated. Since the statistics deteriorate as the order of the correlations increases (appendix A), only the velocity-derivative skewness, flatness, and fifth- and sixth-order correlations are plotted in figure 7, even though the fifth- and sixth-order statistics are questionable. The exponents for the velocity-derivative correlations in figure 7 are $0.18 \pm 0.03,0.27 \pm$ 0.03 , and $0.55 \pm 0.03$ respectively. The lognormal theory predicts that these exponents should be proportional to $n(n-1)$ (equation 4) and the $\beta$ model predicts (n-2) (equation 5). The lognormal theory provides a better fit, as in the experiments (Antonia et al. 1982). The associated value of the dissipation-dissipation correlation exponent $\mu$ has not been calculated because the simulations are not in the Reynolds number regime where the experimental value is usually found.

Assuming isotropy, $F_{\omega^{4}}=F_{4}$. The most important point that can be made about the sixth-order vorticity correlation $\left(F_{\omega^{6}}\right.$, figure 9$)$ is that its scaling exponent (1.1土0.1) is much larger than the corresponding sixth-order velocity-derivative scaling exponent, just as was true for the fourth-order correlations.

\section{$\S$ F. Scalar and Mixed Scalar-Velocity Fourth-Order Derivative Correlations}

There are three fourth-order derivative correlations which have been measured experimentally, the velocity-derivative flatness, $F_{\partial u^{4}}(3)$, the scalarderivative flatness

$$
F_{\partial \theta \boldsymbol{n}}=\frac{\left\langle\left(\frac{\partial \theta}{\partial x_{1}}\right)^{n}\right\rangle}{\left\langle\left(\frac{\partial \theta}{\partial x_{1}}\right)^{2}\right\rangle^{n / 2}}, \quad n=4
$$

and the mixed-derivative correlation

$$
F_{u \theta}=\frac{\left\langle\left(\frac{\partial u_{1}}{\partial x_{1}}\right)^{2}\left(\frac{\partial \theta}{\partial x_{1}}\right)^{2}\right\rangle}{\left\langle\left(\frac{\partial u_{1}}{\partial x_{1}}\right)^{2}\right\rangle\left\langle\left(\frac{\partial \theta}{\partial x_{1}}\right)^{2}\right\rangle}
$$

The uncorrelated value for the velocity- and scalar-derivative flatnesses, assuming Gaussian statistics, is 3, and the uncorrelated value for the mixedderivative correlation is 1 . The rotationally-invariant form of the scalar- 
derivative flatness in isotropic flow is

$$
F_{\partial \theta^{4}}=F_{\nabla \theta}=\frac{9}{5} \frac{\left\langle\nabla \theta^{4}\right\rangle}{\left\langle\nabla \theta^{2}\right\rangle^{2}}
$$

In the experiment of Antonia and Chambers (1980), the atmospheric temperature-derivative flatness increases much faster with Reynolds number than the velocity-derivative flatness. Specifically, if $F_{\partial \theta^{4}} \sim R_{\lambda}{ }^{\alpha_{\theta}}$, then $\alpha_{\theta}=0.5$ for $100<R_{\lambda}<10,000$. In our simulations (figure 8), the scalarderivative flatness also has a stronger dependence on Reynolds number than the velocity-derivative flatness. In addition, the scalar-derivative flatness increases with Prandtl number for given Reynolds number, while the scaling exponents decrease. For Prandtl numbers 0.1, 0.5, and 1.0 we found $\alpha_{\theta}=$ $0.48,0.44$, and 0.36 . The trend for the flatness factors at low Prandtl numbers to increase faster with Reynolds number than at larger Prandtl numbers suggests that the large exponents for $P r=0.1$ and 0.5 might be transient, and that at higher Reynolds numbers they will have a exponent similiar to that for $\operatorname{Pr}=1.0$. That is, $\alpha_{\theta}=0.36$, which is the same order as the exponent for the vorticity flatness $\left(F_{4}\right)$, might be an upper bound for the scaling exponent at large Reynolds numbers for all Prandtl numbers.

Although the scalar-derivative flatness increases with $R_{\lambda}$ at similiar rates in experiment and in our simulations, the Reynolds numbers are very different. Sreenivasan et al. (1980) have measured the scalar-derivative flatness in a wind tunnel with temperature fluctuations produced by a heated screen and find $F_{\partial \theta^{4}}=5.5$. The Prandtl number is 0.7 and we believe $R_{\lambda} \approx 24 .\left(R_{\lambda}\right.$ was computed from their $u^{2}$ data and is consistent with similiar wind-tunnel experiments by Warhaft and Lumley (1978).) For $R_{\lambda} \approx 24$, their result is consistent with our calculations and lies on our curve for $\mathrm{Pr}=0.5$ in figure 8.

The sixth-order scalar-derivative correlations, $F_{\partial \theta^{6}}$, are plotted in figure 9 . The scaling exponents for Prandtl numbers 0.1, 0.5, and 1.0 are 1.48, 1.30, and 0.92 respectively, showing the same trends with Prandtl number as the fourth-order correlations.

Before disscussing the one experimentally-measurable mixed correlation we will consider some rotationally-invariant correlations between the velocity and scalar derivatives where both are second order. These are

$$
F_{\nabla \theta \omega 1}=\frac{\left\langle(\nabla \theta)^{2} \omega^{2}\right\rangle}{\left\langle\nabla \theta^{2}\right\rangle\left\langle\omega^{2}\right\rangle}
$$




$$
\begin{gathered}
F_{\nabla \theta \omega 2}=3 \frac{\left\langle(\nabla \theta \cdot \omega)^{2}\right\rangle}{\left.\left\langle\nabla \theta^{2}\right\rangle<\omega^{2}\right\rangle} \\
F_{\nabla \theta e 1}=\frac{\left\langle(\nabla \theta)^{2} e^{2}\right\rangle}{\left.\left\langle\nabla \theta^{2}\right\rangle<e^{2}\right\rangle}
\end{gathered}
$$

and

$$
F_{\nabla \theta e 2}=\frac{\left.3<\partial \theta / \partial x_{i} e_{i j} e_{j k} \partial \theta / \partial x_{k}\right\rangle}{\left\langle\nabla \theta^{2}><e^{2}\right\rangle}
$$

They have been normalized so that their uncorrelated values are 1 . Correlation $F_{\nabla \theta \omega 1}$ is plotted in figure 10 and is nearly 1, showing that there is little correlation between the magnitudes of the vorticity and scalar derivative. Correlation $F_{\nabla \theta \omega 2}$ is plotted in figure 11 and shows a strong anticorrelation between the direction of vorticity and the direction of the scalar gradient, which would be consistent with the scalar is being wound around vortex tubes. Batchelor (1952) predicts this result for the stretching of a volume element of fluid.

Both correlations between the strain rate and the scalar derivative are greater than one and show a tendency to increase with Reynolds number for $R_{\lambda}<30$, then remain constant or increase slowly, much like the skewnesses do. The experimentally-measurable mixed correlation is related to $F_{\nabla \theta e 1}$ and $F_{\nabla \theta e 2}$ by

$$
F_{u \theta}=\frac{\left\langle\left(\frac{\partial u_{1}}{\partial x_{1}}\right)^{2}\left(\frac{\partial \theta}{\partial x_{1}}\right)^{2}\right\rangle}{\left\langle\left(\frac{\partial u_{1}}{\partial x_{1}}\right)^{2}\right\rangle\left\langle\left(\frac{\partial \theta}{\partial x_{1}}\right)^{2}\right\rangle}=\frac{3}{7} F_{\nabla \theta e 1}+\frac{4}{7} F_{\nabla \theta e 2}
$$

and is plotted in figure 12(a). $F_{u \theta}$ has been measured by Park (1976) and is greater than one. Correlations $F_{\nabla \theta e 1}$ and $F_{\nabla \theta e 2}$ are plotted in figures 12(b) and $12(c)$ respectively.

We have calculated a wide assortment of other correlation factors, none of which show a significant divergence from their uncorrelated values. This is especially true for those with the scalar variance, such as

$$
\frac{<\theta^{4}>}{\left.<\theta^{2}\right\rangle^{2}}
$$

For both our simulation and experiment (Sreenivasan et al. 1980) the scalarvariance flatness is 3 , the Gaussian value. This is expected because, except for mixing, the scalar level is not affected by the dynamics although its derivatives are. 
$\S$ G. Graphics In Siggia (1981a) three-dimensional perspective plots of the vorticity field indicated the presence of vortex tubes. We have produced similiar graphics for the vorticity, scalar gradient, and principal rates of strain for a single time from run F19. These are shown in figures 13 to 18 . These figures are two-dimensional, black-and-white projections of what can be seen using colored graphics or a three-dimensional graphics terminal. In figures 13(a-c) short lines are used to show the direction of vorticity, with the length of the lines proportional to the magnitude of the vorticity. The vorticity is plotted at a grid point only if it was above a threshold, which is picked such that only $0.25 \%$, or about 500 , of the grid points of a $64^{3}$ mesh are plotted. The $0.25 \%$ value was picked so as to allow an assortment of structures to appear, but not so many that the pictures would be cluttered. Figure 13(b) represents the same flow field as figure 13(a), but rotated $90^{\circ}$ about the vertical axis. A strong concentration of vorticity extends most of the way across our computational box in both projections. Rotation shows that this structure is a tube. The vorticity in the gap indicated by the circle in figure 13(a) is just below the threshold picked. In figure 13(c) the threshold is lowered such that $1 \%$ of the grid points are plotted and this gap is filled. The circled area in figure 13(c) indicates where two tubes might be merging into one.

In figures $14(a, b)$ the lines represent the direction of the scalar gradient. The projections are the same as in figures $13(\mathrm{a}, \mathrm{b})$ and $0.25 \%$ of the grid points are plotted. The scalar gradient field appears to have well-defined structures, and although it is not very clear in figures $14(a, b)$, rotation shows that these structures are sheets. By comparing figures 13(a) and 14(a) we can see that in the vicinity of the largest vortex tube that the scalar gradient is perpendicular to the vorticity. This is consistent with our calculation of $F_{\nabla \theta \omega 2}(43)$, which suggested that the scalar gradient and vorticity are orthogonal.

To better visualize the correlation between vorticity and scalar gradient, two-dimensional slices of the vorticity and scalar gradient are plotted in figures 15 and 16. The location of the slices is indicated by the arrow in figure 13(a). In these pictures, the scalar gradient (figure 16) appears wound around the vortex core (figure 15). Two-dimensional vortex simulations which include scalar markers (Aref and Siggia 1980) and flow-visualization experiments (Winant and Browand 1974; Brown and Roshko 1974) also show the scalar marker winding around what are believed to be vortex cores.

In figures $17(a, b)$ the rate of strain is plotted for the same flow field and projections as in figures $13(\mathrm{a}, \mathrm{b})$. Since the rate of strain is a tensor, it cannot 
be described with a single line segment. However, by plotting only one of the principal rates of strain at a time, graphics can be produced that show the structure of the strain field. In figures $17(a, b)$ we conditionally sample based on the trace of the square of the rate-of-strain tensor $\left(e^{2}\right)$, then plot the principal rate of strain with the largest absolute value; $0.5 \%$ of the grid points are used and in every case the largest principal rate of strain was negative, or compressive, with stretching in the two directions perpendicular to this component to maintain incompressibility. We might expect the largest principal rate of strain to be compressive because the velocity-derivative skewness (14) is negative. That is, by comparing the skewness with one of its isotropic forms (the trace of the rate of strain cubed, (17a)), we see that a negative skewness implies a negative value for the largest principal rate of strain.

In figures $17(a, b)$ there are obvious structures in the rate-of-strain field, but it is not clear from rotation whether these are tubes or sheets. However, there is definite alignment with respect to the vorticity and scalar gradient which is consistent with the calculated statistics. First, because $F_{2}(33)$, the correlation between the magnitudes of the rate of strain and vorticity, is large, we expect the rate of strain to be concentrated near the vortex tubes. In figure 17(a) the largest strain structure is located near the strong vortex tube in figure 13(a). Next, since there will, on the average, be vortex stretching along the tubes, any compressive components should be perpendicular to the tubes. By comparing figures 13(a) and 17(a) it appears that the compressive component of the rate of strain is aligned perpendicular to the vortex tube. This is further illustrated by comparing figure 18 , which plots a two-dimensional slice of the strain, with figure 15 , the slice of the vorticity. The slice in figure 18 is the same as in figures 15 and 16. Finally, how do the stretching, or expansive, components of the rate of strain align with the vorticity? A linearized model due to Viellefosse (1982) shows the larger expansive component aligned perpendicular to the vorticity, and the smaller expansive component aligried along the vorticity. Since one of the rotationally-invariant forms of the skewness ( $17 \mathrm{~b})$ is related to vortex stretching, and both the skewness and $F_{3}$ are small, our statistics also suggest that the smaller expansive principal rate of strain is aligned with the vorticity. Unfortunately, we have no graphics that show the expansive components of the rate of strain clearly.

How would we expect the scalar gradient to be aligned with respect to the rate of strain? First, the scalar-gradient sheets and rate-of-strain structures should be located near one another because the fourth-order correla- 
tions between the rate of strain and scalar gradient $(44,45)$ are greater than one. Second, the scalar gradient should be aligned along the compressive component of the rate of strain because the mixed-derivative skewness is negative. That is, just as we predicted that the largest component of the rate of strain is compressive by comparing the velocity-derivative skewness with one of its isotropic forms, by comparing the mixed-derivative skewness with its isotropic form (18) we predict that the compressive component of the rate of strain is aligned with the scalar gradient. Comparisons between figures $14 \mathrm{a}, \mathrm{b}$ and 16 to $17 \mathrm{a}, \mathrm{b}$ and 18 show that both of these predictions are borne out.

$\S$ H. Discussion We have studied the derivative correlations of an idealized turbulent flow because they are a quantitative means of probing the small scales of that flow. Numerically, the derivative correlations helped establish the resolution of our simulations. Once the resolution was established, they were compared with experiments and phenomenological models and were used in the interpretation of three-dimensional graphics. Because the small scales of turbulence are believed to be decoupled from the large scales, what is learned about the small scales in this idealized flow should be applicable to turbulent flows in general.

The most important result for the velocity-derivative correlations is that they do not obey the scaling laws predicted by statistical models of intermittency, such as the lognormal model and the $\beta$-model. These models predict that the skewness will depend on Reynolds number and that all the fourthorder velocity-derivative correlations will have the same Reynolds-number dependence. In the simulations the skewness does not depend on the Reynolds number and each of the rotationally-invariant fourth-order correlations has a different exponent. It is possible that at very high Reynolds numbers the velocity-derivative correlations do behave as these models predict and there are high-Reynolds-number experiments (Tavoularis et al. 1978) that show the skewness increasing and a different power law for the velocity-derivative flatness (Van Atta and Antonia 1980). Alternatively, models that include the structure of the small scales, such as the model of Tennekes (1968), might be necessary. The skewness is consistent with the suggestion of Tennekes that vortex stretching is caused by large eddies the size of the Taylor microscale. Tennekes's model also suggests that the velocity-derivative flatness increases with Reynolds number with $\alpha_{4}=1.0$. Both our simulation and experiments find a much smaller value for $\alpha_{4}$. In appendix $B$ it is shown how simple modifications of Tennekes's model can give smaller values of $\alpha_{4}$, while $\alpha_{3}$ 
remains zero. This might provide a basis for a more complete model of smallscale turbulence. A simple experiment which might resolve this issue is to find the exponents of more of the fourth-order correlations.

The most important result for the derivative correlations of a passive scalar is that the scalar-derivative flatness is larger than the velocity-derivative flatness. This suggests that the scalar derivative is more intermittent than the velocity derivative and might be related to several other anomalous scalar effects seen experimentally, for example the "bump" in the scalar spectrum described by Hill (1978) and sharp gradients, or interfaces, called "ramps" (Antonia et al. 1979). What allows sharp structures to form in the scalar field, but not the velocity field? First, they do appear in the velocity field, but as vorticity, which cannot be measured directly, and not as simple velocity gradients, which are easy to measure. These structures are much more likely to be observed in the $\partial u_{2} / \partial x_{1}$ field, than in $\partial u_{1} / \partial x_{1}$, if our conclusions about the relative strengths of $I_{5}$ and $I_{6}(36,37)$ are correct.

Graphical display has been used to demonstrate the alignment indicated by the statistics. This shows that the vorticity is concentrated in tubes, not sheets, with large concentrations of the rate of strain and scalar gradient nearby. The statistics and graphics show that the largest principal rate of strain is compressive and aligned perpendicular to the tube. The larger stretching, or expansive, component of the rate of strain also appears to be perpendicular to the vortex tubes, while the stretching along the tubes is small and is probably not caused by the immediate vortex tube. This structure and the statistics are consistent with the linearized model of Viellefosse (1982) and the structure model of Tennekes (1968), who predicted that the velocityderivative skewness is independent of Reynolds number. Graphical display also shows that large values of the scalar gradient are wound in sheets around the tube and that the gradient is aligned perpendicular to the vorticity and along the compressive component of the rate of strain. This is consistent with correlations between the scalar gradient and both the rate of strain and the vorticity.

Although flow visualization has shown the existence of extended "coherent" structures on the large scales, most theoretical models of the small scales of turbulence have neglected alignment. They assume that small-scale structures in fully-developed turbulence are distributed with random orientation and that statistical mechanics is applicable. Our results suggest that turbulence is characterized by extended vortex tubes and strong alignment between the vorticity and rate of strain. It is possible that as the Reynolds 
number increases these tubes become more tightly wound and the alignment becomes stronger. There would still be statistics, but the statistics would have to include structures. Strong alignment is not inconsistent with the best known characteristic of turbulence, the $k^{-5 / 3}$ spectrum. Lundgren (1983) has shown how fluctuations about a Burger's vortex can give a $k^{-5 / 3}$ spectrum. Our highest Reynolds number simulation also shows a short $-5 / 3$ regime. Even if the correlations behave as the statistical phenomenological models predict at higher Reynolds numbers, there should still be a tendency for the vorticity to form tubes and for there to be alignment between the vorticity and rate of strain. The vortex tubes might show more random orientation than in our current graphics, but there would still be strong alignment at the smallest scales.

Often, subgrid modelling is used with a three-dimensional code (Moin and Kim 1982) to achieve higher Reynolds numbers. This might be useful for studying scalar transport, but would be inappropriate in our work. Subgrid modelling would smooth the flow and result in lower, and incorrect, skewness and flatness factors.

The next step in studying small-scale statistics with numerical codes will be the influence of large-scale shear, strain and temperature gradients. For example, it is known that in the presence of a shear and temperature gradient that the scalar-derivative skewness (16) is nonzero. We also want to learn more about the universality of the isotropic statistics in anisotropic flows and study "band-averaged" statistics. By band-averaged we mean calculating flatnesses based on only part of the velocity spectrum. This has been done experimentally (Tavoularis and Corrsin 1981) and comparisons will be made. After that we will experiment with different subgrid models and see how well they preserve the band-averaged statistics. We hope that once a clear picture of the small-scale structures is developed that improved methods of subgrid modelling can be found.

Future areas of work with passive scalars are the return to isotropy of an anisotropic scalar distribution, the dispersion of an interface, the correlation between two independent scalars, and, eventually, chemical reactions. Turbulent chemical reactions are believed to occur primarily along the sharp interfaces that develop in the scalar field. Intermittency will have a significant effect on the development of these interfaces.

§Acknowledgements I wish to thank E. D. Siggia and R. S. Rogallo for assistance in code development and to thank them, A. Wray and J. R. Herring for many useful discussions. The major portion of this paper was done while I 
was a National Research Council Associate at NASA Ames Research Center. I also acknowledge initial computational support from the National Center for Atmospheric Research and the support of NSF grant ENG-7902942. The National Center for Atmospheric Research is sponsored by the National Science Foundation.

§Appendix A: Errors Many of the trends calculated in this paper, in particular the dependencies on Reynolds and Prandtl numbers, could be sensitive to moderate to large errors in our statistical sample. Therefore, in tables 3-6 the correlations and their statistical variances are given. Where possible, isotropic forms of the correlations have been used to effectively triple the sample size and reduce the errors. For one case, F7b, errors are given based on comparisons with a much longer simulation. By comparing the variances and errors in F7b we can determine a characteristic sample size for each statistic, $N_{s}$, which we define by

$$
(\text { percentage } \text { error })_{s}=\frac{(\text { variance })_{s}}{\sqrt{\left(N_{\delta}-1\right)}} .
$$

To calculate the errors for the other forced simulations, first estimate the sample size by comparing the number of eddy-turnover times with the number in F7b. Then calculate the error by

$$
(\text { percentage error })_{s}=\frac{(\text { variance })_{s}}{\sqrt{N_{s}\left(N_{e} / N_{F 7}\right)-1}}
$$

where $N_{F 7}$ is the number of eddy-turnover times in F7b, $N_{e}$ is the number in F13, and $N_{s}$ is the sample size for the flatness in F7b. As an example, we find the error for the velocity-derivative flatness for F13 (table 3),

$$
2.0 \%=\frac{5.2 \%}{\sqrt{14(2.6 / 4.5)-1}}
$$

Detailed tables of the errors for the $32^{3}$ simulations are given in Kerr (1981). All the third- and fourth-order correlations have error bars which are less than $10 \%$. Fifth- and higher-order correlations and variances were not calculated for F7, but based on the large variances for these statistics in the other 
simulations (tables 5 and 6 ), we expect that their errors are very large. Fifthand higher-order correlations are included only to allow a rough comparison with the phenomenological models. Third- and fourth-order statistics in tables 5 and 6 were calculated without the isotropic forms discussed and as a result their errors are much higher than those shown in tables 3 and 4.

Because of their significance, the time-dependence of the velocity-derivative skewness (14) and one of the fourth-order velocity-derivative correlations, $F_{3}$ (34), are shown for several Reynolds numbers in figures 3(a) and 6(a), respectively. The time-scale has been normalized by the large-scale eddyturnover time, $t_{e}$, for each Reynolds number. These figures demonstrate that the trends discussed in two earlier sections (Spectra and Skewness; Fourthand Higher Order Velocity Correlations) are not statistical errors. Typical times over which samples were taken are indicated by the dashed lines in figures $3(\mathrm{~b})$ and $6(\mathrm{a})$.

§Appendix B: Extension of Tennekes model Let us first review the assumptions made by Tennekes (1968) to find the Reynolds-number dependence of the velocity-derivative skewness and flatness. His first assumption was that the small-scale vortices are strained by vortices whose scale is the Taylor microscale, $\lambda$. This is equivalent to assuming that the small-scale vortices feel only the average rate of strain,

$$
U / \lambda \text {. }
$$

Next, he assumed that the small-scale vortices have a characteristic width, which we will call $\zeta$. He assumed that $\zeta=\eta$, the Kolmogorov microscale, but we will only assume that $\lambda>\varsigma>\eta$. This is equivalent to assuming that it is some sort of average vortex tube that is significant. To find the higher-order correlations, Tennekes assumed that only the vorticity within the small-scale structures matters. This is the average vorticity squared, $(U / \lambda)^{2}$, divided by the volume occupied by the small-scale structures, which if they are tubes of width $\zeta$ and bend over a distance $\lambda$, is

$$
(s / \lambda)^{2}
$$

Therefore, within the tubes the vorticity is

$$
\frac{(U / \lambda)^{2}}{(\zeta / \lambda)^{2}}=\left(\frac{U}{\zeta}\right)^{2}
$$


To find the skewness, Tennekes used (17b) and multiplied the vorticity within the tubes (B3), the average rate of strain (B1), and the volume of the vortices (B2), then normalized by $(U / \lambda)^{3}$. This gives

$$
S_{u} \sim \frac{\langle\omega e \omega\rangle}{(\epsilon / \nu)^{3 / 2}} \sim \frac{(U / \zeta)^{2}(U / \lambda)(\zeta / \lambda)^{2}}{(U / \lambda)^{3}} \sim 1
$$

Notice that all factors of $\zeta$ and $\lambda$ drop out, so it is not critical that the size of the small-scale structures be assumed to be the Kolmogorov microscale.

To find the flatness, one simply squares the vorticity within the small-scale structures, multiplies by their volume, and normalizes by $(U / \lambda)^{4}$. This gives

$$
F_{\partial u^{4}}=\frac{(U / \zeta)^{4}(\zeta / \lambda)^{2}}{\left.(U / \lambda)^{4}\right)} \sim\left(\frac{\zeta}{\lambda}\right)^{2}
$$

If $\zeta=\eta$, then as Tennekes showed, $F_{\partial u^{4}} \sim R_{\lambda}$, or $\alpha_{4}=1$. In our simulations, with $\alpha_{4}<<1$, we require that

$$
\zeta=\lambda \times R_{\lambda}^{\alpha_{4} / 2}
$$

It also follows that when $\mathrm{n}$ is even that $\alpha_{n}=\alpha_{4} \times \frac{n-2}{2}$. This is the same equation, (5), as found by the $\beta$-model of Frisch, Sulem, and Nelkin (1978). When $\mathrm{n}$ is odd our model suggests that $\alpha_{n}=\alpha_{(n-1)}$. As pointed out, our simulations and experiments do not support the $\beta$-model. Nor does this simple model explain why the velocity-derivative flatness and vorticity flatness have different scaling exponents. But a simple model such as this might be the starting point of a more complete model of the small scales. 
§References Antonia, R. A. \& Chambers, A. J. 1980 On the correlation between turbulent velocity and temperature derivatives in the atmospheric surface layer. Boundary Layer Met. 18, 399-410.

Antonia, R. A., Chambers, A. J., Friehe, C. A. \& Van Atta, C. W. 1979 Temperature ramps in the atmospheric surface layer. J. Atmos. Sci. 36, 99-108.

Antonia, R. A., Satyaprakash, B. R., \& Hussain, A. K. M. F. 1982 Statistics of fine-scale velocity in turbulent plane and circular jets. J. Fluid. Mech. 119, 55-89.

Antonia, R. A. \& Van Atta, C. W. 1978 Structure functions of temperature fluctuations in turbulent shear flows. J. Fluid Mech. 84, 561-580.

Arakawa, A. 1962 Computational design for Long-Term Numerical Integration of the Equations of Fluid Motion: Two-Dimensional Incompressible Flow. Part 1. J. Comp. Phys. 1, 119-143.

Aref, H. \& Siggia, E. D. 1980 Vortex dynamics of the two-dimensional turbulent shear layer. J. Fluid Mech. 100, 705-738.

Batchelor, G. K. 1952 The effect of homogeneous turbulence on material lines and surfaces. Proc. Roy. Soc. A 213, 349-366.

Batchelor, G. K. 1959 Small-scale variation of convected quantities like temperature in turbulent fluid. Part 1. General discussion and the case of small conductivity. J. Fluid Mech. 5, 113-133.

Batchelor, G. K., Howells, I. D. \& Townsend, A. A. 1959 Small-scale variation of convected quantities like temperature in turbulent fluid. Part 2. The case of large conductivity. J. Fluid Mech. 5, 134-139.

Betchov, R. 1956 An inequality concerning the production of vorticity in isotropic turbulence. J. Fluid Mech. 1, 497-504.

Brown, G. L. \& Roshko, A. 1974 On density effects and large structures in turbulent mixing layers. J. Fluid Mech. 64, 775-816.

Champagne, F. H., Friehe, C. A., LaRue, J. C., \& Wyngaard, J. C. 1977 Flux measurements, flux estimation techniques and fine-scale turbulence measurements in the unstable surface layer over land. J. Atmos. Sci. 34, 515.

Clay, J. P. 1973 Turbulent mixing of temperature in water, air and mercury. Ph.D. thesis, University of California at San Diego.

Corrsin, S. 1951 On the spectrum of isotropic temperature fluctuations in isotropic turbulence. J. Appl. Phys. 22, 469-473.

Corrsin, S. 1962 Turbulent dissipation fluctuations. Phys. Fluids 5, 13011302.

Frenkiel, F. M. \& Klebanoff, P. S. 1975 On the lognormality of the small- 
scale structure of turbulence. Boundary Layer Met. 8, 173.

Frisch, U., Sulem, P. L. \& Nelkin, M. 1979 A simple model of intermittent fully-developed turbulence. J. Fluid Mech. 87, 719-736.

Gibson, C. H., Stegen, G. R., \& Williams, R. B. 1970 Statistics of the fine structure of turbulent velocity and temperature fields measured at high Reynolds number. J. Fluid Mech. 41153.

Gibson, C. H. 1968 Fine structure of scalar fields mixed by turbulence. II. Spectral theory. Phys. Fluids 11, 2316-2327.

Herring, J. R. \& Kerr, R. M. 1982 Comparison of direct numerical simulations with predictions of two-point closures for isotropic turbulence convecting a passive scalar. J. Fluid Mech. 118, 205-219.

Hill, R. J. 1978 Models of the scalar spectrum for turbulent advection. J. Fluid Mech. 88, 541-562.

Kerr, R. M. 1981 Theoretical investigation of a passive scalar such as temperature in isotropic turbulence. Ph.D. thesis; Cooperative Thesis no. 64, Cornell University and National Center for Atmospheric Research.

Klebanoff, P. S. 1982 National Bureau of Standards, private communication.

Kolmogorov, A. N. 1941 Local structure of turbulence in an incompressible fluid at very high Reynolds numbers. C. R. Acad. Sci. UUSR 30, 301-305.

Kolmogorov, A. N. 1962 A refinement of previous hypotheses concerning the local structure of turbulence in a viscous incompressible fluid at high Reynolds number. J. Fluid Mech. 13, 82-85.

Kuo, A. Y. \& Corrsin, S. 1971 Experiments on internal intermittency and fine-structure distribution functions in fully turbulent fluid. J. Fluid Mech. $50,285-319$.

Larcheveque, M., Chollet, J. P., Herring, J. R., Lesieur, M., Newman, G. R. \& Schertzer, D. 1980 Two-point closure applied to a passive scalar in decaying isotropic turbulence. In Turbulent Shear Flows 2 (eds. J. S. Bradbury, F. Durst, B. E. Launder, F. W. Schmidt \& J. H. Whitelaw), pp. 50-60. Springer.

Lundgren, T. S. 1983 Strained spiral vortex model for turbulent fine structure. Phys. Fluids 26, 2193-2203.

Moin P. \& Kim J. 1982 Numerical investigation of turbulent channel flow. J. Fluid Mech. 118, 341-377.

Monin, A. S. \& Yaglom, A. M. 1975 Statistical Fluid Mechanics, vol. 2. Massachusetts Institute of Technology Press.

Nelkin, M. 1981 Do the dissipation fluctuations in high-Reynolds-number 
turbulence define a universal exponent? Phys. Fluids 24, 556.

Nelkin, M. \& Bell, T. L. 1978 One-exponent scaling for very high-Reynoldsnumber turbulence. Phys. Rev. A 17, 363-369.

Orszag, S. A. 1971 Numerical simulation of incompressible flows within simple boundaries: accuracy. J. Fluid Mech. 49, 75-112.

Orszag, S. A. \& Patterson, G. S. 1972 Numerical simulation of turbulence. In Statistical Models and Turbulence (eds. M. Rosenblatt \& C. Van Atta), Lecture Notes in Physics, vol. 12, pp. 127-147. Springer.

Pao, Y-H. 1965 Structure of turbulent velocity and scalar fields at large wavenumbers. Phys. Fluids 8, 1063-1075.

Park, J. T. 1976 Inertial subrange turbulence measurements in the marine boundary layer. $\mathrm{Ph} . \mathrm{D}$. thesis, University of California, San Diego.

Patterson, G. S. 1980 National Center for Atmospheric Research, private communication.

Patterson, G. S. \& Orszag, S. A. 1971 Spectral calculations of isotropic turbulence: efficient removal of aliasing interactions. Phys. Fluids 14, 25382541.

Rogallo, R. S. 1981 Numerical experiments in homogeneous turbulence. NASA TM 81315.

Saffman, P. G. 1968 Lectures in homogeneous turbulence. in Topics in Nonlinear Physics (ed. N. Zabusky), 485-614. Springer.

Siggia, E. D. 1981a Numerical study of small scale intermittency in three dimensional turbulence. J. Fluid Mech. 107, 375-406.

Siggia, E. D. 1981b Invariants for the one-point vorticity and strain rate correlation functions. Phys. Fluids 24, 1934-1936.

Siggia, E. D. \& Patterson, G. S. 1978 Intermittency effects in a numerical simulation of stationary three-dimensional turbulence. J. Fluid Mech. 86, 567-592.

Sreenivasan, K. R. \& Tavoularis, S. 1980 On the skewness of the temperature derivative in turbulent flows. J. Fluid Mech. 101, 783-795.

Sreenivasan, K. R., Tavoularis, S., Henry, R. \& Corrsin, S. 1980 Temperature fluctuations and scales in grid-generated turbulence. J. Fluid Mech. 100, 597-621.

Tavoularis, S., Bennett, S. C. \& Corrsin, S. 1978 Velocity-derivative skewness in small Reynolds number, nearly isotropic turbulence. J. Fluid Mech. 88, 63-69.

Tavoularis, S. \& Corrsin, S. 1981 Experiments in nearly homogeneous turbulent shear flow with a uniform mean temperature gradient. Part 2. 
The fine structure. J. Fluid Mech. 104, 349-367.

Tennekes, H. 1968 Simple model for the small-scale structure of turbulence. Phys. Fluids 11, 669-671.

Van Atta, C. W. 1974 Influence of fluctuations on dissipation rates of some statistical properties of turbulent scalar fields. Izv. Atmos. Ocean Phys. 10, 712-719.

Van Atta, C. W. \& Antonia, R. A. 1980 Reynolds-number dependence of skewness and flatness factors of turbulent velocity derivatives. Phys. Fluids $23,252-257$.

Viellefosse, P. 1982 Local interaction between vorticity and shear in a perfect incompressible fluid. J. de Physique 43, 837-842.

Warhaft, Z. \& Lumley, J. L. 1978 An experimental study of the decay of temperature fluctuation in grid-generated turbulence. J. Fluid Mech. 88, 659-684.

Winant, C. D. \& Browand, F. K. 1974 Vortex pairing: the mechanism of turbulent mixing layer growth at moderate Reynolds number. J. Fluid Mech. 63, 237-255.

Wray, A. 1981 Very low storage time-advancement schemes. NASA Ames Research Center, private communication.

Wyngaard, J.C. 1971 The effect of velocity sensitivity on temperature derivative statistics in isotropic turbulence. J. Fluid Mech. 48, 763-769. 
TABLE 1.- CHARACTERISTICS OF FORCED SIMULATIONS

\begin{tabular}{|l|r|r|r|r|r|r|r|l|}
\hline Fun & Mesh & \multicolumn{1}{|c|}{$\mathrm{R}_{\lambda}$} & $\mathrm{Pr}$ & \multicolumn{1}{|c|}{$\Delta \mathrm{t}^{a}$} & \multicolumn{1}{c|}{$\mathrm{N}_{\mathrm{f}}^{b}$} & \multicolumn{1}{c|}{$\mathrm{K}_{\mathrm{k}}$} & \multicolumn{1}{|c|}{$\mathrm{K}_{\mathrm{OC}}$} & $\mathrm{t}_{\mathrm{e}}$ \\
\hline F1 & $32^{3}$ & 9.0 & 2.0 & 19.0 & 20 & 9.4 & 15.8 & 4.7 \\
F2 & $32^{3}$ & 9.0 & .5 & 19.0 & 20 & 9.4 & 5.6 & 4.7 \\
F3 & $32^{3}$ & 12.3 & 2.0 & 13.6 & 35 & 11.9 & 20.0 & 3.55 \\
F4 & $32^{3}$ & 12.3 & 1.0 & 13.6 & 35 & 11.9 & 11.9 & 3.55 \\
F5 & $32^{3}$ & 12.3 & .5 & 13.6 & 35 & 11.9 & 7.8 & 3.55 \\
F6 & $32^{3}$ & 18.5 & 1.0 & 9.6 & 25 & 16.5 & 16.5 & 1.97 \\
F7a & $32^{3}$ & 18.5 & .5 & 39.0 & 40 & 16.4 & 9.8 & 1.98 \\
F7b & $32^{3}$ & 18.5 & .5 & 9.0 & 10 & 16.5 & 9.8 & 1.97 \\
F8 & $32^{3}$ & 18.5 & .1 & 9.6 & 25 & 16.5 & 2.9 & 1.97 \\
F9 & $32^{3}$ & 24.0 & .5 & 9.5 & 20 & 21.4 & 12.7 & 1.26 \\
F10 & $32^{3}$ & 24.0 & .1 & 9.5 & 20 & 21.4 & 3.8 & 1.26 \\
F11 & $32^{3}$ & 28.9 & .5 & 29.5 & 60 & 25.7 & 15.3 & .91 \\
F12 & $32^{3}$ & 28.9 & -- & 29.5 & 60 & 25.8 & -- & .91 \\
F13 & $64^{3}$ & 28.5 & 1.0 & 10.0 & 11 & 12.3 & 12.3 & 3.83 \\
F14 & $64^{3}$ & 28.5 & .5 & 10.0 & 11 & 12.3 & 7.3 & 3.83 \\
F15 & $64^{3}$ & 28.5 & .1 & 10.0 & 11 & 12.3 & 2.2 & 3.83 \\
F16 & $64^{3}$ & 37.5 & 1.0 & 4.5 & 10 & 16.0 & 16.0 & 2.51 \\
F17 & $64^{3}$ & 37.5 & .5 & 4.5 & 10 & 16.0 & 9.5 & 2.51 \\
F18 & $64^{3}$ & 37.5 & .1 & 4.5 & 10 & 16.0 & 2.8 & 2.51 \\
F19 & $64^{3}$ & 48.2 & 1.0 & 6.75 & 28 & 22.4 & 22.4 & 1.41 \\
F20 & $64^{3}$ & 48.2 & .5 & 6.75 & 28 & 22.4 & 13.3 & 1.41 \\
F21 & $64^{3}$ & 48.2 & .1 & 6.75 & 28 & 22.4 & 4.0 & 1.41 \\
F22 & $64^{3}$ & 55.9 & 1.0 & 4.00 & 17 & 27.2 & 27.2 & 1.05 \\
F23 & $64^{3}$ & 55.9 & .5 & 4.00 & 17 & 27.2 & 16.2 & 1.05 \\
F24 & $64^{3}$ & 55.9 & .1 & 4.00 & 17 & 27.2 & 4.8 & 1.05 \\
F25 & $128^{3}$ & 82.9 & 1.0 & 1.35 & 27 & 45.7 & 45.7 & .85 \\
F26 & $128^{3}$ & 82.9 & .5 & 1.35 & 27 & 45.7 & 27.2 & .85 \\
F27 & $128^{3}$ & 82.9 & .1 & 1.35 & 27 & 45.7 & 8.1 & .85 \\
\hline
\end{tabular}

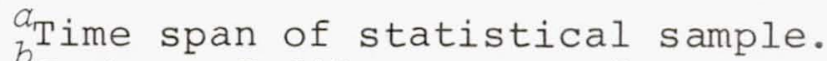
Number of files averaged. 
TABLE 2.- VELOCITY AND SCALAR FLATNESS FACTORS USED TO DETERMINE ALIASING ERRORS

\begin{tabular}{|l|c|c|c|c|c|c|}
\hline Run & Grid size & $\operatorname{Pr}$ & $F_{\partial u^{4}}(64)$ & $F_{\partial \theta^{4}}(64)$ & $F_{\partial u^{4}}(32)$ & $F_{\partial \theta^{4}}(32)$ \\
\hline \multicolumn{7}{|c|}{ Averages of many realizations } \\
\hline F11 & 32 & 0.5 & -- & -- & 3.94 & 5.73 \\
F12 & 32 & -- & -- & -- & 4.17 & -- \\
F13 & 64 & 1.0 & 4.18 & 6.88 & -- & -- \\
F14 & 64 & .5 & 4.18 & 5.82 & -- & -- \\
F15 & 64 & .1 & 4.18 & 3.61 & -- & - \\
\hline \multicolumn{7}{|c|}{ One realization } \\
\hline F13 & 64 & 1.0 & 2.99 & 7.30 & 3.93 & 5.62 \\
F14 & 64 & .5 & 3.99 & 5.95 & 3.93 & 5.08 \\
F15 & 64 & .1 & 3.99 & 3.55 & 3.93 & 3.57 \\
\hline
\end{tabular}

Note: F11 is $32^{3}$ aliased. F12 is $32^{3}$ de-aliased. The size of the grid used to calculate the flatness is in parentheses.

TABLE 3.- VELOCITY DERIVATIVE SKEWNESS AND FLATNESS FACTORS AND VARIANCES

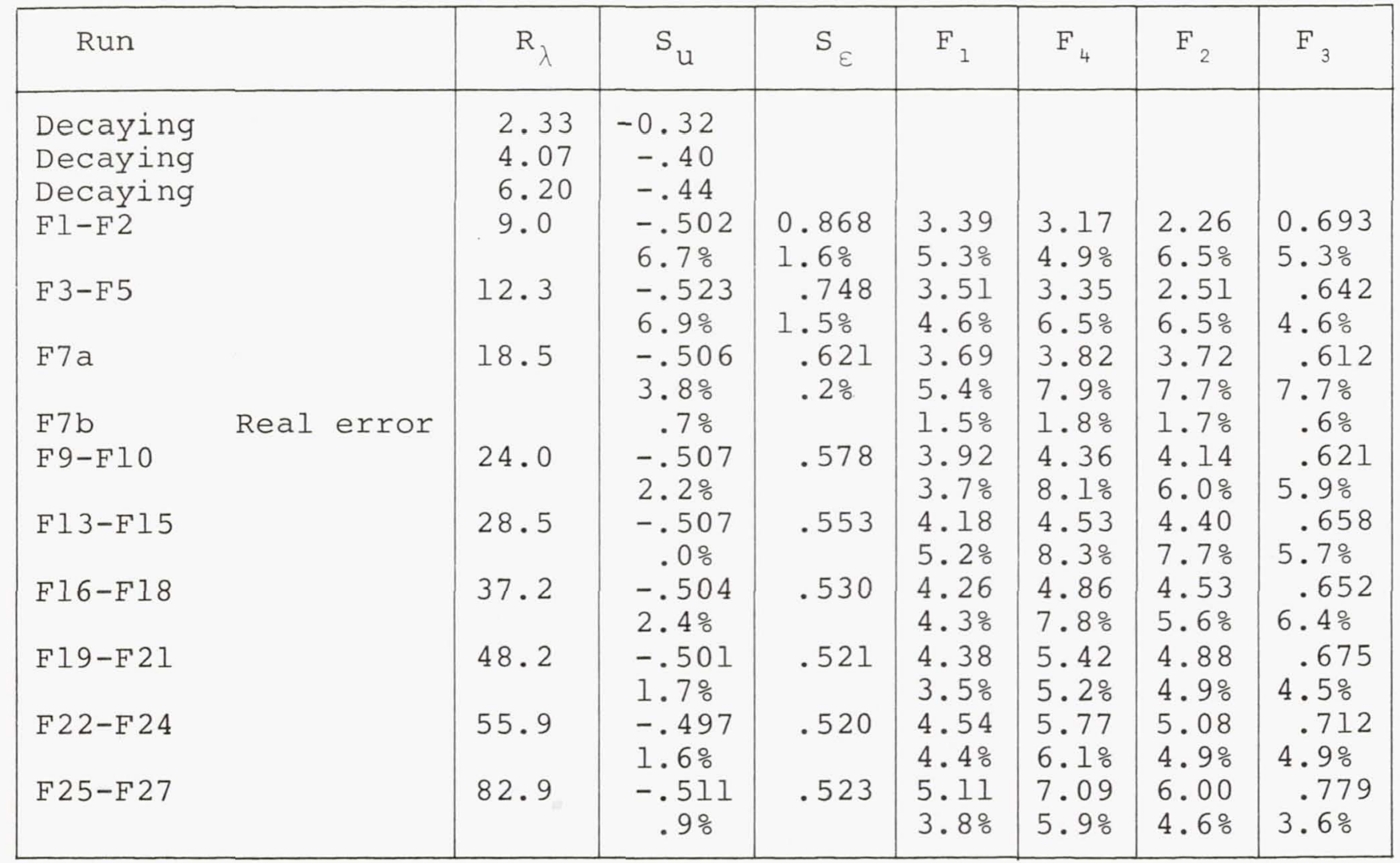


TABLE 4.- SCALAR- AND MIXED-DERIVATIVE SKEWNESS AND FLATNESS FACTORS AND VARIANCES

\begin{tabular}{|c|c|c|c|c|c|c|c|c|c|}
\hline Run & $\mathrm{R}_{\lambda}$ & Pr & $\mathrm{S}_{\mathrm{u} \theta}$ & $\mathrm{s}_{x}$ & $\mathrm{~F}_{\nabla \theta}$ & $\mathrm{F}_{\nabla \theta \omega 1}$ & $\mathrm{~F}_{\nabla \theta \mathrm{e} I}$ & $\mathrm{~F}_{\nabla \theta \omega 2}$ & $\mathrm{~F}_{\nabla \theta \mathrm{e}_{2}}$ \\
\hline \multirow[t]{2}{*}{ Fl } & 9.0 & 2.0 & -0.523 & 0.585 & 5.18 & 0.94 & 1.16 & 0.663 & 1.77 \\
\hline & & & $3.8 \%$ & & $14.3 \%$ & $5.1 \%$ & $6.1 \%$ & $10.4 \%$ & $7.9 \%$ \\
\hline \multirow[t]{2}{*}{ F 2} & 9.0 & .5 & -.420 & .749 & 3.74 & .97 & 1.07 & .768 & 1.49 \\
\hline & & & $3.3 \%$ & & $5.8 \%$ & $4.6 \%$ & $4.4 \%$ & $11.7 \%$ & $6.4 \%$ \\
\hline \multirow[t]{2}{*}{ F3 } & 12.3 & 2.0 & -.520 & .554 & 5.83 & .93 & 1.18 & .636 & 1.82 \\
\hline & & & $3.8 \%$ & & $11.4 \%$ & $5.6 \%$ & $6.4 \%$ & $8.8 \%$ & $8.0 \%$ \\
\hline F 4 & 12.3 & 1.0 & $\begin{array}{l}-.510 \\
3.9 \%\end{array}$ & .608 & $\begin{array}{r}5.11 \\
11.2 \%\end{array}$ & $\begin{array}{r}.93 \\
6.5 \%\end{array}$ & $\begin{array}{l}1.16 \\
6.8 \%\end{array}$ & $\begin{array}{l}.657 \\
8.0 \%\end{array}$ & $\begin{array}{l}1.76 \\
8.5 \%\end{array}$ \\
\hline \multirow[t]{2}{*}{ F5 } & 12.3 & .5 & -.465 & .673 & 4.25 & .95 & 1.12 & .708 & 1.64 \\
\hline & & & $4.3 \%$ & & $6.9 \%$ & $5.7 \%$ & $5.8 \%$ & $8.3 \%$ & $7.5 \%$ \\
\hline \multirow[t]{2}{*}{$\mathrm{F} 6$} & 18.5 & 1.0 & -.506 & .551 & 5.87 & .92 & 1.19 & .624 & 1.82 \\
\hline & & & $4.9 \%$ & & $9.4 \%$ & $5.3 \%$ & $6.0 \%$ & $7.5 \%$ & $7.1 \%$ \\
\hline \multirow[t]{2}{*}{ F7a } & 18.5 & .5 & -.479 & .579 & 4.93 & .94 & 1.12 & .690 & 1.69 \\
\hline & & & $3.1 \%$ & & $7.2 \%$ & $8.3 \%$ & $4.0 \%$ & $8.8 \%$ & $4.0 \%$ \\
\hline F7b & Real & errors & $\pm .6 \%$ & & $-2.7 \%$ & $+2.0 \%$ & $+2.8 \%$ & $\pm .9 \%$ & $+2.8 \%$ \\
\hline \multirow{2}{*}{ F 8} & 18.5 & .1 & -.286 & .813 & 3.37 & .98 & 1.03 & .843 & $1.3 ?$ \\
\hline & & & $8.7 \%$ & & $2.9 \%$ & $4.9 \%$ & $4.9 \%$ & $5.4 \%$ & $5.3 \%$ \\
\hline \multirow{2}{*}{ F9 } & 24.0 & .5 & -.486 & .549 & 5.63 & .94 & 1.18 & .663 & 1.81 \\
\hline & & & $4.1 \%$ & & $12.2 \%$ & $5.1 \%$ & $5.3 \%$ & $7.9 \%$ & $5.8 \%$ \\
\hline \multirow{2}{*}{ F10 } & 24.0 & .1 & -.344 & .657 & 3.58 & .98 & 1.05 & .801 & 1.43 \\
\hline & Г & & $7.3 \%$ & & $6.0 \%$ & $3.9 \%$ & $4.0 \%$ & $5.6 \%$ & $4.6 \%$ \\
\hline F13 & 28.5 & 1.0 & -.517 & .527 & 6.88 & 1.03 & 1.38 & .217 & 2.14 \\
\hline \multirow{2}{*}{ F14 } & 20 & & $3.0 \%$ & & & $7.2 \%$ & $5.8 \%$ & $9.6 \%$ & $6.2 \%$ \\
\hline & 20.0 & .3 & -.506 & .544 & 5.82 & 1.02 & 1.33 & .281 & 2.05 \\
\hline \multirow{2}{*}{ F15 } & 285 & 7 & 267 & & 30 & - & & $9.9 \%$ & $4.9 \%$ \\
\hline & & $\cdot 1$ & $5.7 \%$ & . & $10 \quad 2 \%$ & $7.3 \%$ & $\begin{array}{l}1.13 \\
4.2 \%\end{array}$ & 6.351 & 1.56 \\
\hline \multirow[t]{2}{*}{ F16 } & 37.2 & 1.0 & -.521 & .523 & 7.57 & 1.06 & 1.41 & .154 & 2.21 \\
\hline & & & $2.5 \%$ & & $7.5 \%$ & $6.6 \%$ & $5.6 \%$ & $8.9 \%$ & $5.7 \%$ \\
\hline \multirow[t]{2}{*}{ F17 } & 37.2 & .5 & -.517 & .535 & 6.49 & 1.06 & 1.39 & .205 & 2.17 \\
\hline & & & $2.7 \%$ & & $6.3 \%$ & $6.1 \%$ & $4.9 \%$ & $8.8 \%$ & $4.9 \%$ \\
\hline \multirow[t]{2}{*}{ F18 } & 37.2 & .1 & -.408 & .549 & 3.90 & 1.06 & 1.20 & .464 & 1.73 \\
\hline & & & $3.7 \%$ & & $7.1 \%$ & $5.0 \%$ & $4.0 \%$ & $6.6 \%$ & $3.8 \%$ \\
\hline \multirow[t]{2}{*}{ F19 } & 48.5 & 1.0 & -.497 & .511 & 8.12 & 1.02 & 1.36 & .117 & 2.11 \\
\hline & & & $2.6 \%$ & & $9.2 \%$ & $3.7 \%$ & $3.9 \%$ & $5.2 \%$ & $4.3 \%$ \\
\hline \multirow[t]{2}{*}{$\mathrm{F} 20$} & 48.5 & .5 & -.515 & .532 & 7.73 & 1.02 & 1.38 & .139 & 2.15 \\
\hline & & & $3.1 \%$ & & $10.7 \%$ & $3.8 \%$ & $4.2 \%$ & $7.1 \%$ & $4.6 \%$ \\
\hline \multirow[t]{2}{*}{ F21 } & 48.5 & .1 & -.446 & .519 & 5.00 & 1.03 & 1.26 & .352 & 1.89 \\
\hline & & & $4.3 \%$ & & $13.5 \%$ & $3.6 \%$ & $4.1 \%$ & $7.2 \%$ & $4.8 \%$ \\
\hline F22 & 55.9 & 1.0 & -.463 & .493 & 7.98 & 1.02 & 1.33 & .146 & 2.03 \\
\hline & & & $3.1 \%$ & & $11.0 \%$ & $3.5 \%$ & 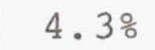 & $7.4 \%$ & $4.6 \%$ \\
\hline F23 & 55.9 & .5 & -.506 & .526 & 8.29 & 1.01 & 1.38 & .127 & 2.14 \\
\hline & & & $3.2 \%$ & & $12.7 \%$ & $3.7 \%$ & $4.5 \%$ & $5.0 \%$ & $4.9 \%$ \\
\hline F24 & 55.9 & .1 & -.457 & .507 & 5.59 & 1.03 & 1.28 & .292 & 1.93 \\
\hline & & & $3.9 \%$ & & $12.2 \%$ & $4.0 \%$ & & $6.0 \%$ & $4.4 \%$ \\
\hline F25 & 82.9 & 1.0 & -.493 & .507 & 10.76 & 1.04 & 1.43 & .076 & 2.23 \\
\hline & & & $2.5 \%$ & & $10.0 \%$ & $4.3 \%$ & $3.9 \%$ & $3.6 \%$ & $4.2 \%$ \\
\hline F26 & 82.9 & .5 & -.516 & .522 & 10.75 & 1.04 & 1.47 & .073 & 2.31 \\
\hline & & & $2.4 \%$ & & $10.7 \%$ & $4.7 \%$ & $4.2 \%$ & $2.9 \%$ & $4.3 \%$ \\
\hline F 27 & 82.9 & .1 & -.473 & .490 & 7.44 & 1.08 & 1.38 & .192 & 2.11 \\
\hline & & & $2.6 \%$ & & $13.2 \%$ & $3.9 \%$ & $4.4 \%$ & $5.6 \%$ & $4.8 \%$ \\
\hline
\end{tabular}


TABLE 5.- HIGHER-ORDER VELOCITY DERIVATIVE AND VORTICITY CORRELATIONS AND VARIANCES

\begin{tabular}{|c|c|c|c|c|c|c|c|c|c|c|}
\hline Run & $\mathrm{R}_{\lambda}$ & $\mathrm{s}_{\mathrm{u}}$ & $\mathrm{F}_{\partial u^{4}}$ & $\mathrm{~F}_{\partial \mathrm{u}^{5}}$ & $F_{\partial u^{6}}$ & $\mathrm{~F}_{\partial \mathrm{u}^{7}}$ & $F_{\partial u^{8}}$ & $\mathrm{~F}_{\omega^{4}}$ & $\mathrm{~F}_{\omega}{ }^{6}$ & $\mathrm{~F}_{\omega^{8}}$ \\
\hline F13-15 & 28.5 & 0.527 & 4.12 & -6.86 & 39.5 & -118 & 701 & 4.69 & 51.8 & 988 \\
\hline & & $24 \%$ & $11 \%$ & $40 \%$ & $41 \%$ & $82 \%$ & $93 \%$ & $13.4 \%$ & $40 \%$ & $70 \%$ \\
\hline \multirow[t]{2}{*}{ F16-18 } & 37.2 & .531 & 4.22 & -6.98 & 40.1 & -117 & 638 & 5.00 & 61.2 & 1302 \\
\hline & & $21 \%$ & $7.8 \%$ & $28 \%$ & $24 \%$ & $45 \%$ & $55 \%$ & $9.1 \%$ & $24 ㅇ ㅡ ㅇ$ & $39 \%$ \\
\hline \multirow[t]{2}{*}{ F19-21 } & 48.5 & .525 & 4.38 & -7.61 & 47.2 & -159 & 1003 & 5.49 & 82.3 & 2620 \\
\hline & & $20 \%$ & $7.7 \%$ & $32 \%$ & $30 \%$ & $83 \%$ & $=76 \%$ & $7.7 \%$ & $35 \%$ & $88 \%$ \\
\hline \multirow[t]{2}{*}{ F 22-24 } & 55.9 & .528 & 4.63 & -8.07 & 53.8 & -179 & 1206 & 5.85 & 96.7 & 3284 \\
\hline & & $17 \%$ & $7.4 \%$ & $25 \%$ & $25 \%$ & $50 \%$ & $62 \%$ & $8.7 \%$ & $33 \%$ & $76 \%$ \\
\hline \multirow[t]{2}{*}{ F $25-27$} & 82.9 & .526 & 5.11 & -9.38 & 73.3 & -281 & 2326 & 7.12 & 185.0 & 15613 \\
\hline & & $10 \%$ & $5.2 \%$ & $20 \%$ & $48 \%$ & $47 \%$ & $56 \%$ & $6.9 \%$ & $48 \%$ & $142 \%$ \\
\hline
\end{tabular}


TABLE 6. - HIGHER-ORDER SCALARDERIVATIVE CORRELATIONS AND VARIANCES

\begin{tabular}{|c|c|c|c|c|c|}
\hline Run & $\mathrm{R}_{\lambda}$ & Pr & $\mathrm{F}_{\partial \theta^{4}}$ & $F_{\partial \theta^{6}}$ & $F_{\partial \theta^{8}}$ \\
\hline F15 & 28.5 & 0.1 & $\begin{array}{c}3.71 \\
25 \%\end{array}$ & $\begin{array}{l}30 \\
67 \%\end{array}$ & $\begin{array}{l}457 \\
103 \%\end{array}$ \\
\hline F18 & 37.2 & .1 & $\begin{array}{l}3.87 \\
13 \%\end{array}$ & $\begin{array}{l}31 \\
33 \%\end{array}$ & $\begin{array}{l}379 \\
55 \%\end{array}$ \\
\hline F 21 & 48.5 & .1 & $\begin{array}{l}4.50 \\
22 \%\end{array}$ & $\begin{array}{l}46 \\
70 \%\end{array}$ & $\begin{array}{l}784 \\
140 \%\end{array}$ \\
\hline F 24 & 55.9 & .1 & $\begin{array}{l}5.68 \\
25 \%\end{array}$ & $\begin{array}{l}89 \\
75 \%\end{array}$ & $\begin{array}{l}2696 \\
127 \%\end{array}$ \\
\hline F 27 & 82.9 & .1 & $\begin{array}{l}7.32 \\
17 \%\end{array}$ & $\begin{array}{r}161 \\
48 \%\end{array}$ & $\begin{array}{r}6932 \\
87 \%\end{array}$ \\
\hline F14 & 28.5 & .5 & $\begin{array}{l}5.87 \\
20 \%\end{array}$ & $\begin{array}{l}89 \\
51 \%\end{array}$ & $\begin{array}{r}2323 \\
82 \%\end{array}$ \\
\hline F17 & 37.2 & .5 & $\begin{array}{l}6.54 \\
13 \%\end{array}$ & $\begin{array}{r}112 \\
33 \%\end{array}$ & $\begin{array}{r}3222 \\
56 \%\end{array}$ \\
\hline F 20 & 48.5 & .5 & $\begin{array}{l}7.29 \\
16 \%\end{array}$ & $\begin{array}{r}144 \\
47 \%\end{array}$ & $\begin{array}{r}4928 \\
84 \%\end{array}$ \\
\hline F 23 & 55.9 & .5 & $\begin{array}{l}8.34 \\
18 \%\end{array}$ & $\begin{array}{l}204 \\
55 \%\end{array}$ & $\begin{array}{l}9300 \\
100 \%\end{array}$ \\
\hline F 26 & 82.9 & .5 & $\begin{array}{l}10.8 \\
14 \%\end{array}$ & $\begin{array}{l}371 \\
39 \%\end{array}$ & $\begin{array}{r}24385 \\
66 \%\end{array}$ \\
\hline F13 & 28.5 & 1.0 & $\begin{array}{l}6.94 \\
17 \%\end{array}$ & $\begin{array}{l}130 \\
43 \%\end{array}$ & $\begin{array}{r}4139 \\
72 \%\end{array}$ \\
\hline $\mathrm{F} 16$ & 37.2 & 1.0 & $\begin{array}{c}7.68 \\
15 \%\end{array}$ & $\begin{array}{l}160 \\
37 \%\end{array}$ & $\begin{array}{r}5669 \\
62 \%\end{array}$ \\
\hline F19 & 48.5 & 1.0 & $\begin{array}{l}7.95 \\
14 \%\end{array}$ & $\begin{array}{c}174 \\
41 \%\end{array}$ & $\begin{array}{r}6772 \\
74 \%\end{array}$ \\
\hline F 22 & 55.9 & 1.0 & $\begin{array}{l}8.02 \\
15 \%\end{array}$ & $\begin{array}{r}183 \\
45 \%\end{array}$ & $\begin{array}{r}7586 \\
85 \%\end{array}$ \\
\hline F 25 & 82.9 & 1.0 & $\begin{array}{l}10.8 \\
13 \%\end{array}$ & $\begin{array}{l}368 \\
38 \%\end{array}$ & $\begin{array}{r}23398 \\
67 \%\end{array}$ \\
\hline
\end{tabular}




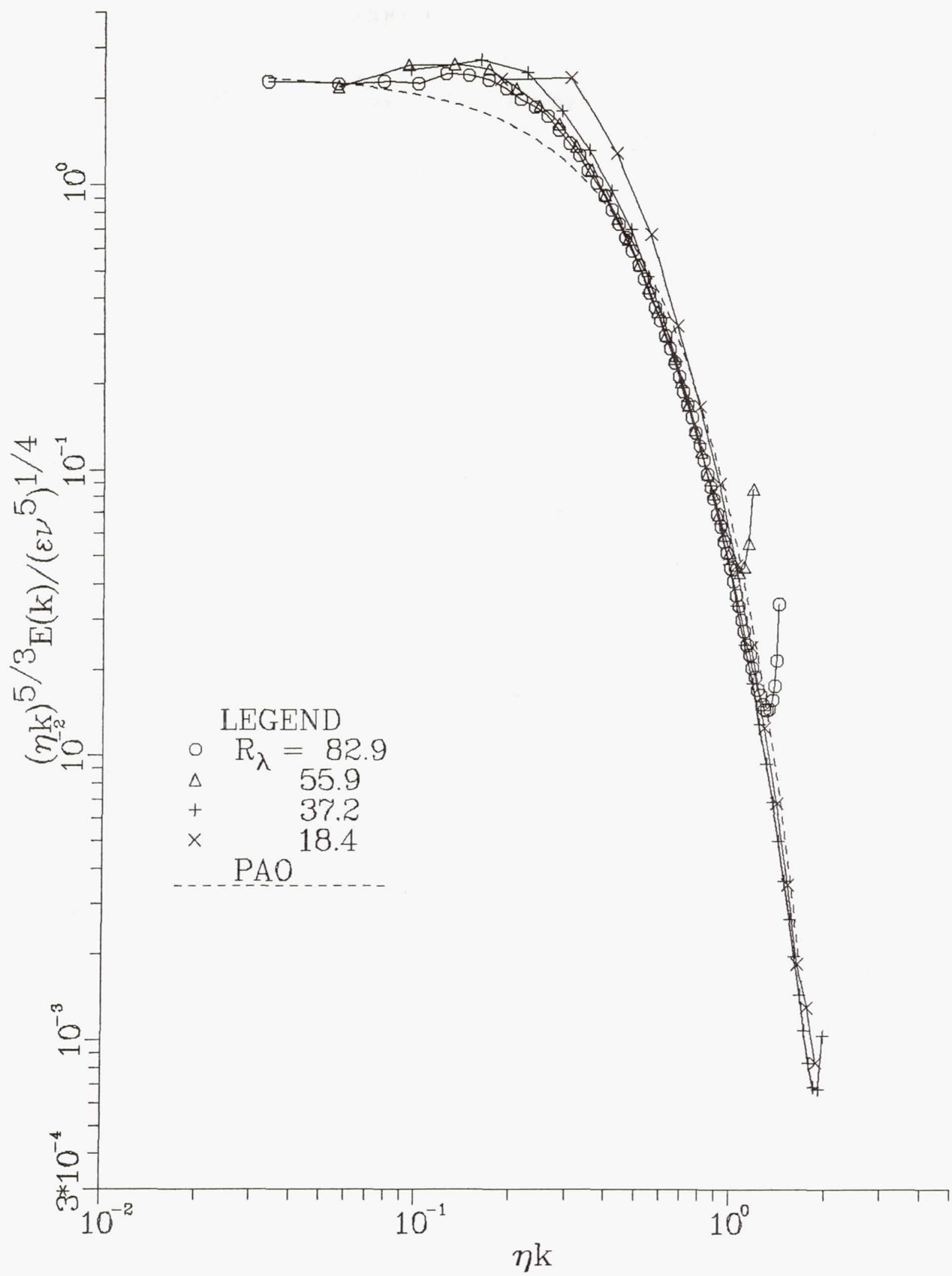

Figure 1.- Kinetic-energy spectra normalized by the Kolmogorov microscales (10a) and multiplied by $k^{5 / 3}$. Pao's theoretical curve (13) for $K_{0}=2.45$ is indicated by the dashed line. Circle: $R_{\lambda}=82.9$. Triangle: $R_{\lambda}=55.9$. Plus: $R_{\lambda}=37.2$. Cross: $R_{\lambda}=18.4$. 


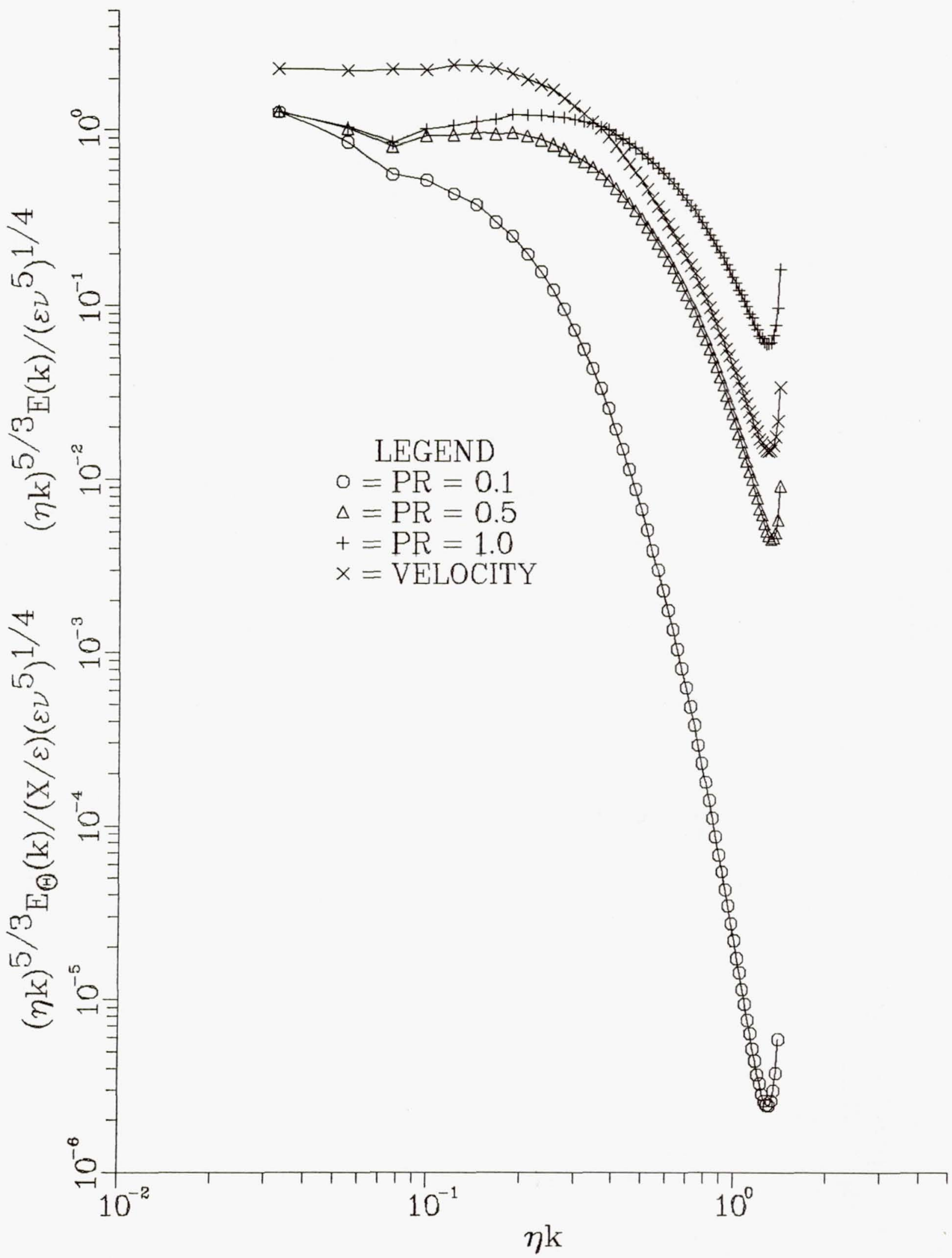

Figure 2. - Kinetic-energy and passive-scalar spectra at $R_{\lambda}=82.9$ normalized by the Kolmogorov microscales (10a) and the scalar-variance dissipation, $\chi$. Circle: $\operatorname{Pr}=0.1$. Triangle: $\operatorname{Pr}=0.5$. Plus: $\operatorname{Pr}=1.0$. Cross: Kinetic energy. 


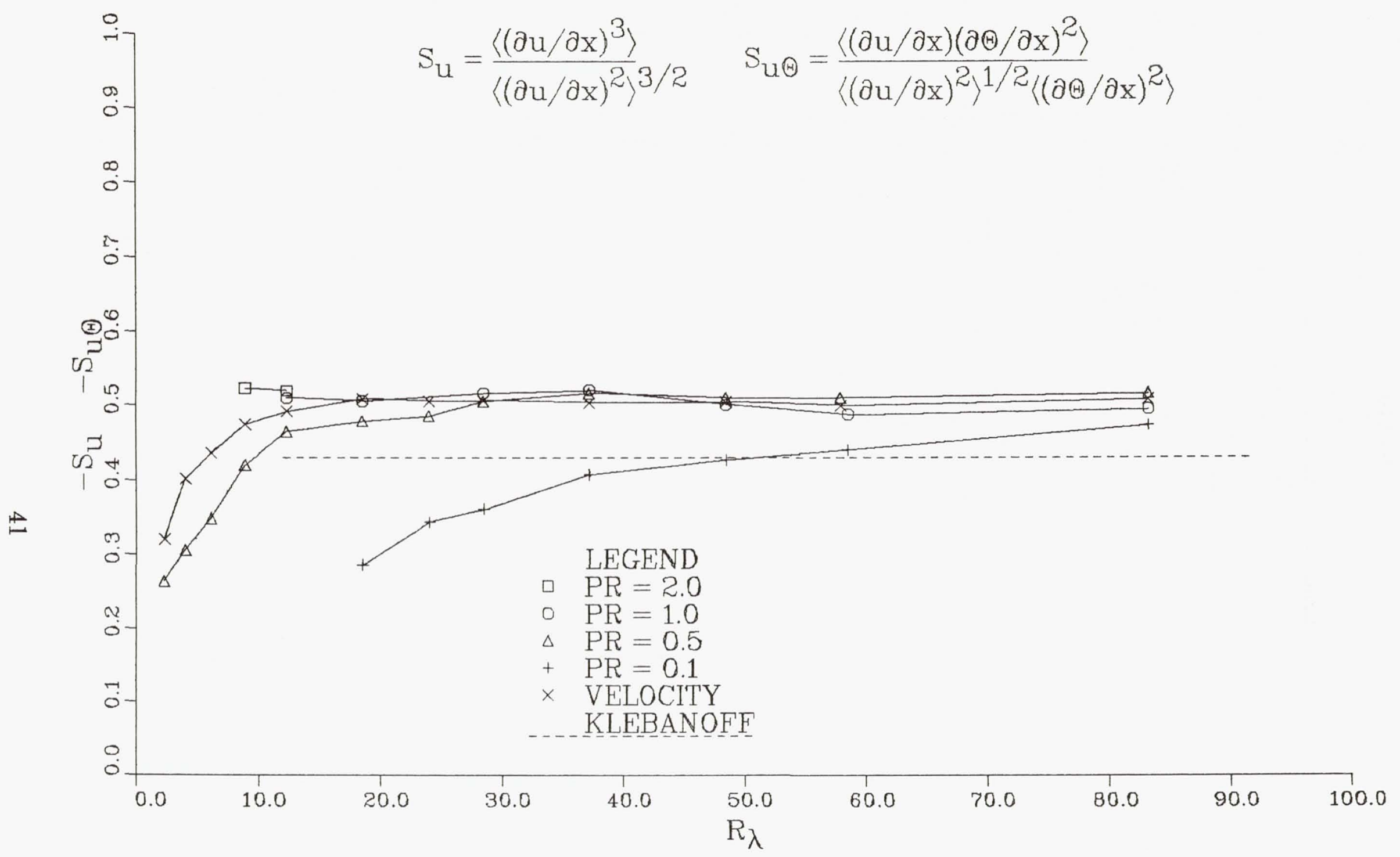

(a) Dependence of the velocity-derivative skewness (14) and mixed-derivative skewness (15) on Taylor-microscale Reynolds number. Dashed line is the experimental data of Klebanoff (private communication) where $S_{u}=0.43$. Square: $\operatorname{Pr}=2.0$. Circle: $\operatorname{Pr}=1.0$. Triangle: $\operatorname{Pr}=0.5$. Plus: $\operatorname{Pr}=0.1$. Cross: Kinetic energy .

Figure 3.- Velocity and mixed-derivative skewnesses. 


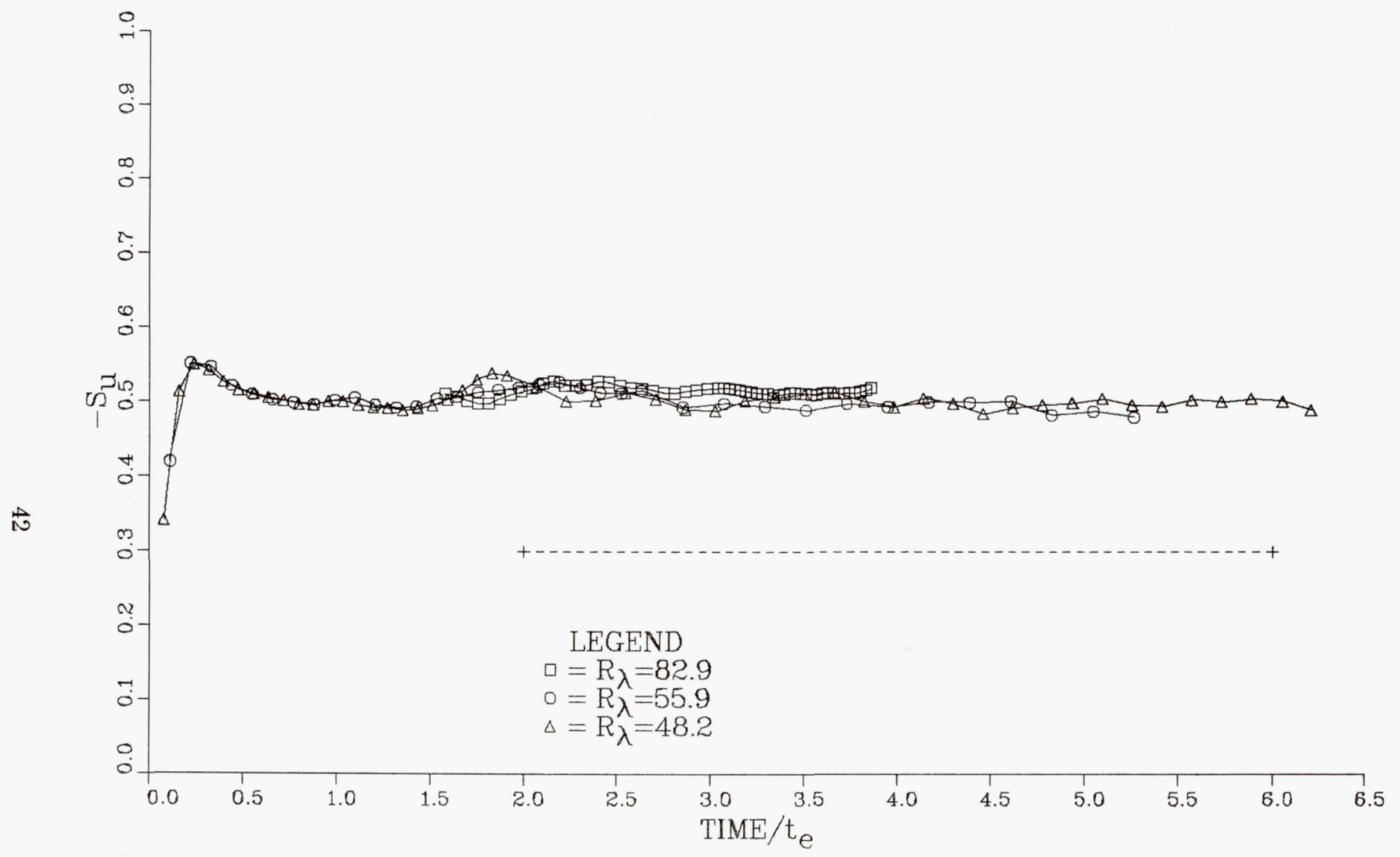

(b) Time evolution of the velocity-derivative skewness (14) for three Reynolds numbers. Time has been normalized by the eddy-turnover time of each simulation. Square: $R_{\lambda}=82.9$. Circle: $R_{\lambda}=55.9$. Triangle: $R_{\lambda}=48.2$. Dashed line represents the time-span over which the ensemble average was taken.

Figure 3.- Concluded. 


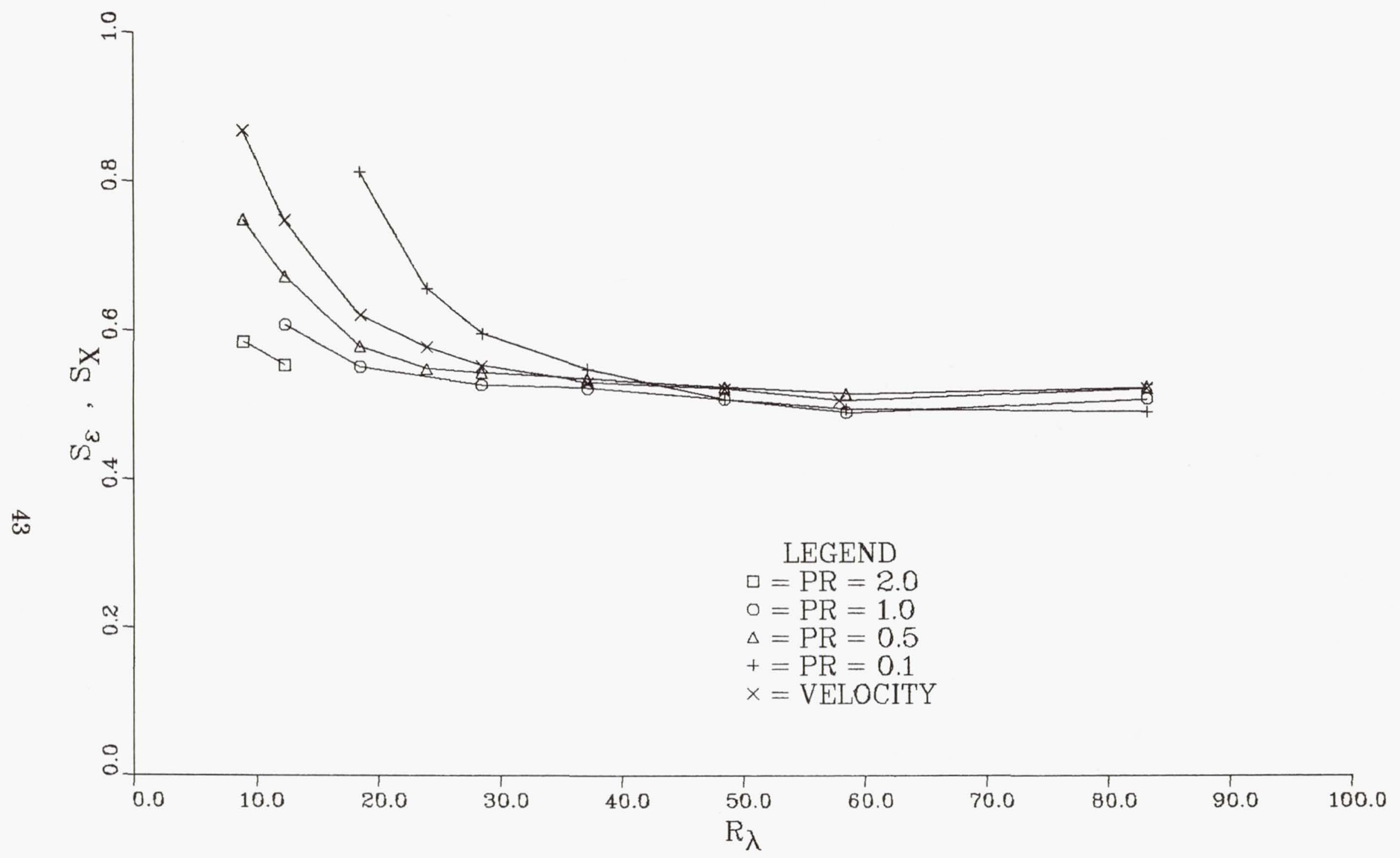

Figure 4.- Dependence of the velocity-dissipation skewness (24) and the scalar-dissipation skewness (25) on Taylor-microscale Reynolds number. 


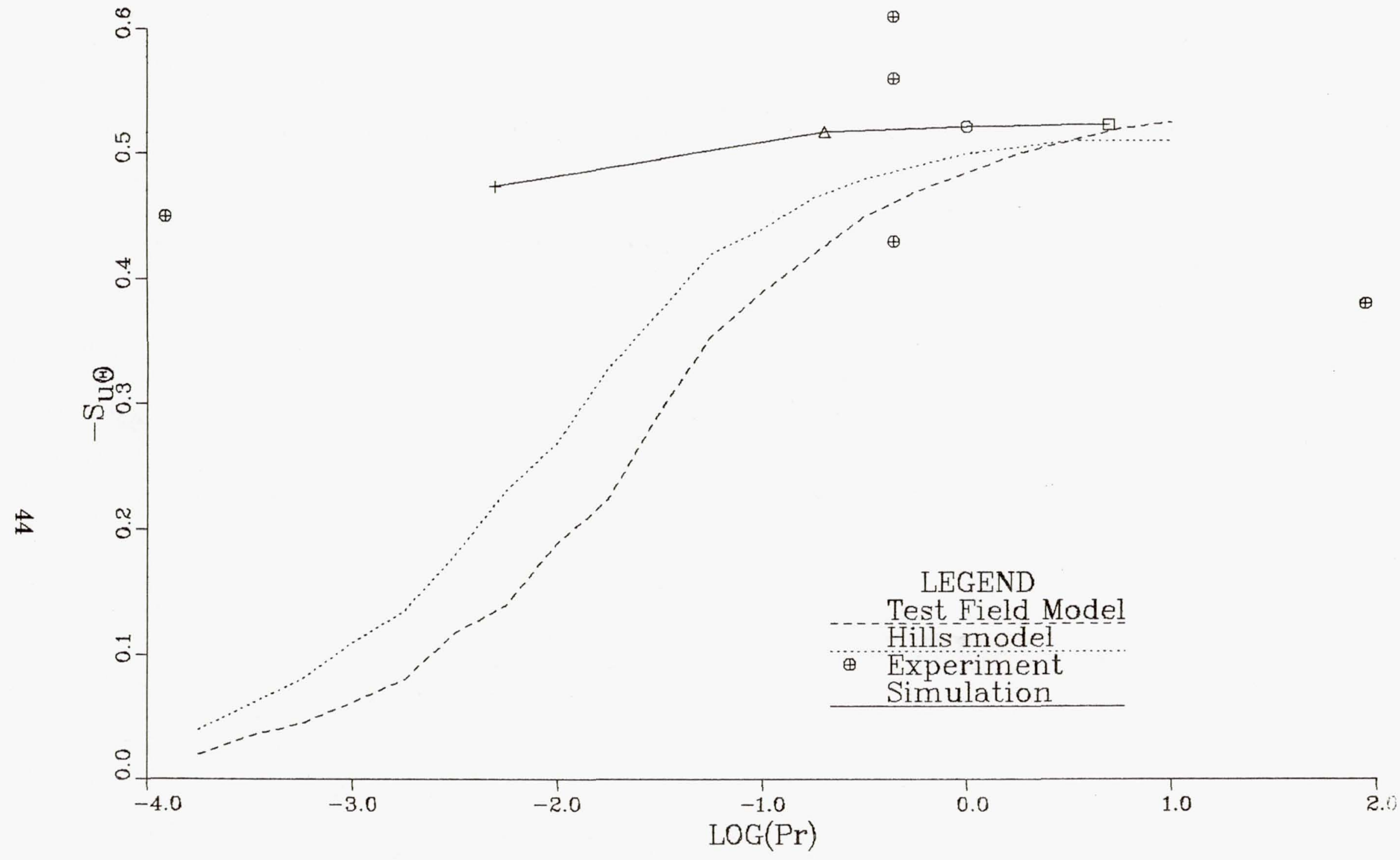

Figure 5.- Dependence of the mixed-derivative skewness (15) on Prandtl number. Test field model results (dashed line), the model of Hill (1978) (dotted line), and experiments (crossed circles) are all taken from Larcheveque et al. (1980). Symbols for our simulations (solid line) are the same as in figure $3 \mathrm{a}$. 


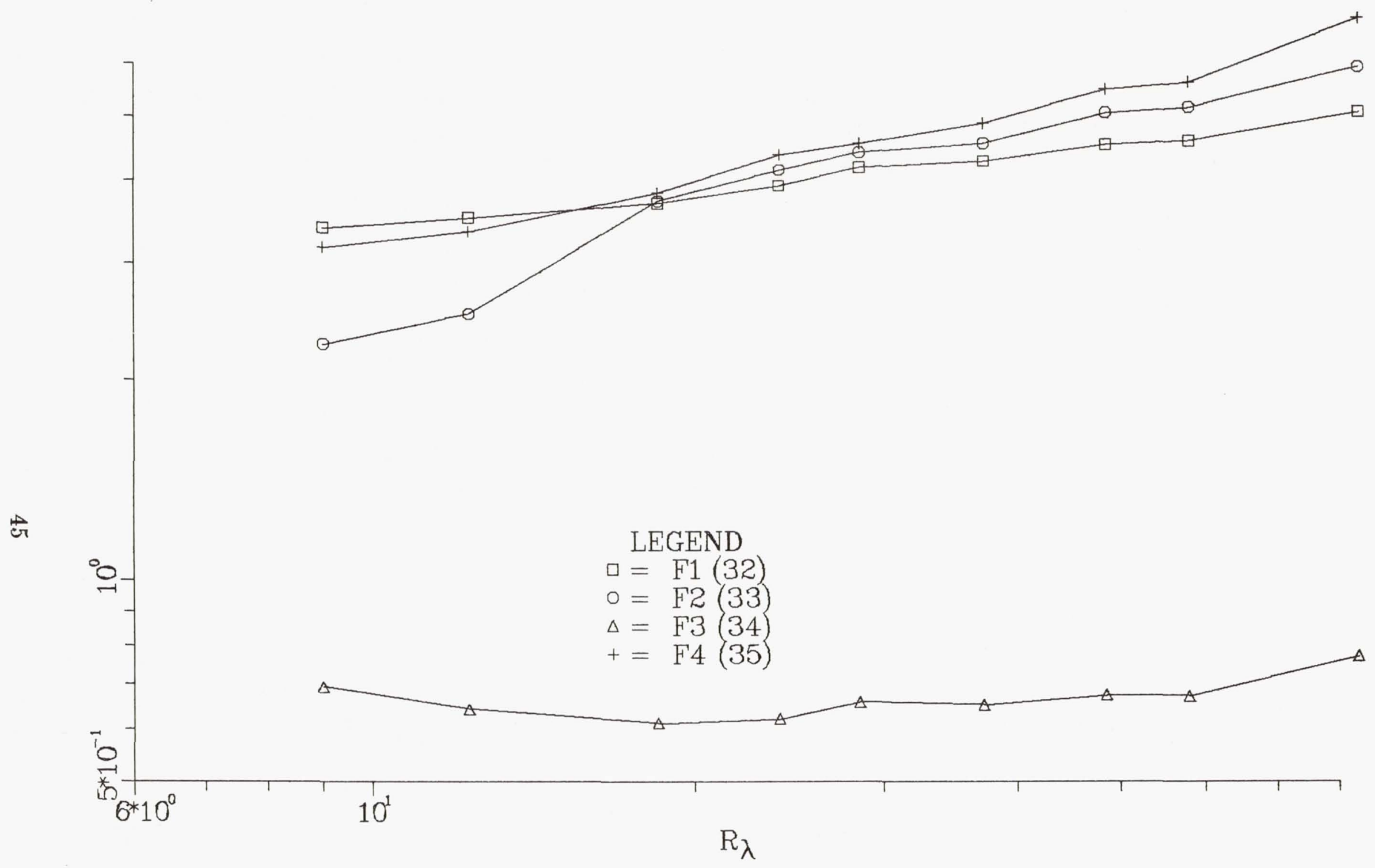

(a) Dependence on Taylor-microscale Reynolds number. Squares are $F_{1}(32)$ or the velocity-derivative flatness, $F_{\partial u^{4}}(3)$. Circles are $F_{2}$ (33). Triangles are $F_{3}(34)$. Pluses are $F_{4}$ or the vorticity flatness, $F_{\omega^{4}}(38)$.

Figure 6.- Rotationally invariant fourth-order velocity derivative correlations. 


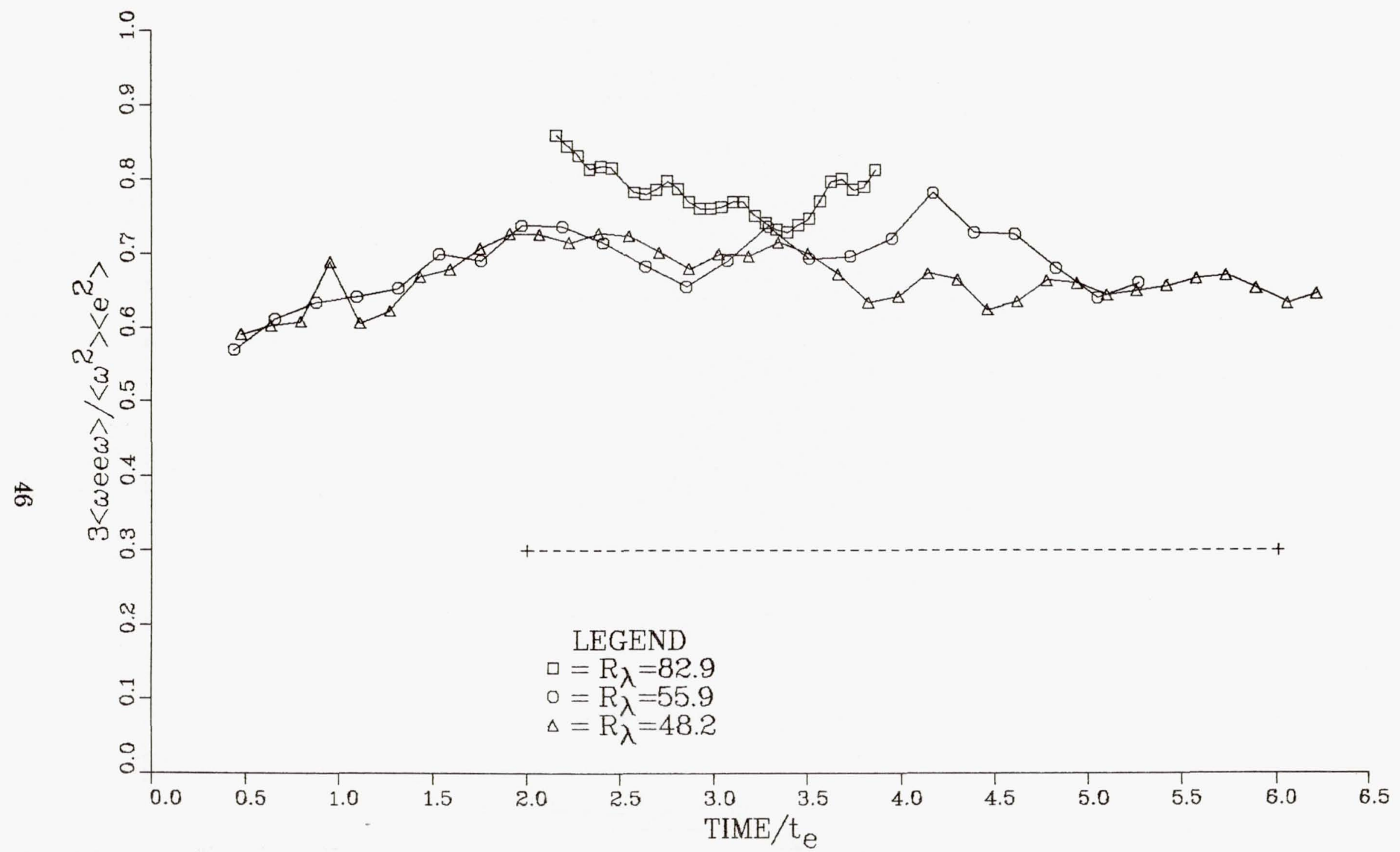

(b) Time evolution of $F_{3}$ (34), for three Reynolds numbers. Time has been normalized by the eddy-turnover time of each simulation. Square: $R_{\lambda}=82.9$. Circle: $R_{\lambda}=55.9$. Triangle: $R_{\lambda}=48.2$. Dashed line represents the time-span over which the ensemble average was taken.

Figure 6.-Concluded. 


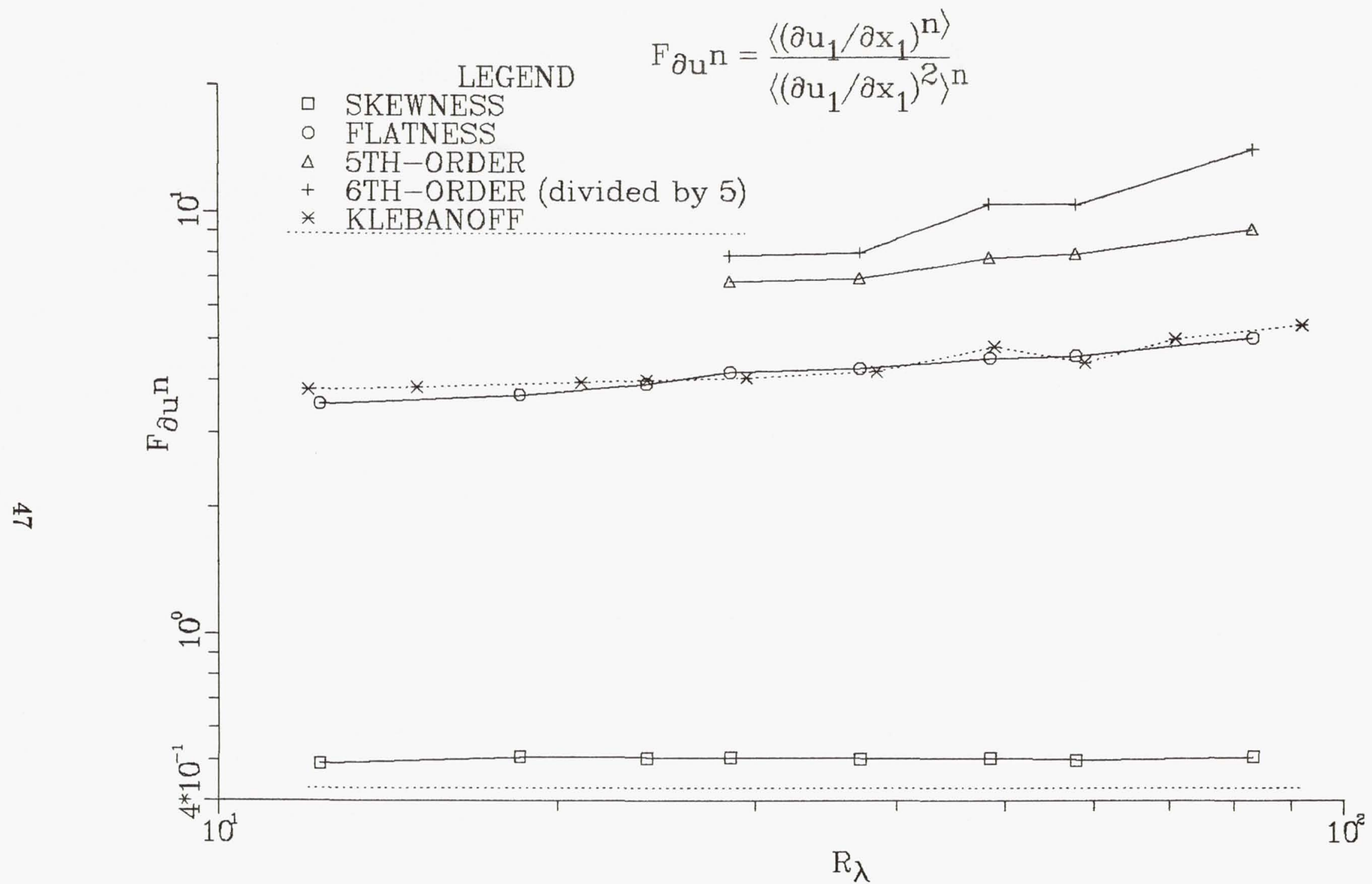

Figure 7.- Dependence of the velocity-derivative correlations, $F_{\partial u n}(3)$ on Taylor-microscale Reynolds number. Squares are third order, or the velocity-derivative skewness, $S_{u}$ (14). Circles are fourth order, the velocity-derivative flatness or $F_{1}(32)$. Triangles are fifth order. Pluses are sixth order, which has been divided by 5 so it will appear on this graph. The dotted lines are experimental data of Klebanoff (private communication) for the velocity-derivative skewness and the velocity-derivative flatness (stars). 


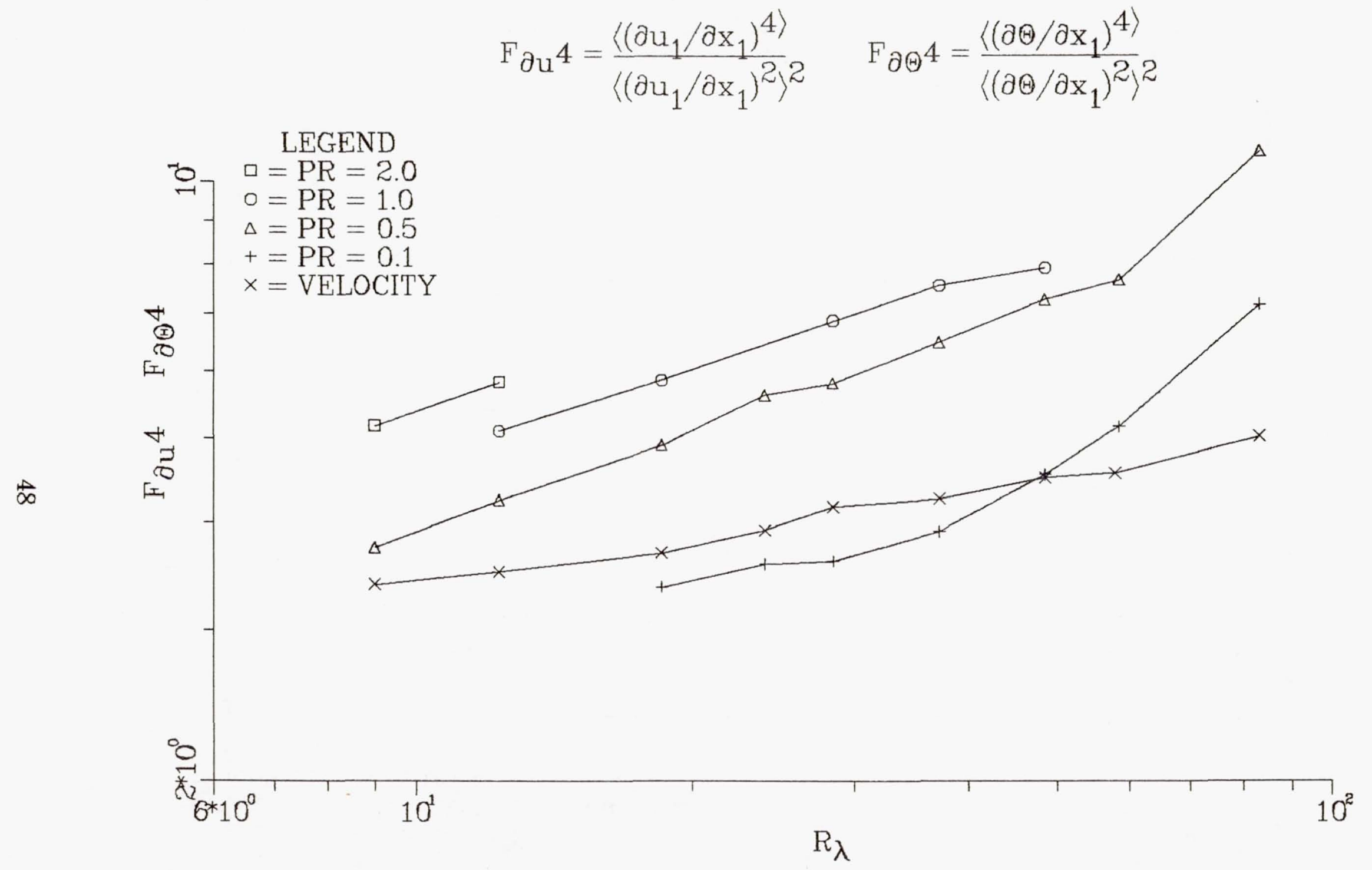

Figure 8.- Dependence of the velocity-derivative flatness $(3,32)$ and the scalar-derivative flatness $(39.41)$ on Taylor-microscale Reynolds number. The experiment of Sreenvasan et al. 1980 is a star. 


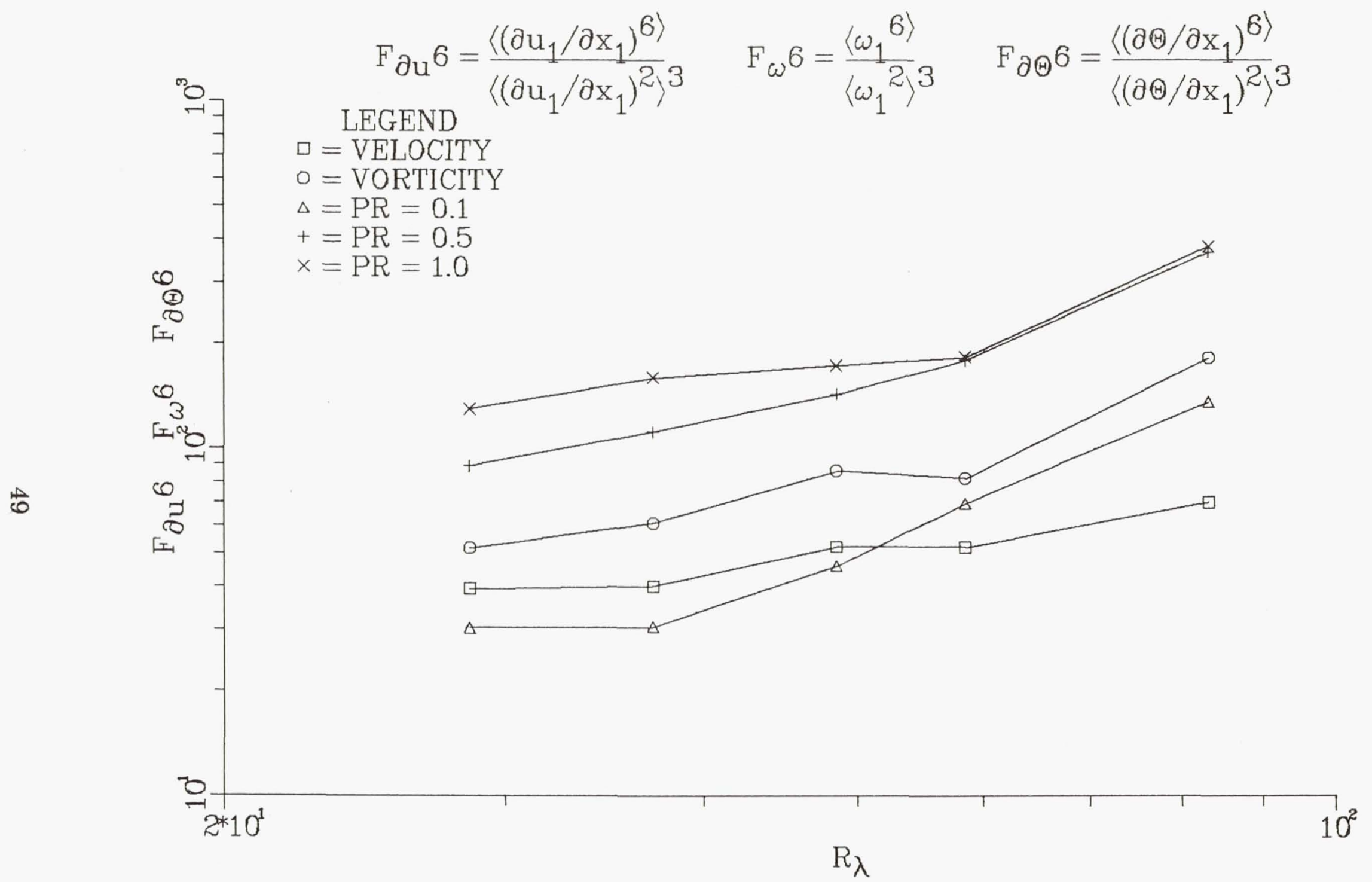

Figure 9.- Dependence of the sixth-order velocity-derivative correlation, vorticity correlation, and scalar-derivative correlations on Taylormicroscale Reynolds number. Squares are the velocity, $F_{\partial u^{6}}(3)$. Circles are the vorticity, $F_{\omega^{6}}(38)$. Triangles are the scalar derivative correlation, $F_{\partial \theta^{6}}$ (39), for $\operatorname{Pr}=0.1$. Pluses are for $\operatorname{Pr}=0.5$. Crosses are for $\operatorname{Pr}=1.0$. 


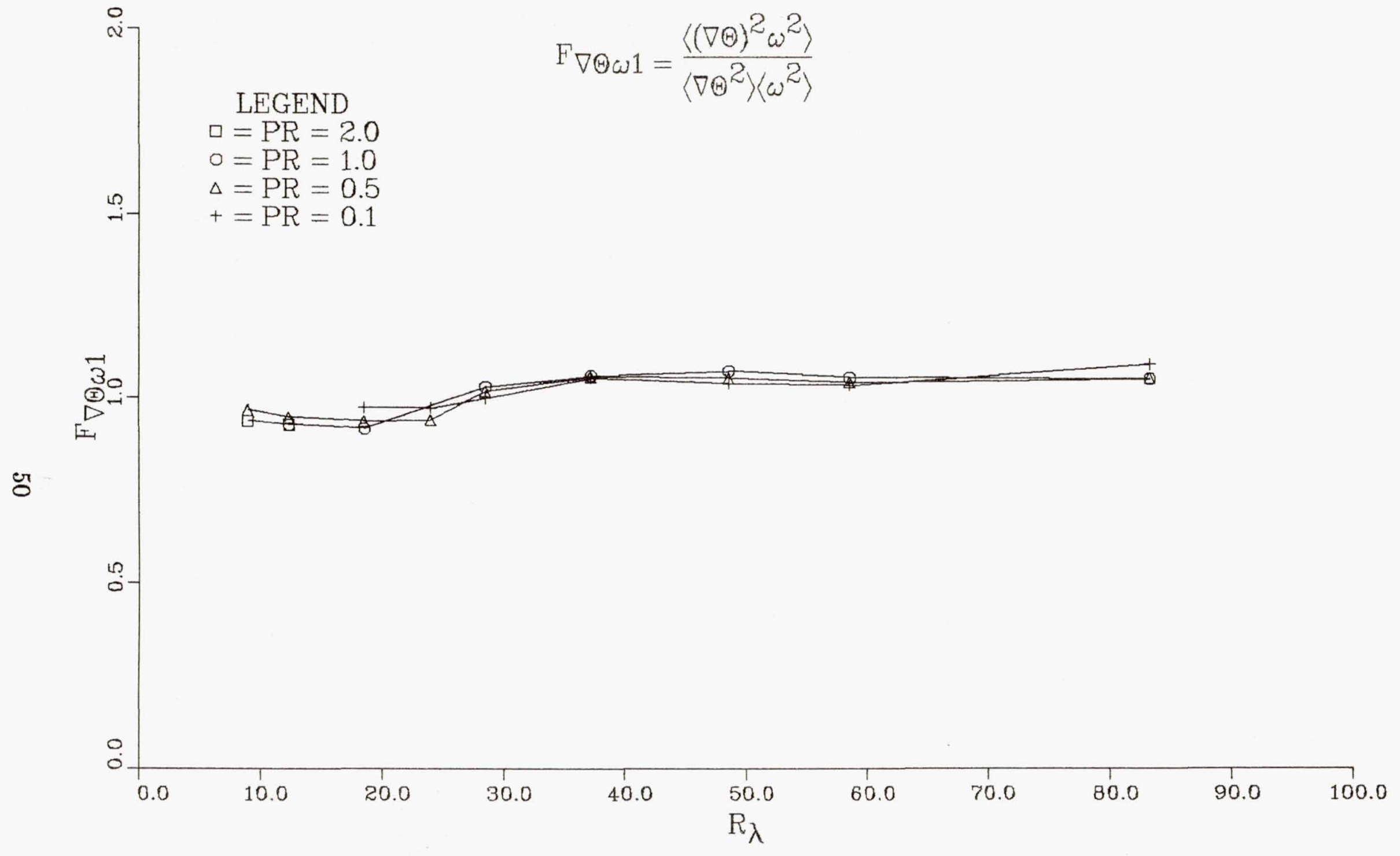

Figure 10.- Dependence of the first scalar derivative-vorticity correlation, $F \nabla \theta \omega_{1}$ (42), on Taylor-microscale Reynolds number. 


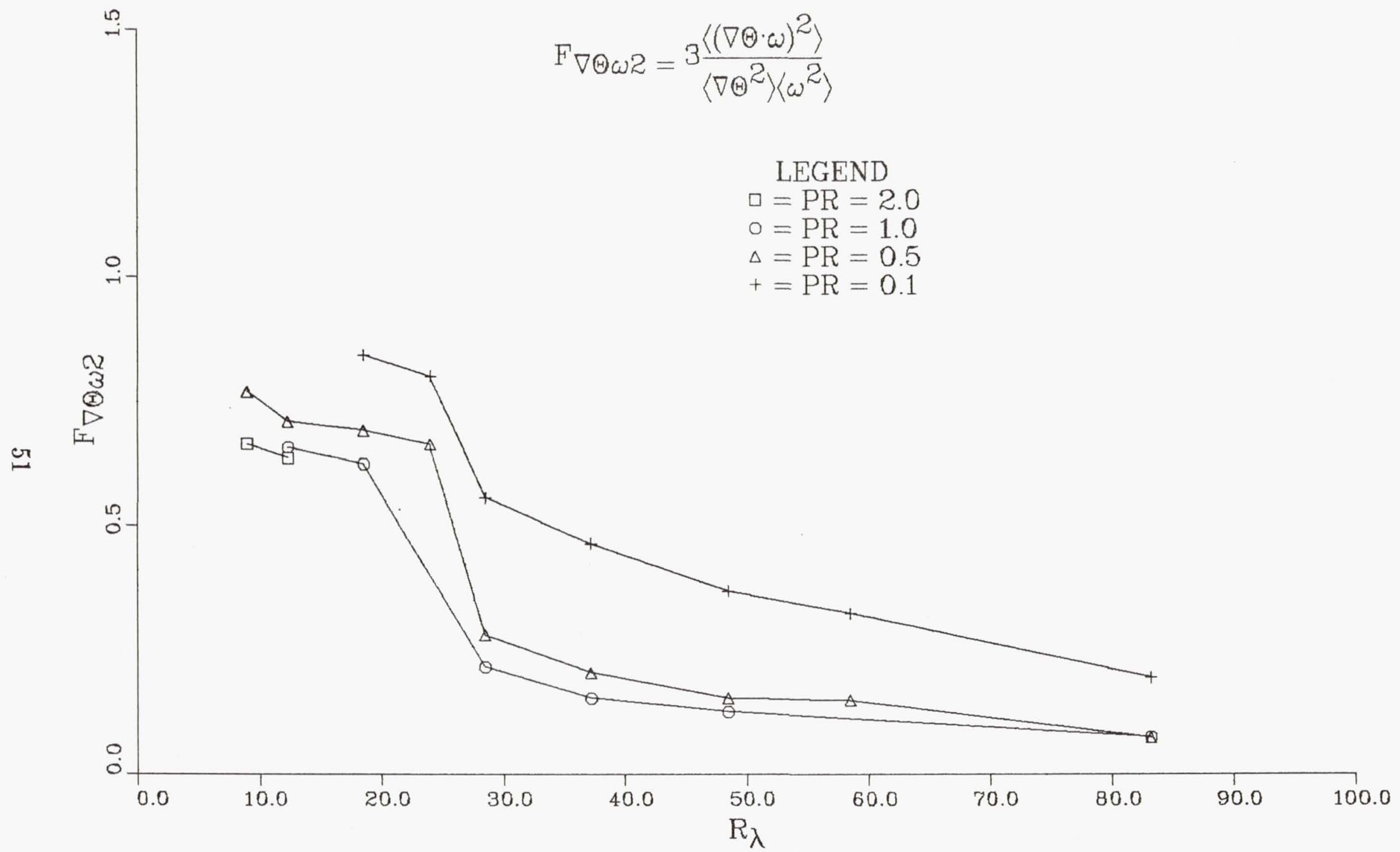

Figure 11.- Dependence of the second scalar derivative-vorticity correlation, $F_{\nabla \theta \omega_{2}}$ (43), on Taylor-microscale Reynolds number. 


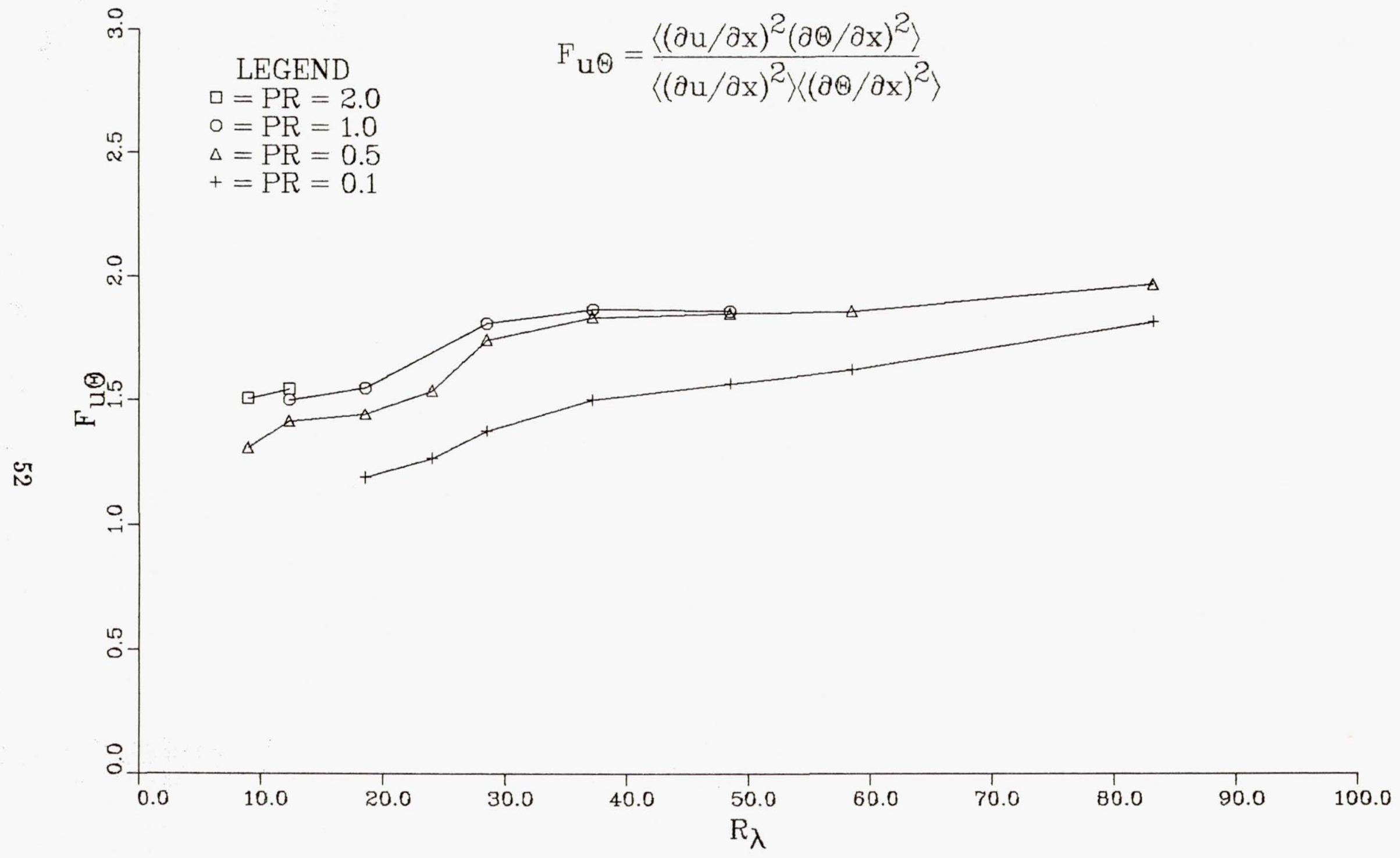

(a) Dependence of the mixed scalar-velocity derivative correlation, $F_{u \theta}(40)$, on Taylor-microscale Reynolds number.

Figure 12.- Scalar-velocity derivative correlations. 


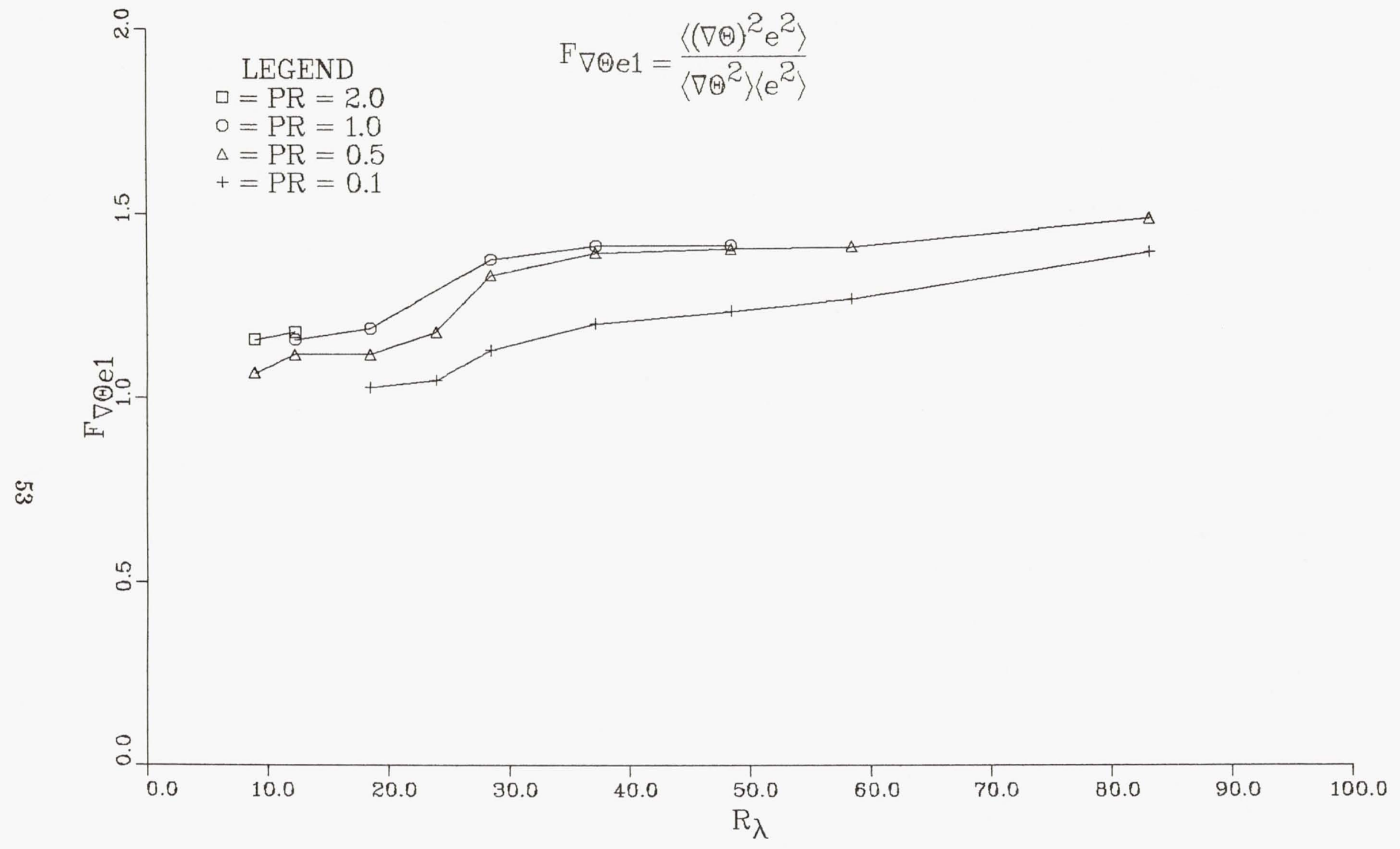

(b) Dependence of the first scalar dissipation-strain correlation, $F_{\nabla \theta e_{1}}(44)$, on Taylor-microscale Reynolds number.

Figure 12.- Continued. 


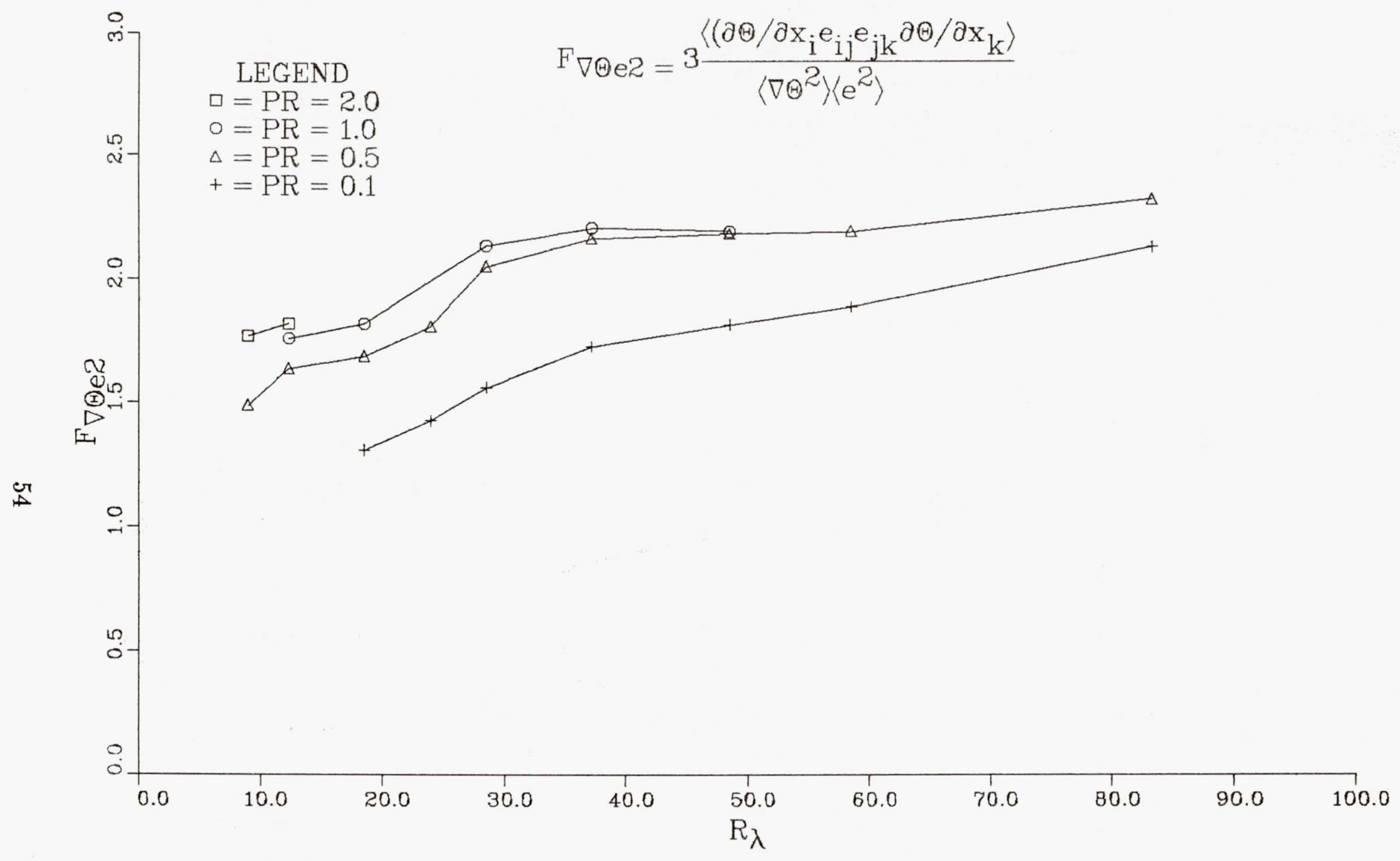

(c) Dependence of the second scalar dissipation-strain correlation, $F_{\nabla \theta e_{2}}$ (45), on Taylor-microscale Reynolds number.

Figure 12.- Concluded. 


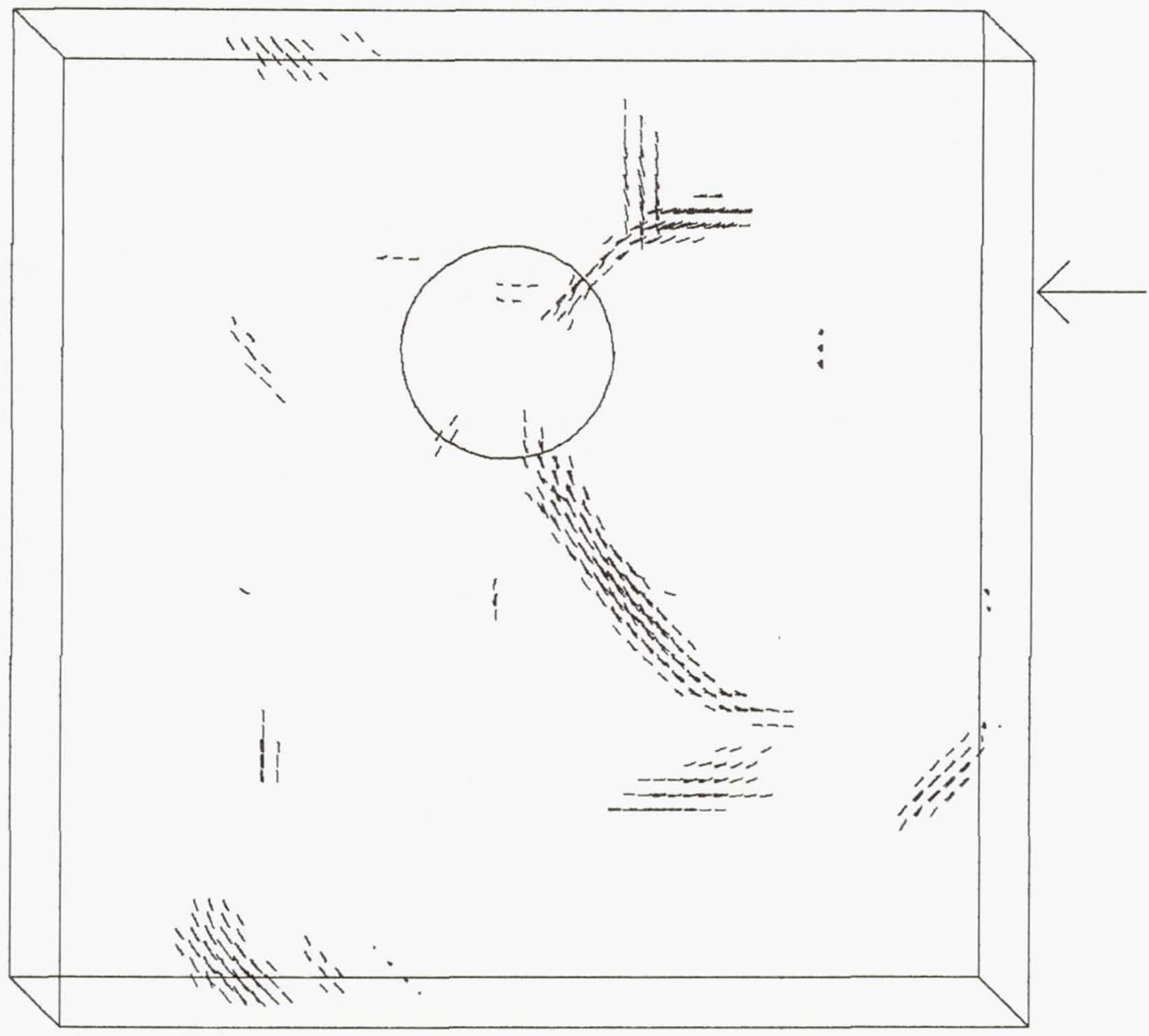

(a) About 500 lines are plotted. A strong vortex structure extends most of the way across the box. The circle indicates where the vorticity of the structure is just below the threshold. From this perspective the vortex structure appears to be a tube. The arrow indicates where a two-dimensional slice is taken (figs. 15, 16, and 18).

Figure 13.- Three-dimensional perspective plots of the vorticity field from run F19 $\left(R_{\lambda}=48.5\right)$. Short lines two mesh spacings long in the direction of the vorticity are plotted at the points of a $64^{3}$ mesh if the vorticity is above a threshold. 


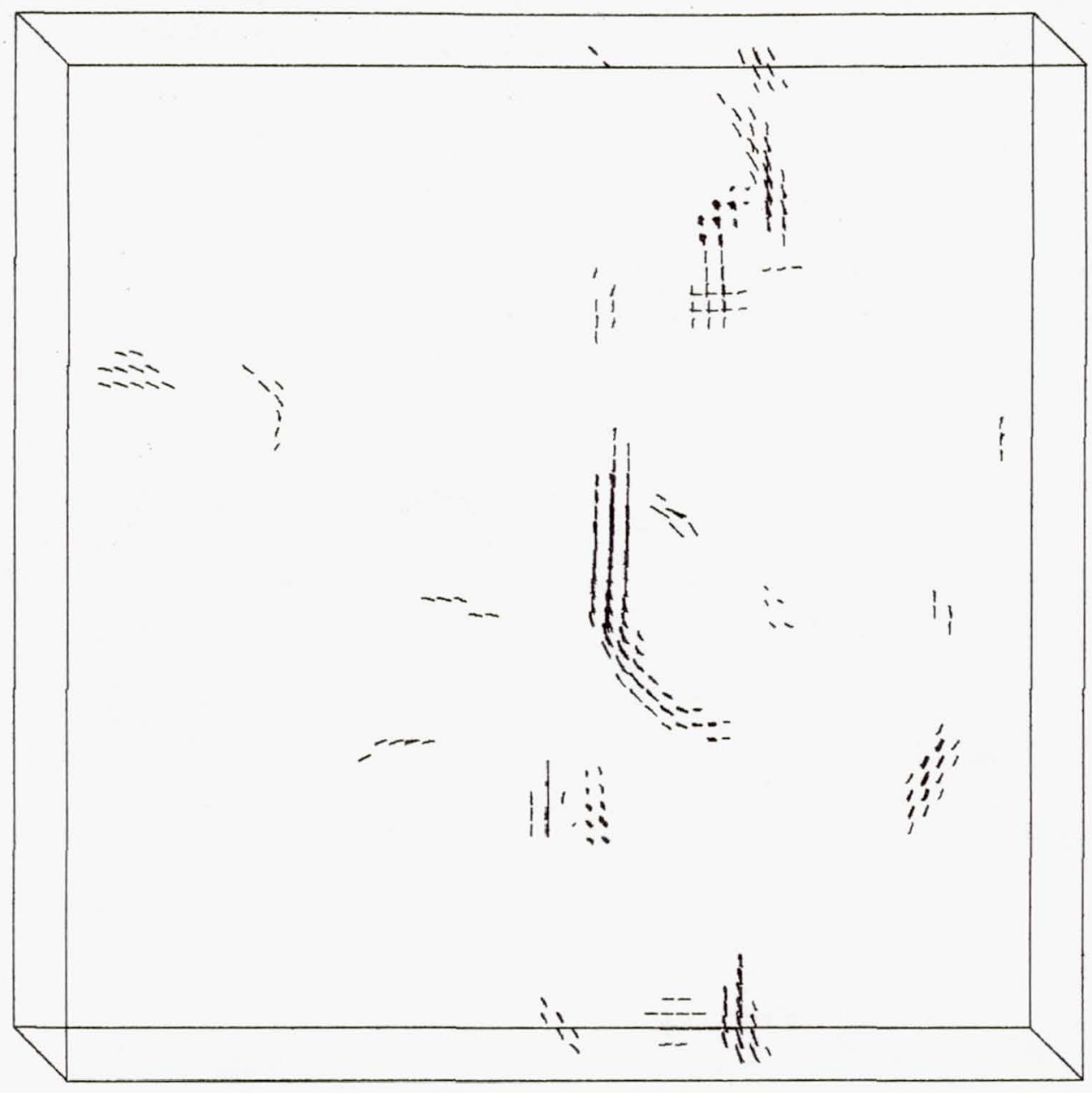

(b) Same as (a), but rotated $90^{\circ}$ about the vertical axis. In the middle the vortex structure continues to look like a tube. In the upper right it appears more twisted.

Figure 13.- Continued. 


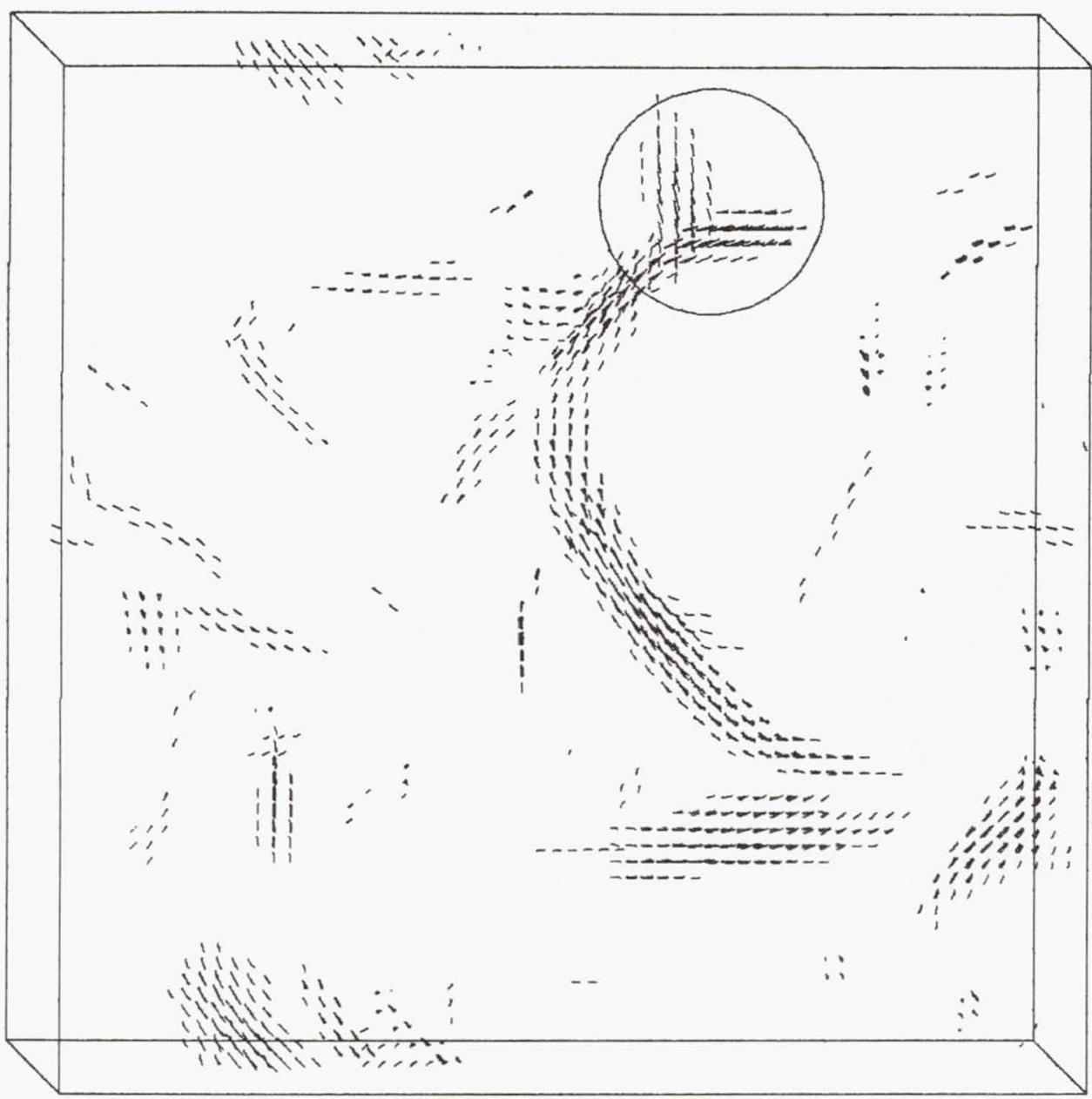

(c) Same perspective as (a), but 2000 lines are plotted to show that the vortex structure does extend across the box. The circle indicates where two vortex structures might be merging into one.

Figure 13.- Concluded. 


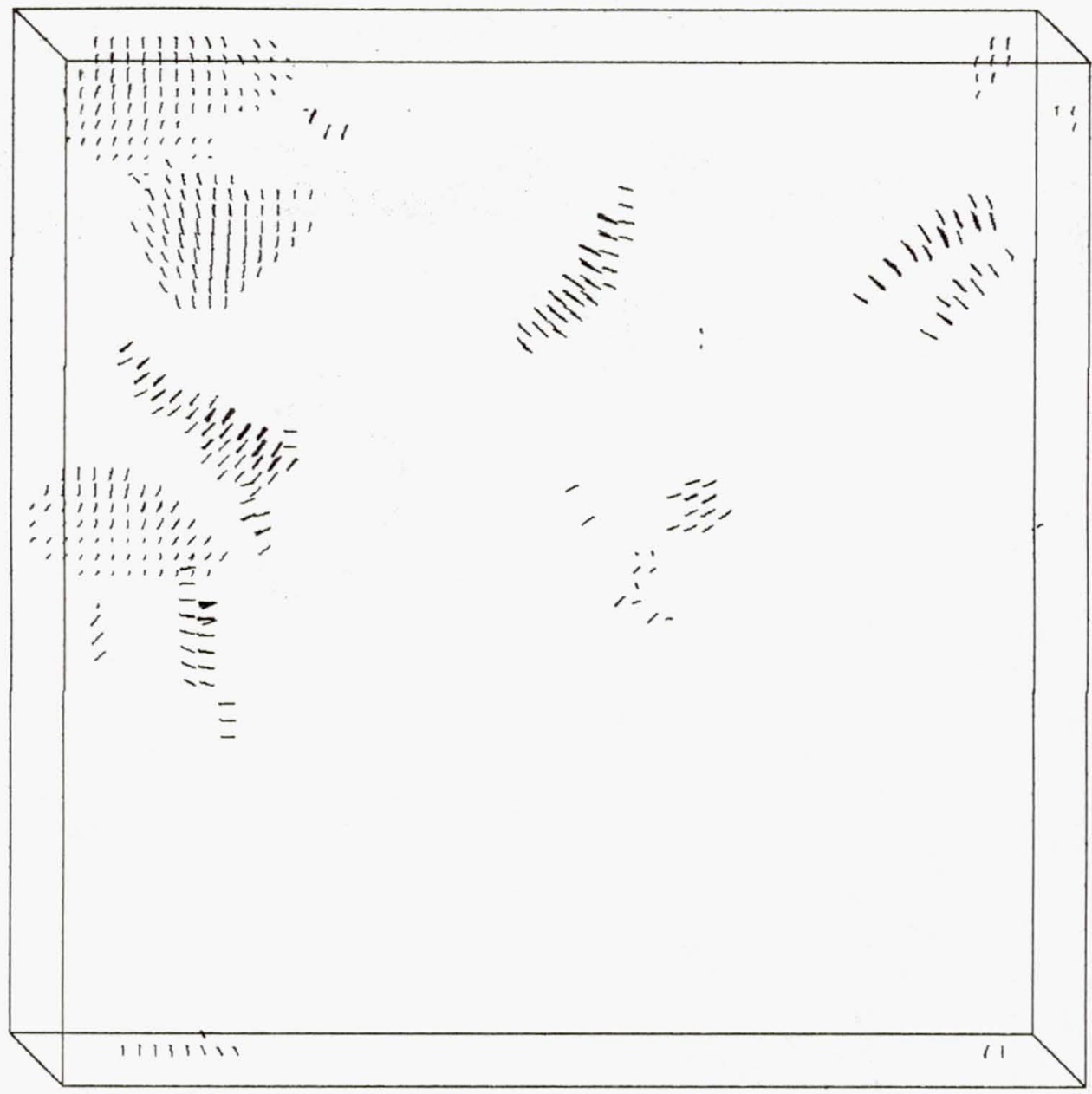

(a) The same perspective as in figure 13(a). The scalar-gradient structure in the top center is aligned along with the vortex structure in figure 13 with the scalar gradient perpendicular to the vorticity. This scalargradient structure is a sheet seen edge on. Two scalar structures in the upper-left corner are sheets seen from the top down.

Figure 14.- Three-dimensional perspective plots of the scalar-gradient field for the same time and run (F19) as figure 13. Short lines two mesh spacings long in the direction of the scalar gradient are plotted at the points of a $64^{3}$ mesh if the scalar gradient is above a threshold. 500 lines are plotted. 


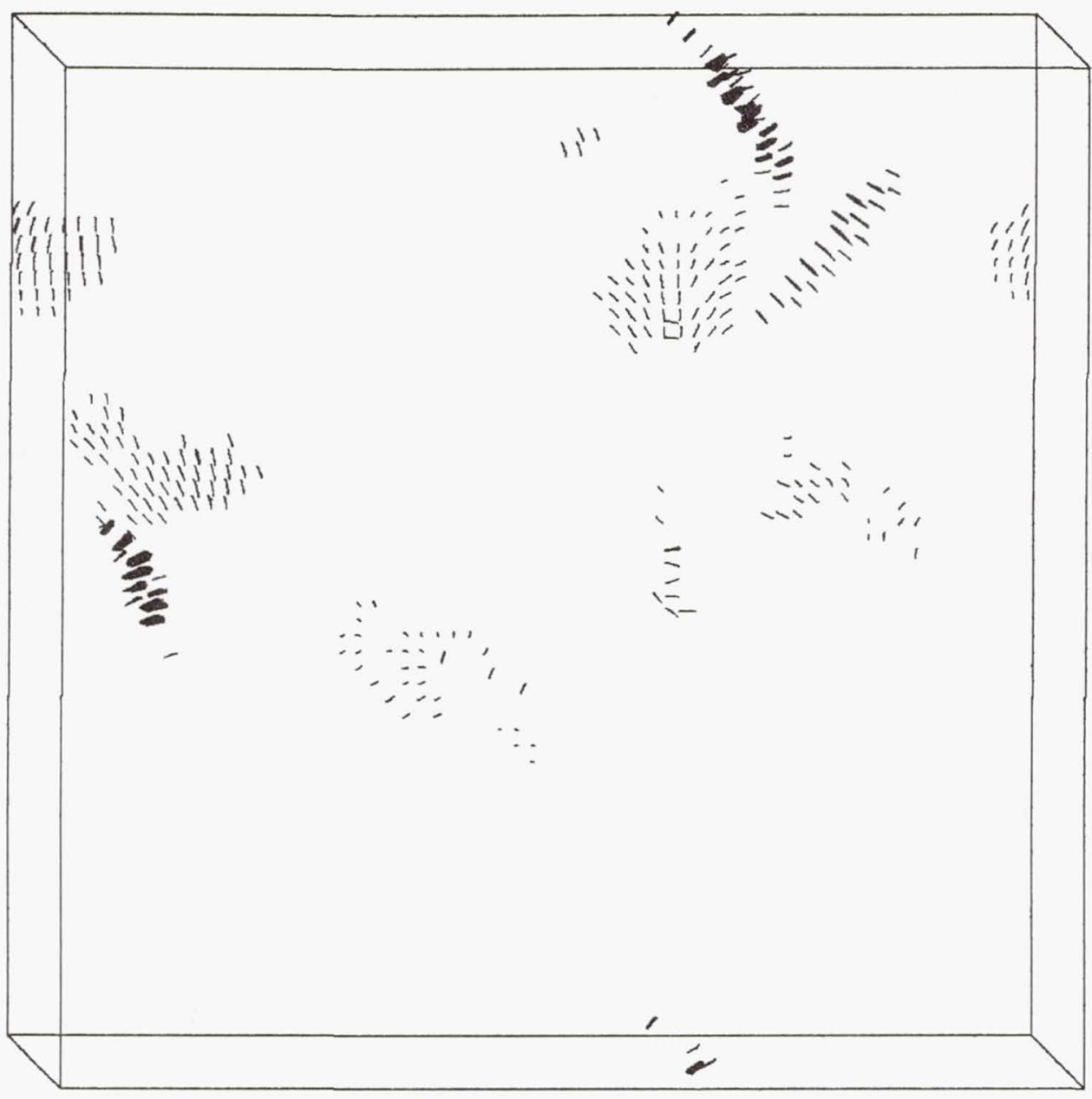

(b) The same field as (a), but rotated $90^{\circ}$ about the vertical axis. It has the same perspective as figure 13(b). The scalar-gradient structure in (a) which is aligned with the vorticity in figure 13(a) is the fan-like structure in the upper center. The structures to the right and above the fan-like structure are the sheets in the upper-left corner in (a), but now seen edge on.

Figure 14.- Concluded. 


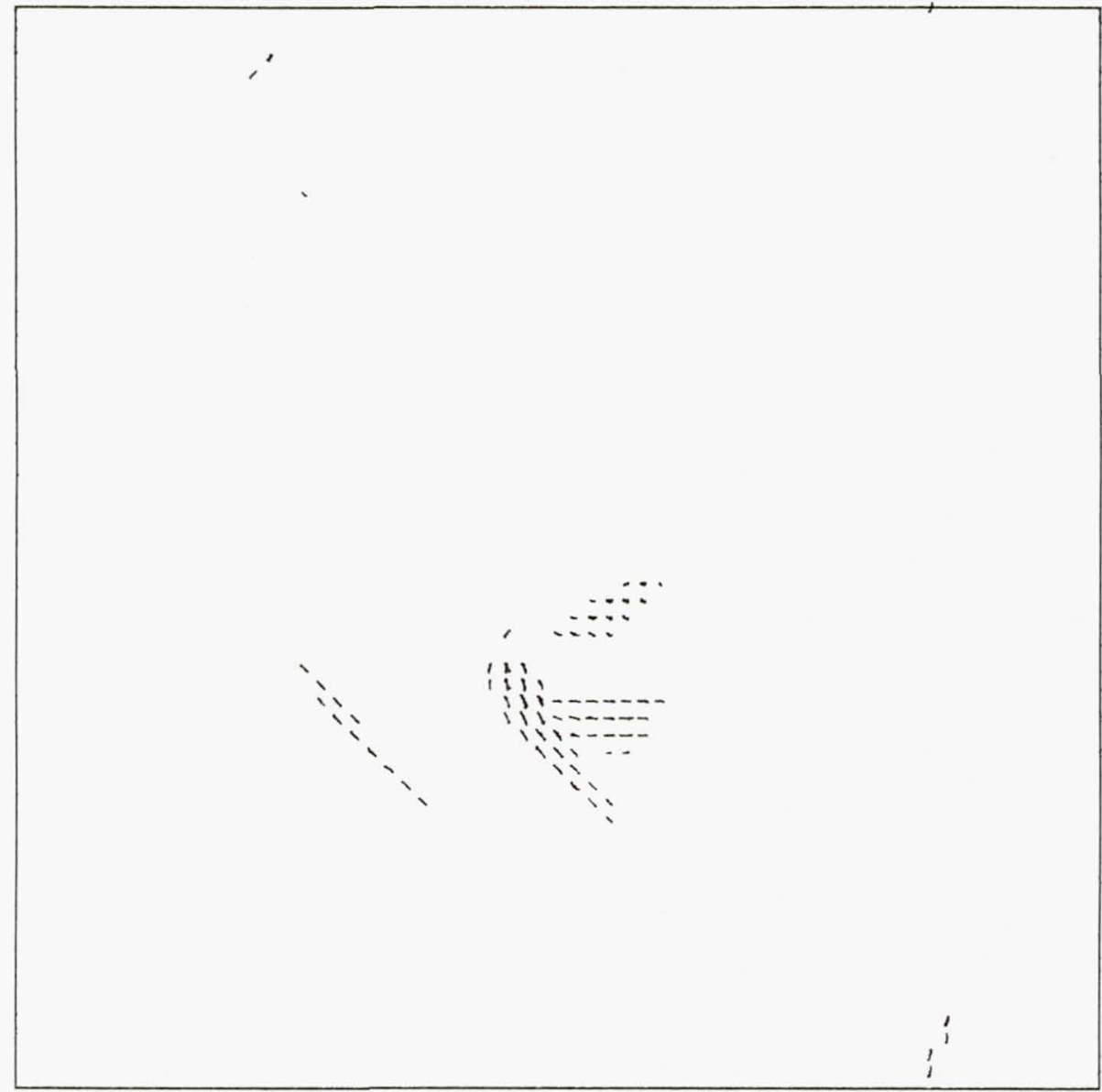

Figure 15.- Two-dimensional slice of vorticity as indicated by the arrow in figure 13(a). The structure in the center might be associated with vortex merging. 


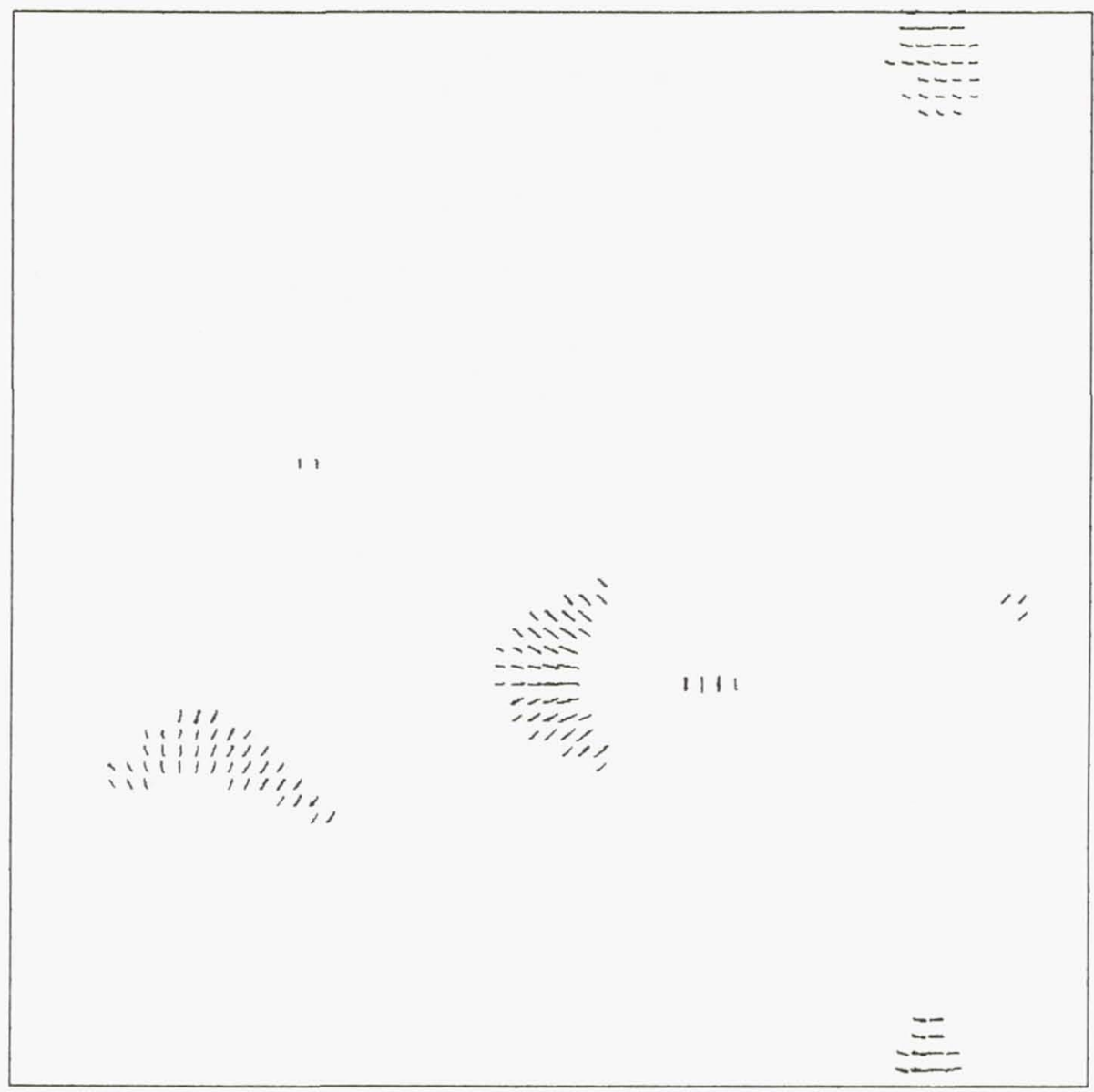

Figure 16.- Two-dimensional slice of the scalar gradient (figure 14) indicated by the arrow in figure 13(a). The structure in the center is partially wound around the vortex structure in figure 15 and shows the scalar gradient aligned perpendicular to the vorticity. 


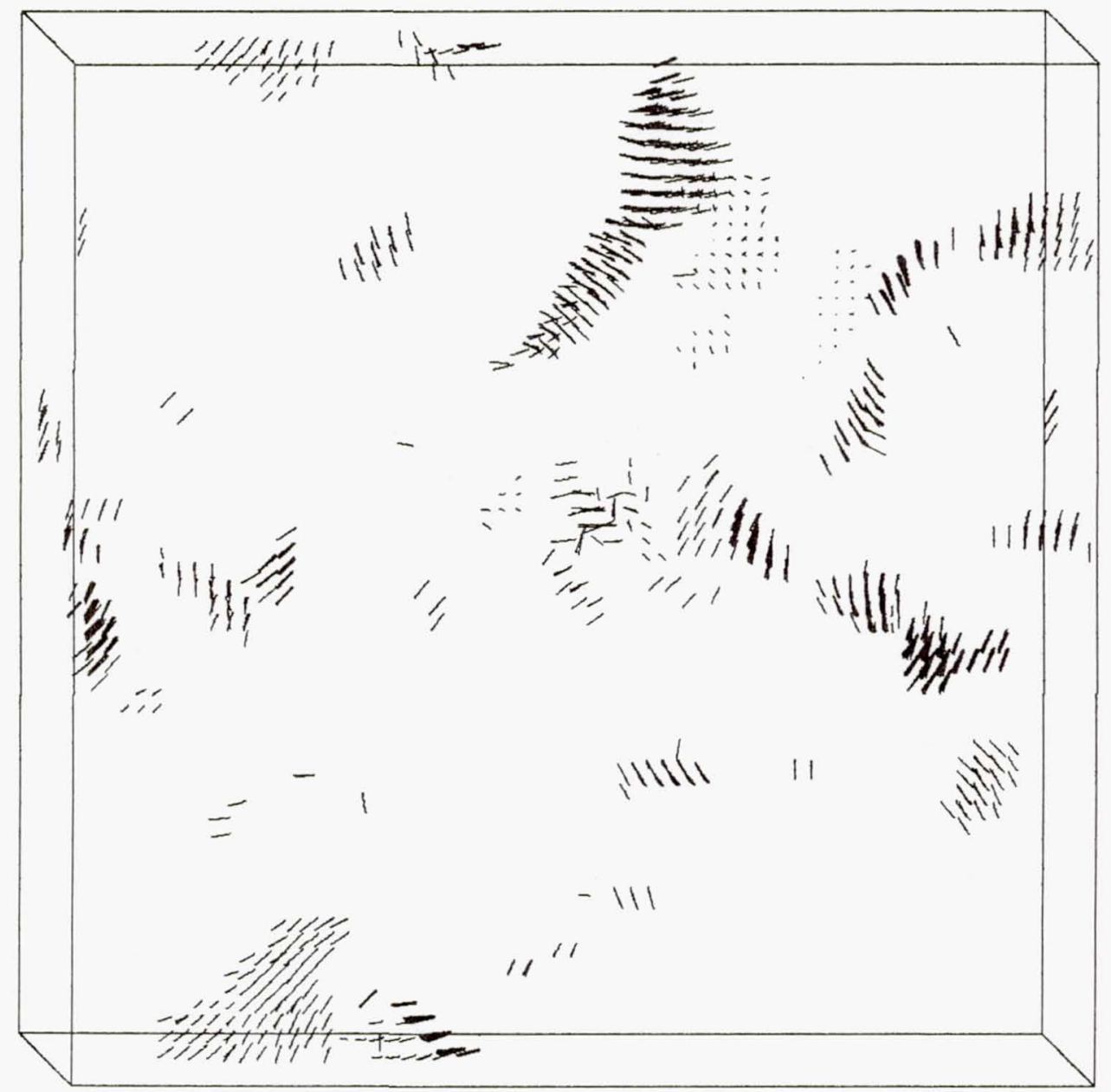

(a) The same perspective as in figures 13(a) and 14(a). The rate-of-strain structure in the top center is aligned along the vortex structure in figure 13 and the scalar-gradient structure in figure 14 with the compressive component of the rate of strain perpendicular to the vorticity and parallel to the scalar gradient.

Figure 17.- Three-dimensional perspective plots of the compressive component of the rate-of-strain field for the same time and run (F19) as figures 13 and 14. Short lines two mesh-spacings long in the direction of the compressive component are plotted at the points of a $64^{3}$ mesh if the total rate of strain is above a threshold. 1000 lines are plotted. 


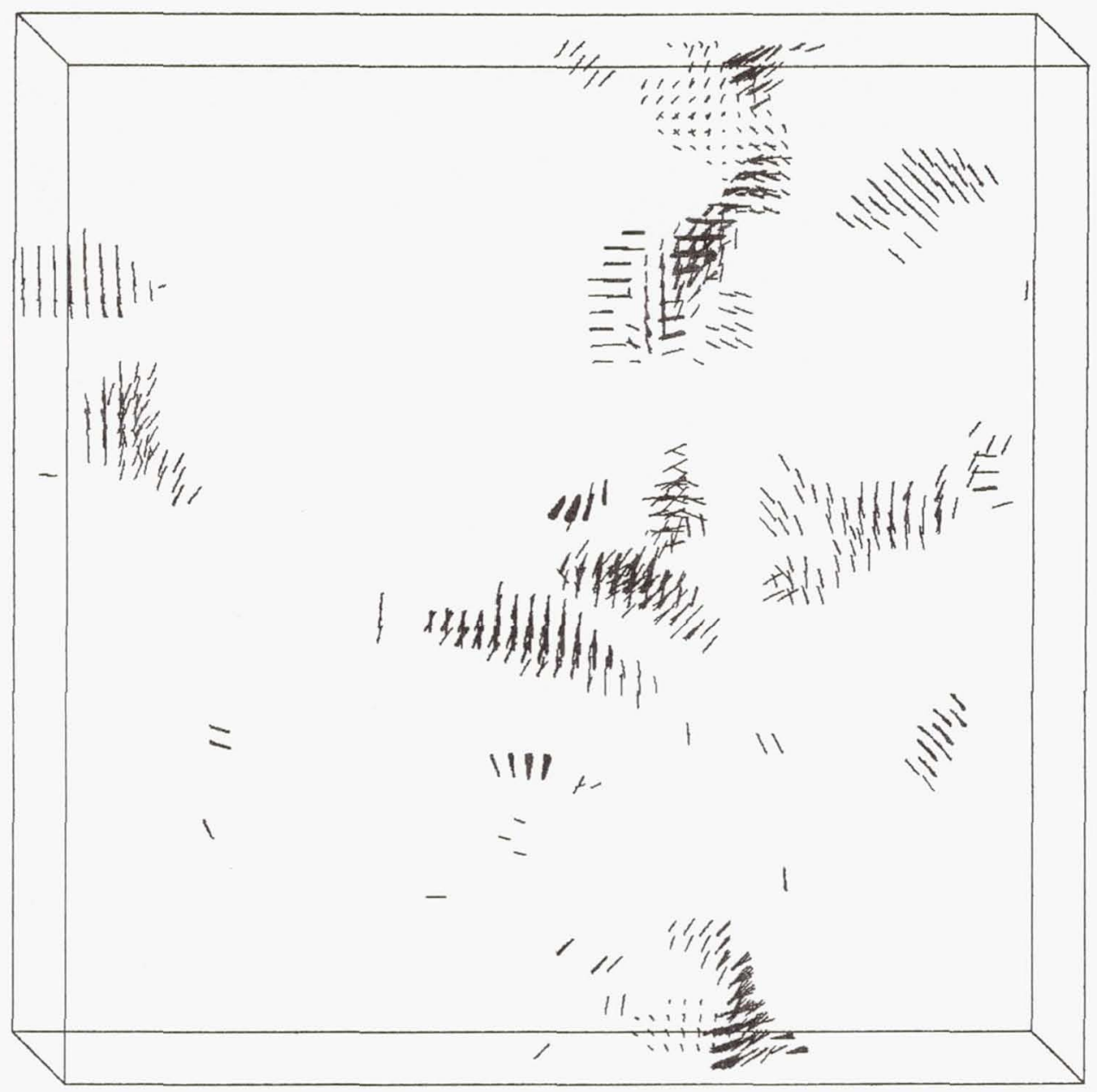

(b) The same field as (a), but rotated $90^{\circ}$ about the vertical axis. It has the same perspective as figures 13 (b) and 14(b). The rate-of-strain structure in (a) which is aligned with the vorticity and scalar gradient is the fan-like structure in the upper center.

Figure 17.- Concluded. 


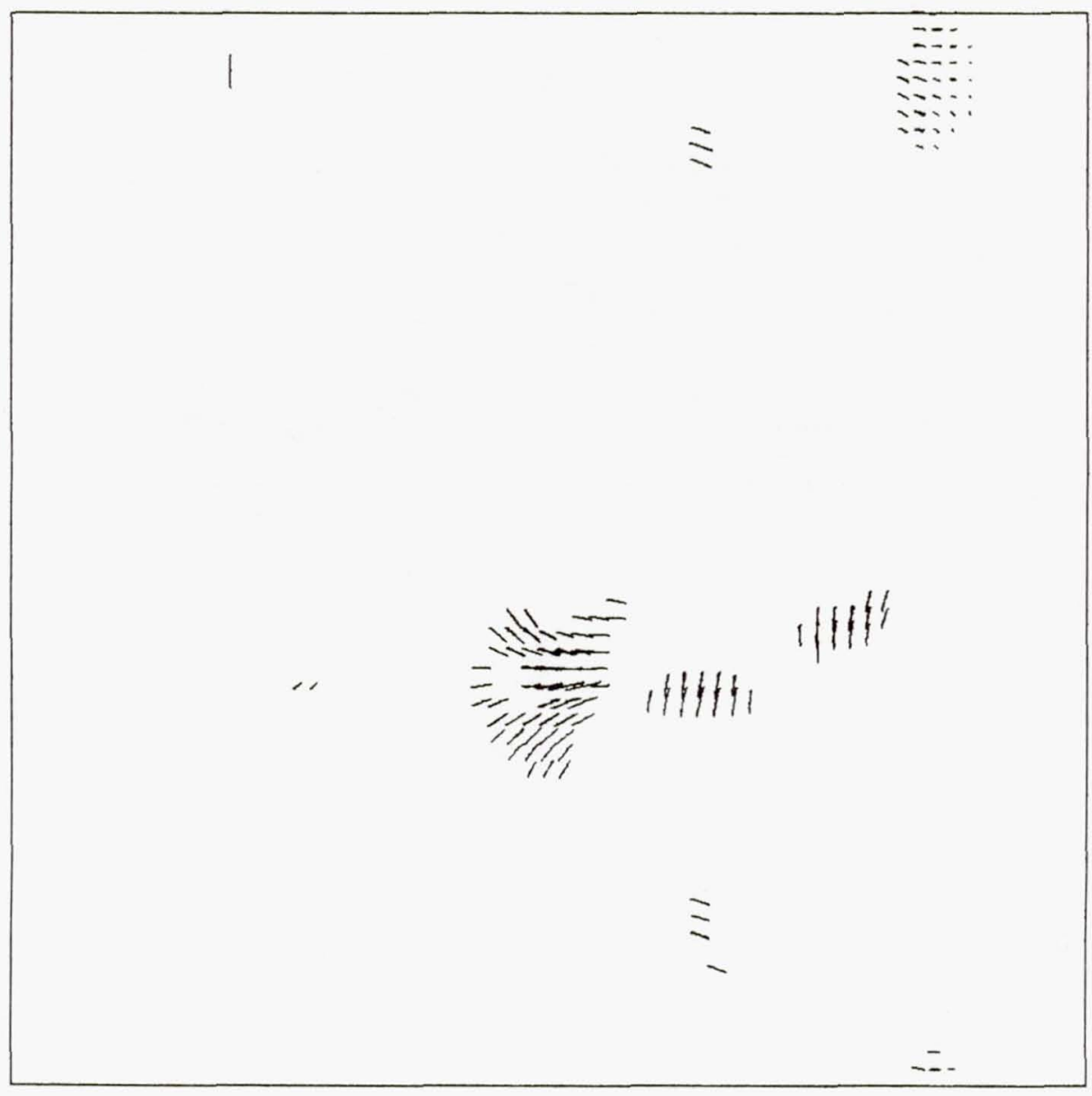

Figure 18.- Two-dimensional slice of the compressive component of the rate of strain (fig. 17) indicated by the arrow in figure 13(a). The structure in the center shows the compressive component aligned perpendicular to the vorticity in figure 15 and parallel to the scalar gradient in figure 16. 


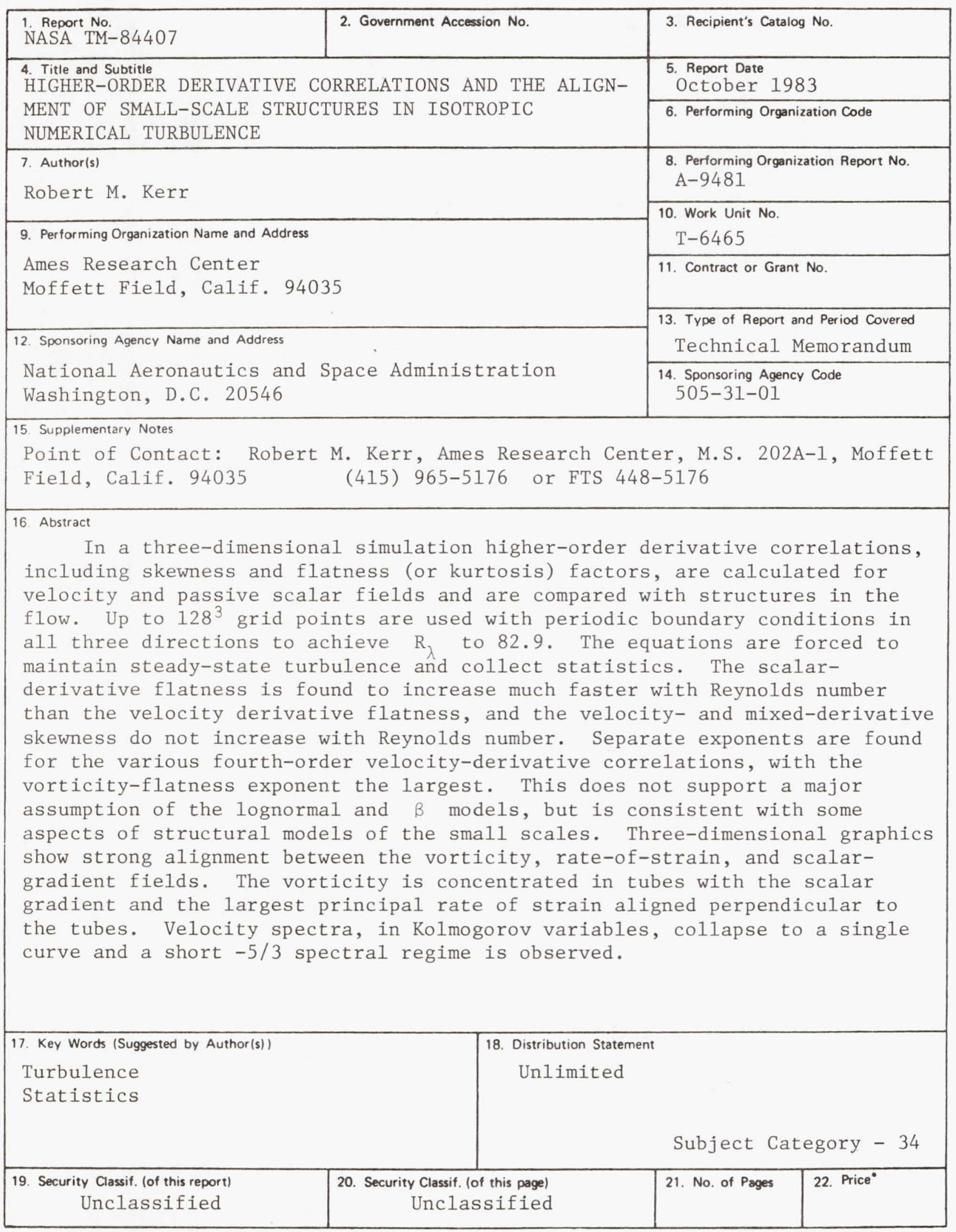

- For sale by the National Technical Information Service, Springfield, Virginia 22161 